\title{
Biodiversity Assessment of the Fishes of Saba Bank Atoll, Netherlands Antilles
}

\author{
Jeffrey T. Williams ${ }^{1 *}$, Kent E. Carpenter ${ }^{2}$, James L. Van Tassell ${ }^{3}$, Paul Hoetjes ${ }^{4}$, Wes Toller $^{5 \times a}$, Peter \\ Etnoyer $^{6 \mathrm{ab}}$, Michael Smith ${ }^{7}$
}

1 Fish Division, Department of Vertebrate Zoology, National Museum of Natural History, Smithsonian Institution, Suitland, Maryland, United States of America, 2 Department of Biological Sciences, Old Dominion University, Norfolk, Virginia, United States of America, 3 Department of Ichthyology, American Museum of Natural History, New York, New York, United States of America, 4 Department of Environment and Nature, Ministry of Public Health and Social Development, Curaçao, Netherlands Antilles, 5 Saba Conservation Foundation, Fort Bay, Saba, Netherlands Antilles, 6 Harte Research Institute, Texas A\&M University-Corpus Christi, Corpus Christi, Texas, United States of America, $\mathbf{7}$ Center for Applied Biodiversity Science, Conservation International, Arlington, Virginia, United States of America

\begin{abstract}
Biodiversity surveys were conducted on Saba Bank, Netherlands Antilles, to assess ichthyofaunal richness and to compare with published surveys of other Caribbean localities. The primary objective was to estimate the total species richness of the Saba Bank ichthyofauna. A variety of sampling techniques was utilized to survey the fish species of both the visually accessible megafauna and the camouflaged and small-sized species comprising the cryptic ichthyofauna. Based on results presented herein, the number of species known on Saba Bank is increased from 42 previously known species to 270 species. Expected species-accumulation curves demonstrate that the current estimate of species richness of fishes for Saba Bank under represents the actual richness, and our knowledge of the ichthyofauna has not plateaued. The total expected fishspecies richness may be somewhere between 320 and 411 species. The Saba Bank ichthyofaunal assemblage is compared to fish assemblages found elsewhere in the Caribbean. Despite the absence of shallow or emergent shore habitats like mangroves, Saba Bank ranks as having the eighth highest ichthyofaunal richness of surveyed localities in the Greater Caribbean. Some degree of habitat heterogeneity was evident. Fore-reef, patch-reef, and lagoonal habitats were sampled. Fish assemblages were significantly different between habitats. Species richness was highest on the fore reef, but 11 species were found only at lagoonal sites. A comprehensive, annotated list of the fishes currently known to occur on Saba Bank, Netherland Antilles, is provided and color photographs of freshly collected specimens are presented for 165 of the listed species of Saba Bank fishes to facilitate identification and taxonomic comparison with similar taxa at other localities. Coloration of some species is shown for the first time. Preliminary analysis indicates that at least six undescribed new species were collected during the survey and these are indicated in the annotated list.
\end{abstract}

Citation: Williams JT, Carpenter KE, Van Tassell JL, Hoetjes P, Toller W, et al. (2010) Biodiversity Assessment of the Fishes of Saba Bank Atoll, Netherlands Antilles. PLoS ONE 5(5): e10676. doi:10.1371/journal.pone.0010676

Editor: Brian Gratwicke, Smithsonian's National Zoological Park, United States of America

Received November 13, 2009; Accepted January 21, 2010; Published May 21, 2010

This is an open-access article distributed under the terms of the Creative Commons Public Domain declaration which stipulates that, once placed in the public domain, this work may be freely reproduced, distributed, transmitted, modified, built upon, or otherwise used by anyone for any lawful purpose.

Funding: The Department of Environment and Nature of the Netherlands Antilles and Conservation International provided funding for this project. The funders had no role in study design, data collection and analysis, decision to publish, or preparation of the manuscript.

Competing Interests: Dr. Peter Etnoyer co-authored the Deep Sea News blog with PLoS One editor Dr. Craig McClain 2005-2009. Dr. McClain has recused himself from the Saba Bank Biodiversity Assessment volume for this reason.

*E-mail: williamsjt@si.edu

aa Current address: MSC Accreditation Program, Accreditation Services International GmbH, Bonn, Germany

ab Current address: Center for Coastal Environmental Health and Biomolecular Research, National Oceanic and Atmospheric Administration, Charleston, South Carolina, United States of America

\section{Introduction}

Saba Bank is the largest atoll in the Atlantic Ocean Basin and one of the three largest atolls on earth [1]. Located in the Dutch Windward Islands about $250 \mathrm{~km}$ east of Puerto Rico, it is a flattopped seamount rising $1800 \mathrm{~m}$ from the surrounding sea floor. Except for the fact that it does not break the water surface, Saba Bank is a classic atoll consisting of a submerged mountain crowned at the summit with a ring of actively growing coral reefs [2]. Saba Bank is relatively free of the problems that are degrading many Caribbean reef systems, and the few problems it faces include anchoring and abrasion by oil tankers maneuvering off the petroleum transshipment facilities on St. Eustatius, potential petroleum spillage and subsequent use of dispersants, general vessel passage in a zone of high maritime traffic, possible overfishing for certain species, and exploration for petroleum reserves (so far unsuccessful). Saba Bank's fisheries and dive operations are economically significant to the small community on Saba Island (about 1500 residents) that has direct responsibility for its management.

The known fish fauna of Saba Bank prior to our survey consisted of 42 fish species. Most of these species were taken during fishery bottom-trawl surveys on Saba Bank, including two M/V Oregon stations in 1958 and nine stations in 1959, and two trawl hauls taken in 1969 by the R/V Pillsbury. Although four of these trawls were taken on or near the top of Saba Bank, nine were on the deep outer slopes. The habitats sampled during these surveys were restricted to relatively soft-bottom habitats due to the exclusive use of trawling techniques. These trawl samples provided valuable records of fishes living on soft bottoms and on the outer slopes of Saba Bank. 
A biodiversity-assessment survey was carried out on Saba Bank during 2006 and 2007, with a major goal being to improve knowledge of the biodiversity on one of the world's most significant, though poorly known, coral-capped seamounts. In an effort to record as many fish species as possible in the short period of time available for the survey, we utilized a variety of fish sampling techniques. These techniques included visual surveys by divers, use of SCUBA to apply ichthyocide (a natural fish toxicant consisting of dried and powdered Derris root - assayed at 7.5\% rotenone), hand-line fishing, by-catch from lobster and fish traps taken by local fishermen, and port sampling observations of fish landings. During the surveys, an attempt was made to obtain a photograph documenting the fresh colors of as many species as possible, a tissue sample of each species, and preserved specimen vouchers that have been archived in the National fish collection (USNM) of the National Museum of Natural History (NMNH), Smithsonian Institution (SI). Specimens representing six or more undescribed species and two rare gobies, Pycnomma roosevelti and Psilotris boehlkei, were collected.

The habitats surveyed on the Saba Bank "atoll" during this study are classified as: fore reef, patch reef, lagoonal and Small Bank. Fore-reef areas are located around the outer rim of the submerged atoll. Patch reef is an isolated portion of "reef" situated on the lagoonal (interior) side of the fore reef. Lagoonal is the central portion of the atoll interior to the fore-reefs around the atoll rim and may have a variety of bottom types. Small Bank is a small, independent "seamount-like" structure located off the northwest corner of Saba Bank with its shallowest depth at about $11 \mathrm{~m}$. Depth categories were arbitrarily assigned as shallow (11$24 \mathrm{~m})$, mid-depth (25-34 m), and deep (35-38 m).

The primary goals of the overall biodiversity survey were to provide data and analysis to support designation of Saba Bank as a marine protected area, to support the development of a management plan, and to contribute to a petition to the International Maritime Organization to designate appropriate parts of Saba Bank as a Particularly Sensitive Sea Area.

\section{Results}

We occupied fish stations at 25 locations during a rapid assessment (RAP) survey, 2-16 January 2006; including: 20 ichthyocide stations, 12 roving visual surveys, two hook \& line stations, and five by-catch stations from lobster traps; two fishichthyocide stations were occupied at two additional locations on 20 June 2007 (Figure 1). In 2007, Toller assessed the benthic communities and fish assemblages based in part on 40 visual surveys of the fish fauna at an area on the eastern side of Saba Bank.

Specimens collected during the RAP survey were preserved as vouchers and processed into the fish collection (available online at http://vertebrates.si.edu/fishes/fishes_collections.html) at the NMNH (as many species as possible were also tissue sampled

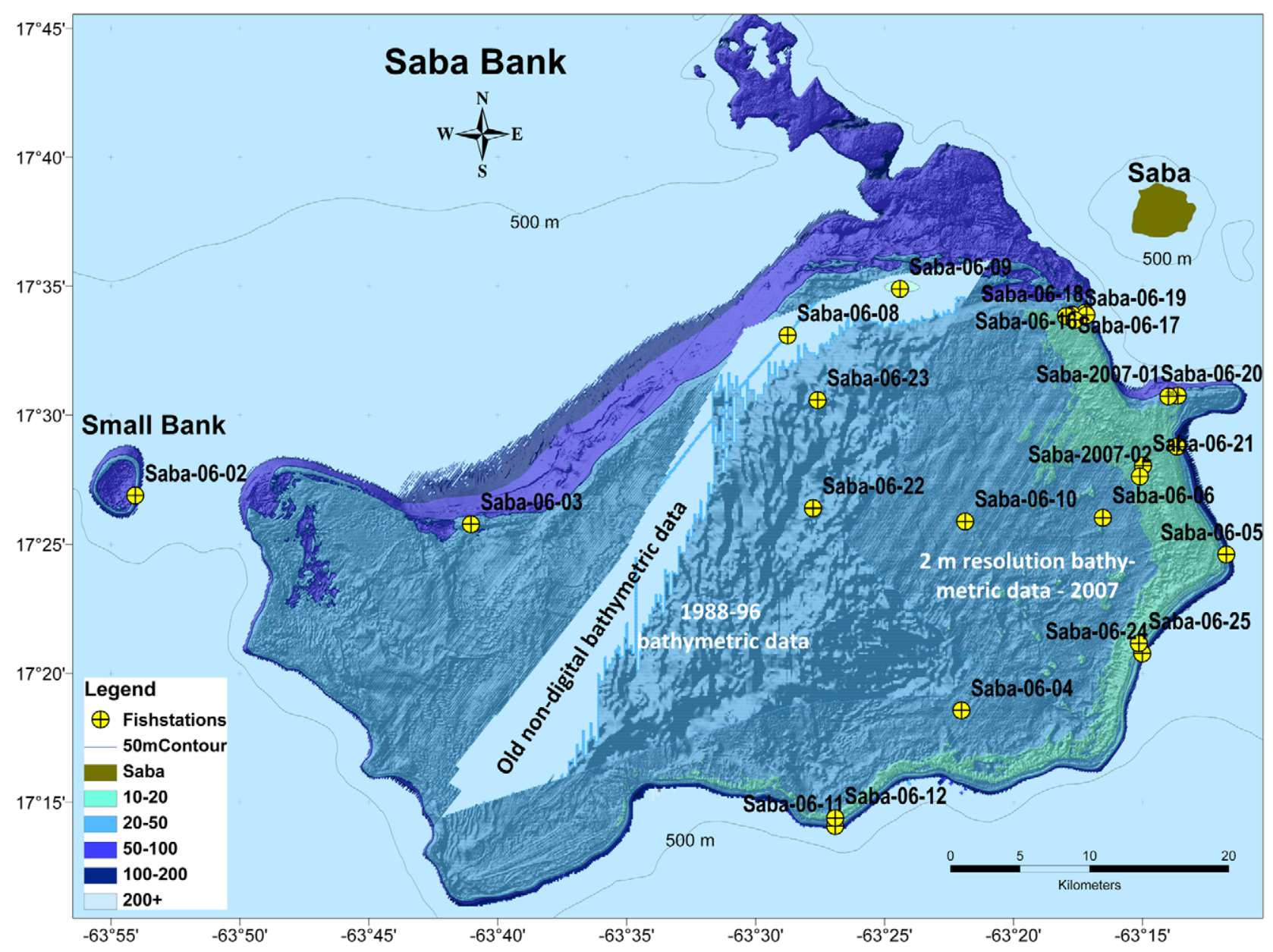

Figure 1. Bathymetric map of Saba Bank with fish stations marked. doi:10.1371/journal.pone.0010676.g001 
and photographed in the field). Toller collected and preserved vouchers from fishery landings when possible and photographed specimens representing new records. Toller's vouchers were processed into the fish collection at the $\mathrm{NMNH}$ and are also available in the online database.

Some species were only taken during fishery bottom trawl surveys by the M/V Oregon $(1958,1959)$ and the R/V Pillsbury (1969) on Saba Bank. Species represented by voucher specimens in museum collections are included in our comprehensive species list.

A non-metric multidimensional-scaling (MDS) ordination of the 12 rotenone and roving stations based on a Bray-Curtis similarity matrix of incidence data was used to illustrate similarities and differences of fish assemblages found on the four atoll habitat types: fore reef, patch reef, lagoonal, Small Bank (Figure 2). There were significant differences among habitat types (ANOSIM Global $\mathrm{R}=0.96, P=0.001)$. Differences between fore-reef stations and patch-reef stations were most pronounced (ANOSIM, Pairwise $\mathrm{R}=0.92, P=0.002$ ). Fore-reef assemblages ranged from 39 to 60 species per station while the patch-reef stations had 26 and 32 species. Fore-reef stations were $50 \%$ similar to each other, and patch-reef stations were up to $60 \%$ similar. Fore-reef assemblages were not significantly different than Small Bank, or the lagoonal habitat (ANOSIM, Pairwise $\mathrm{R}=0.973, \mathrm{P}=0.11$ ). Low sample size (one station with 39 species) in the lagoonal habitat limits this comparison.

There were significant differences in the fish assemblages when classified by depth - shallow, middle, and deep (ANOSIM Global test, $\mathrm{R}=0.618, \mathrm{P}=0.006)$. Differences in species composition were most evident between mid-depth and deep sites (ANOSIM Pairwise test, $\mathrm{R}=0.829, \mathrm{P}=0.008$ ). Fore-reef sites were typically at mid to shallow depths (20 to $34 \mathrm{~m}$ ) whereas the patch-reef sites were typically deep (35 to $38 \mathrm{~m}$ ). There is some evidence of habitat heterogeneity and vertical zonation for fish assemblages on Saba Bank, but more sampling is necessary to discern whether habitat or depth best explains the differences among groups.

The horizontal axis in the MDS plot represents a gradient in species richness with patch-reef and lagoonal sites having lowest richness ( Figure 2). The vertical axis in the MDS plot illustrates differences related to depth with deepest station (Small Bank) higher in the vertical axis, and shallower sites lower in the vertical axis. The two-dimensional stress value was low in the MDS (stress $=0.1)$ indicating a slight chance of misrepresentation.

A principal components analysis (PCA) was used to understand which species assemblages were responsible for differences among stations. The first three components explained $43.9 \%$ of the variation. The first component (18.6\% of variation explained) was dominated by ubiquitous species and most common species. Strongest loadings (negative) on the first principal component (PC1) included the four species found at all stations (Halichoeres garnoti, Serranus tigrinus, Stegastes partitus, and Thalassoma bifasciatum) and 13 species found at all but one to four of the stations. These 13 common species are rarely found at the small bank, patch-reef, and lagoonal stations and, therefore, PC1 also serves to define the fore-reef sites. For example, Acanthurus bahianus, Coryphopterus glaucofraenum, Coryphopterus dicrus, and Scarus taeniopterus were found at all fore-reef sites but rarely at the Small Bank, lagoonal and at least one of the patch-reef sites. The strongest positive loadings on PC1 are from 11 species only found at the lagoonal site. Principal components 2 and 3 correspond strongly to the horizontal or species richness component on the MDS with strongest positive

\section{MDS Plot of Saba Bank Fish Surveys}

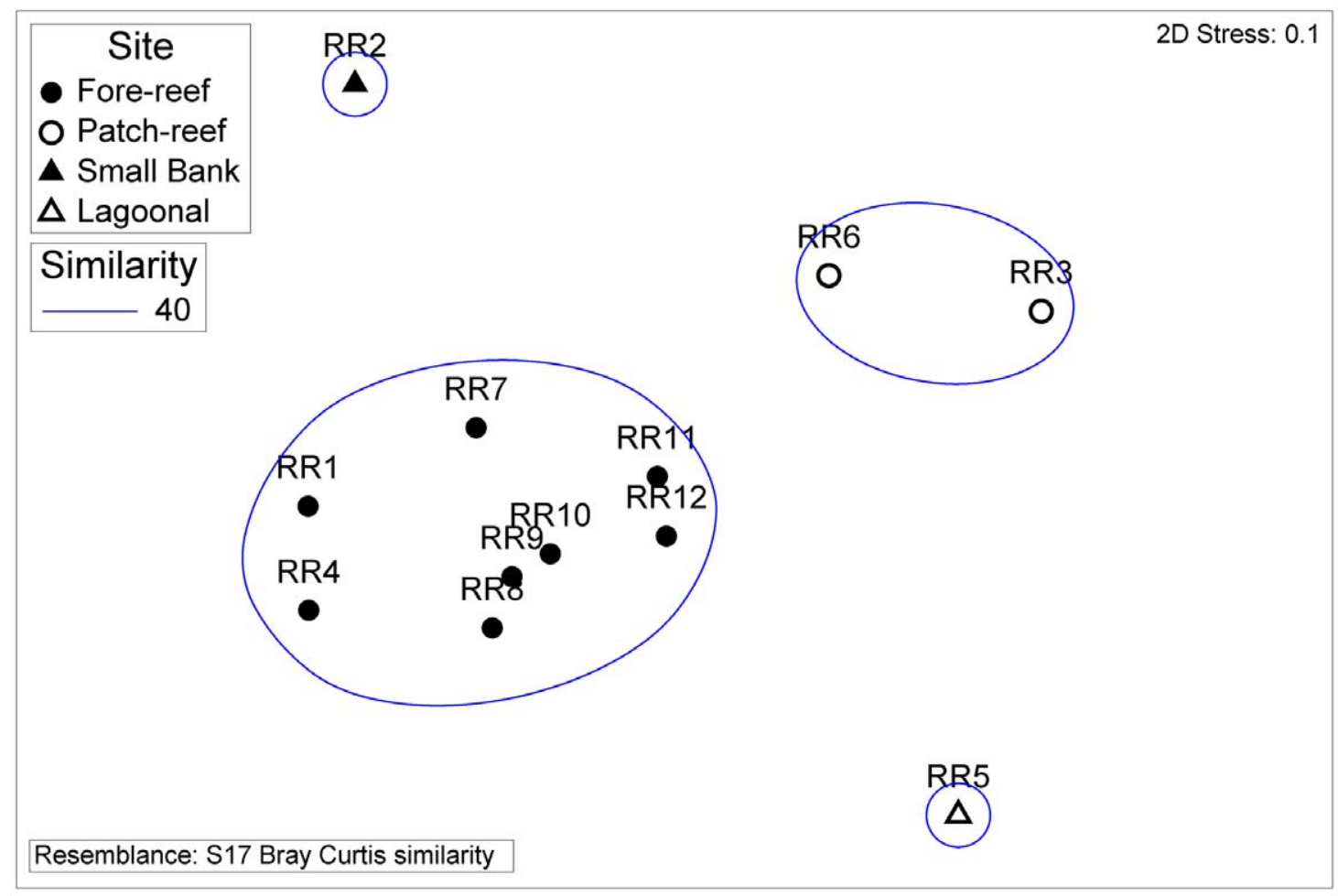

Figure 2. MDS ordination of fish-survey stations illustrates high similarity of assemblages within fore-reef sites and significant differences (ANOSIM, $P<0.05$ ) among habitats.

doi:10.1371/journal.pone.0010676.g002 
loadings on species typically found at the low to medium species richness stations 3, 5, 6 and 8-12 (e.g. Cryptotomus roseus, Haemulon melanurum, Halichoeres bivittatus, Astrapogon puncticulatus, and Serranus baldwini). Strongest negative scores were on species that were frequently found at high to medium species richness stations 1, 2, 4, 7 and 8 (e.g. Hypoplectrus puella, Lythrypnus elasson, Prognathodes aculeatus, Neoniphon marianus, and Gramma loreto).

An annotated list of the fishes of Saba Bank is provided below. In the list, we include the family, genus and species, author and English common name (as common names are not standardized internationally, we strove to apply the most widely used English common name based on FishBase (http://www.fishbase.org) listings). The use of "cf" before a species name indicates that the specimen photographed is similar to that species, but probably represents an undescribed species. Voucher specimens are archived at the National Museum of Natural History (USNM) and the Florida Museum of Natural History (UF) and each species with vouchers is annotated with the museum's acronym where the specimens are housed. The basis of each species record is indicated by: I - ichthyocide station, F - caught by a local fisherman and photographed, $\mathrm{T}$ - bottom trawl, $\mathrm{O}$ - visual sighting during Toller survey, $\mathrm{V}$ - visual sighting during RAP survey at roving and rotenone station. Lengths of specimens are recorded in $\mathrm{mm}$ for either standard length (SL), total length (TL), or fork length (FL). Photographs showing the color pattern of freshly collected specimens are included for as many of the species as possible. Images illustrating observed sexual and developmental (juvenile to adult) variability in color pattern are included where possible.

\section{Ginglymostomatidae-nurse sharks}

Ginglymostoma cirratum (Bonnaterre, 1788)—nurse shark; OV; Figure 3

\section{Squalidae-dogfish sharks}

Squalus cubensis Howell Rivero, 1936-Cuban dogfish; F; Figure 4

\section{Carcharhinidae-requiem sharks}

Carcharhinus perezii (Poey, 1876) - reef shark; F

Galeocerdo cuvier (Péron \& Lesueur, 1822) - tiger shark; F,O

\section{Etmopteridae-lantern sharks}

Etmopterus bullisi Bigelow \& Schroeder, 1957-lined lantern shark; USNM, T

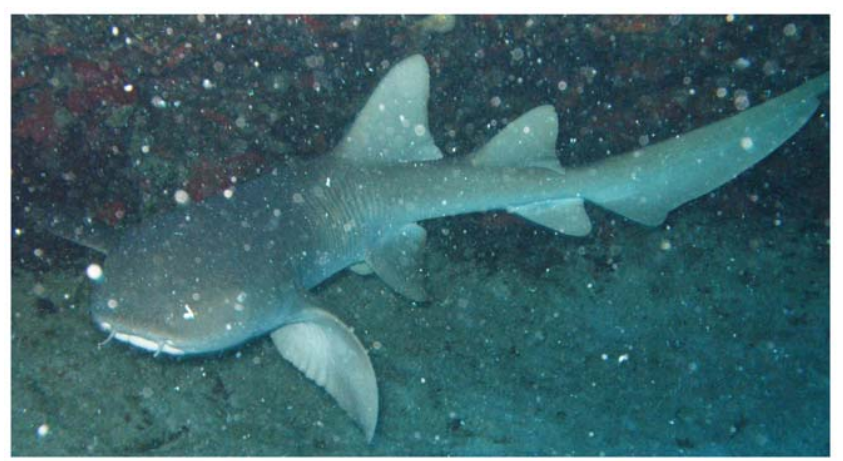

Figure 3. Ginglymostoma cirratum, underwater photo by Juan Sanchez.

doi:10.1371/journal.pone.0010676.g003

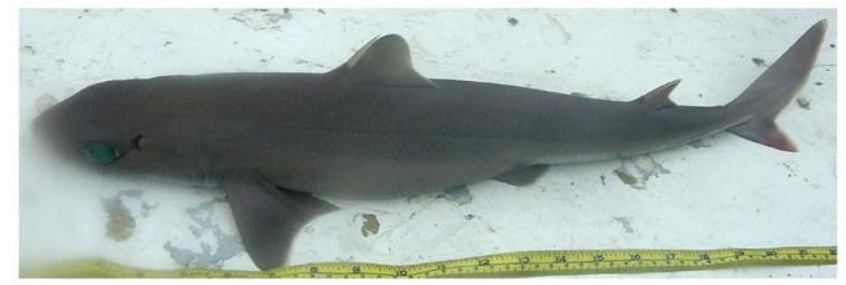

Figure 4. Squalus cubensis, $475 \mathrm{~mm}$ TL, photo by W Toller. doi:10.1371/journal.pone.0010676.g004

\section{Dasyatidae-whiptail stingrays}

Dasyatis americana Hildebrand \& Schroeder, 1928 - southern stingray; $\mathbf{V}$

\section{Muraenidae-morays}

Anarchias similis (Lea, 1913) - pygmy moray; USNM, I

Enchelycore carychroa (Böhlke \& Böhlke, 1976) — chestnut moray;

USNM, I, F; Figure 5

Enchelycore nigricans (Bonnaterre, 1788) — viper moray; USNM, I Gymnothorax conspersus Poey, 1867 - saddled moray; USNM, F;

Figure 6

Gymnothorax maderensis(Johnson, 1862)—sharktooth moray;

USNM, F; Figure 7

Gymnothorax miliaris (Kaup, 1856) - goldentail moray; USNM, I,

O; Figure 8

Gymnothorax moringa (Cuvier, 1829) — spotted moray; USNM, I,

$\mathbf{O}, \mathbf{V}$; Figure 9

Gymnothorax polygonius Poey, 1876 - polygon moray; USNM, F;

Figure 10

Gymnothorax vicinus (Castelnau, 1855)-purplemouth moray;

USNM, I, O; Figure 11

Monopenchelys acuta (Parr, 1930)-redface moray; USNM, I; Figure 12

Uropterygius macularius (Lesueur, 1825)-marbled moray; USNM, I

Ophichthidae-snake eels

Ahlia egmontis (Jordan, 1884)- key worm eel; USNM, I; Figures 13, 14

Aprognathodon platyventris Böhlke, 1967 - stripe eel; USNM, I; Figures 15, 16

Myrichthys breviceps (Richardson, 1848) — sharptail eel; $\mathbf{O}$

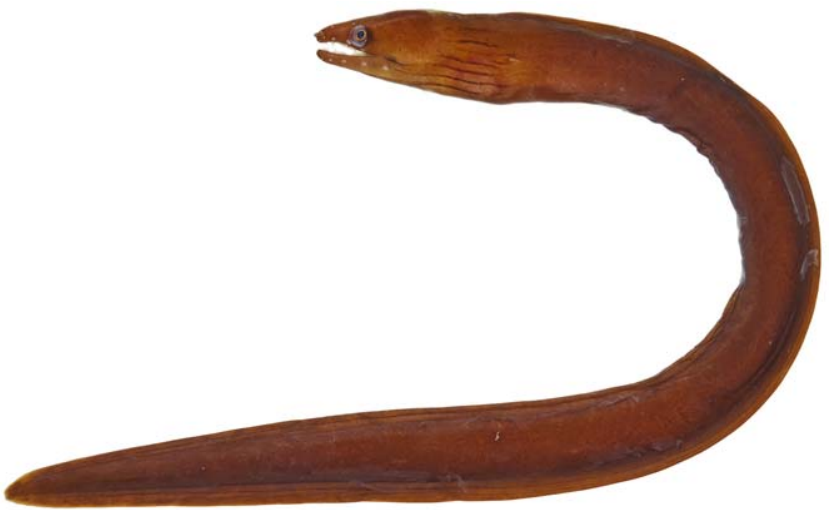

Figure 5. Enchelychore carychroa, $175 \mathrm{~mm} \mathrm{TL}$, photo by JT Williams.

doi:10.1371/journal.pone.0010676.g005 


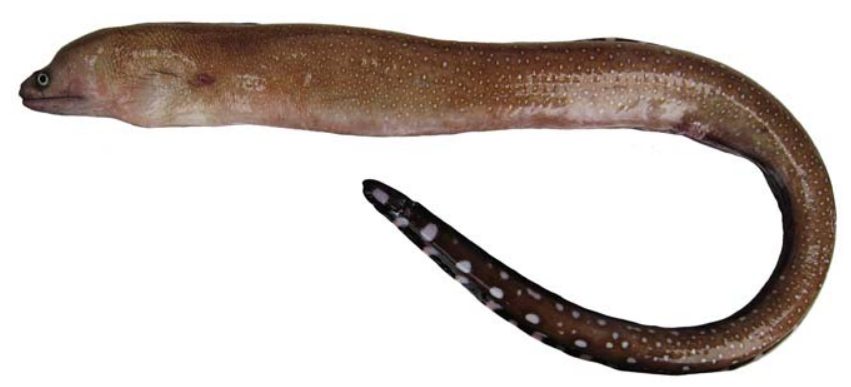

Figure 6. Gymnothorax conspersus, $730 \mathrm{~mm} \mathrm{TL}$, photo by $\mathrm{W}$ Toller.

doi:10.1371/journal.pone.0010676.g006

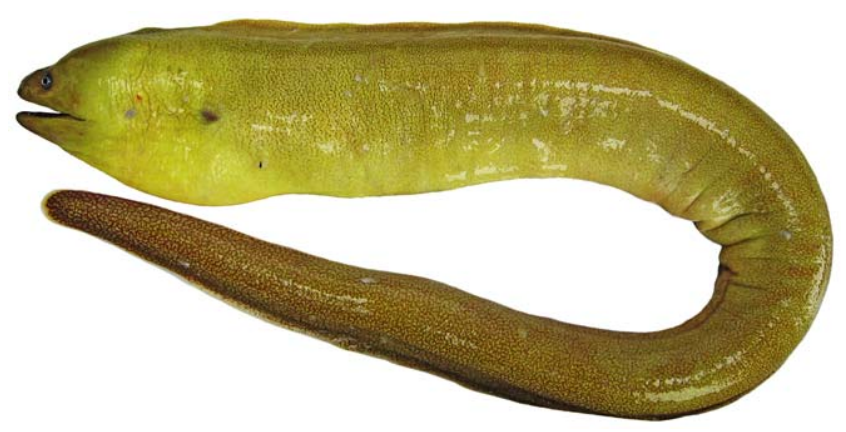

Figure 7. Gymnothorax maderensis, $300 \mathrm{~mm} \mathrm{TL}$, photo by W Toller.

doi:10.1371/journal.pone.0010676.g007

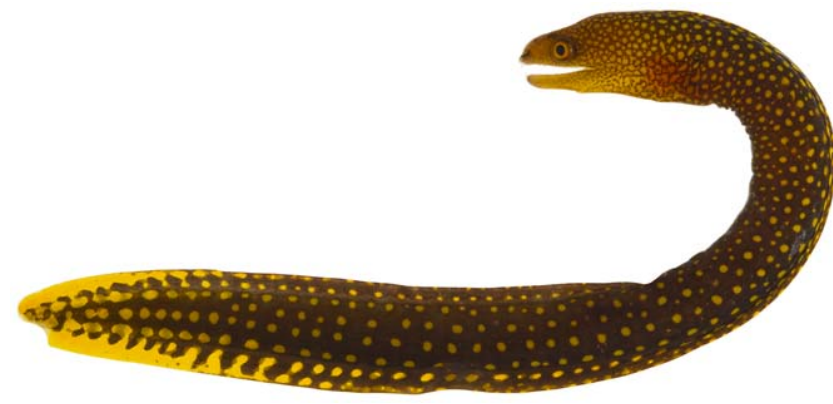

Figure 8. Gymnothorax miliaris, $60.3 \mathrm{~mm} \mathrm{TL}$, photo by JT Williams.

doi:10.1371/journal.pone.0010676.g008

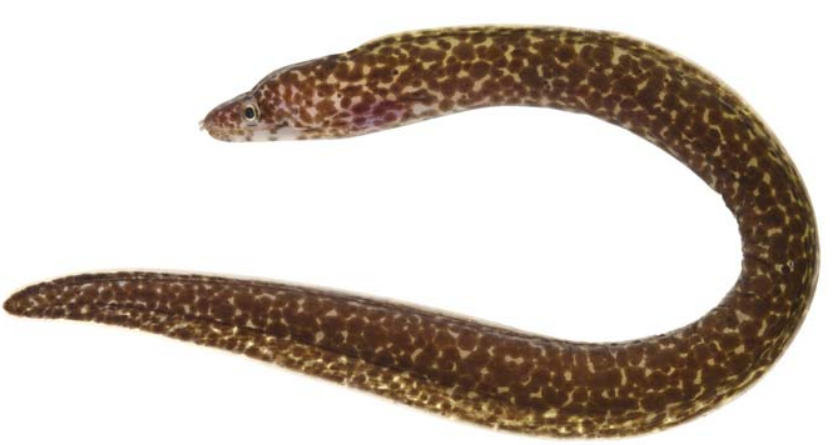

Figure 9. Gymnothorax moringa, $189.8 \mathrm{~mm} \mathrm{TL}$, photo by JT Williams.

doi:10.1371/journal.pone.0010676.g009

PLOS ONE I www.plosone.org

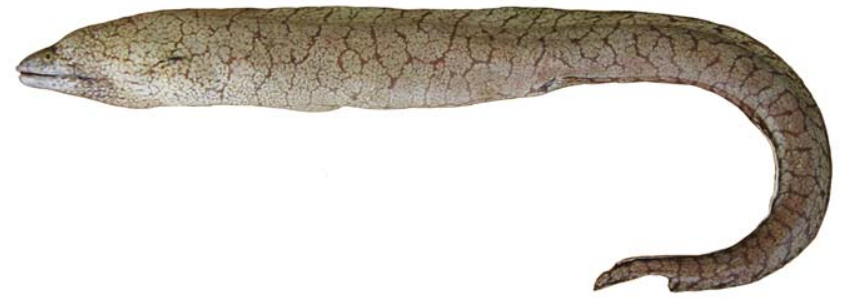

Figure 10. Gymnothorax polygonius, $810 \mathrm{~mm} \mathrm{TL}$, photo by W Toller.

doi:10.1371/journal.pone.0010676.g010

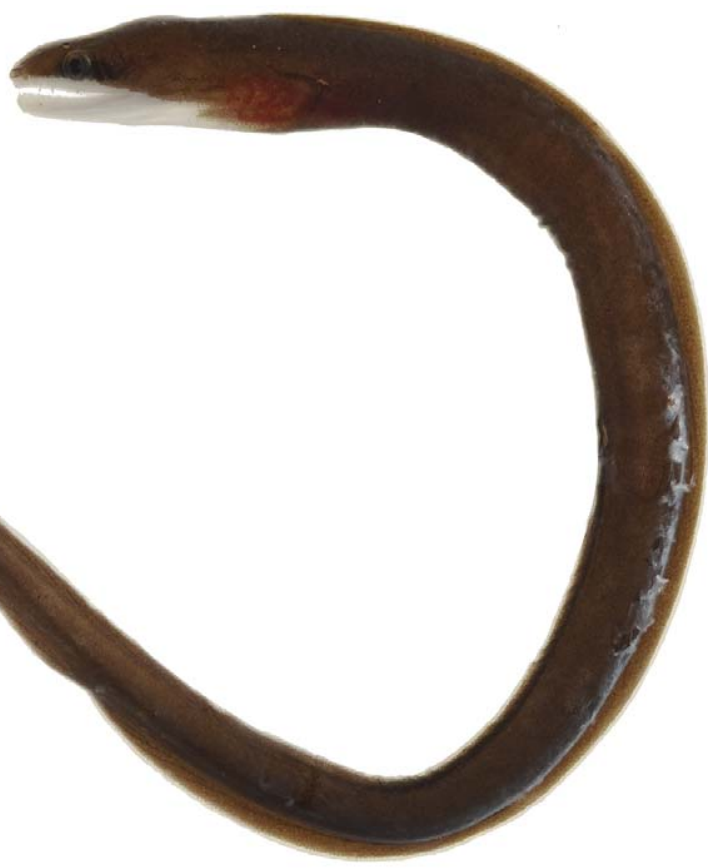

Figure 11. Gymnothorax vicinus, $48.2 \mathrm{~mm} \mathrm{TL}$, photo by JT Williams.

doi:10.1371/journal.pone.0010676.g011

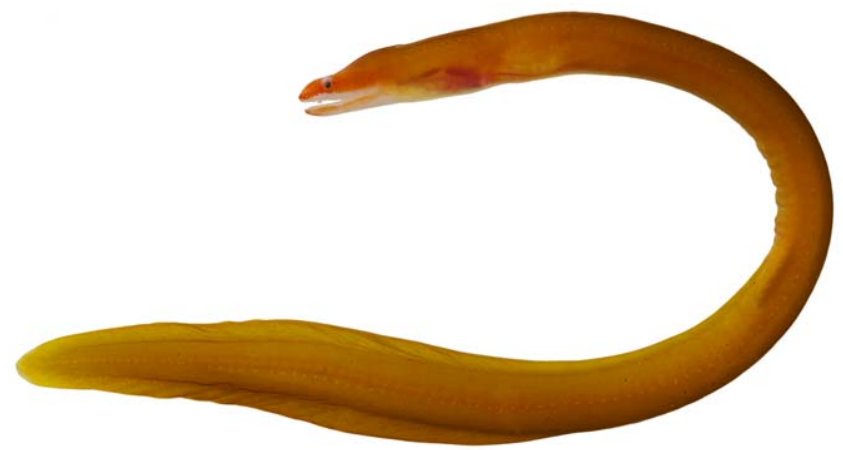

Figure 12. Monopenchelys acuta, $132.4 \mathrm{~mm} \mathrm{TL}$, photo by JT Williams.

doi:10.1371/journal.pone.0010676.g012 


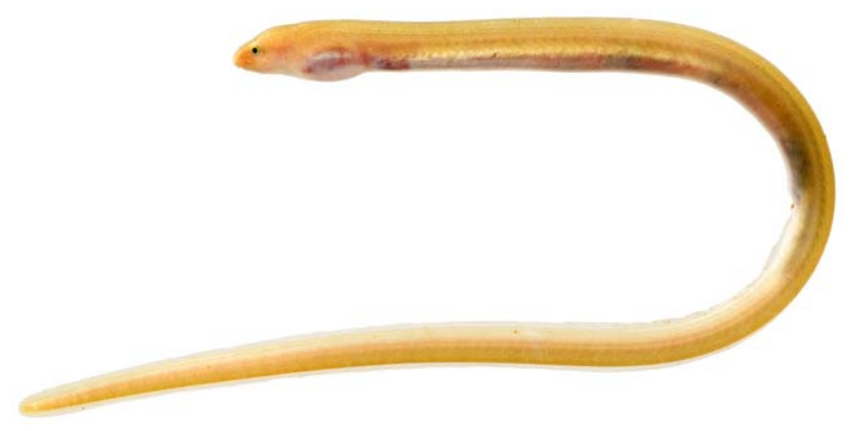

Figure 13. Ahlia egmontis, $183.0 \mathrm{~mm}$ TL, photo by JT Williams. doi:10.1371/journal.pone.0010676.g013

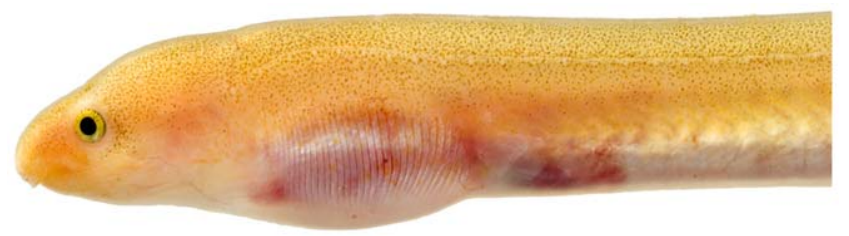

Figure 14. Ahlia egmontis, $183.0 \mathrm{~mm} \mathrm{TL}$, close-up of head, photo by JT Williams.

doi:10.1371/journal.pone.0010676.g014

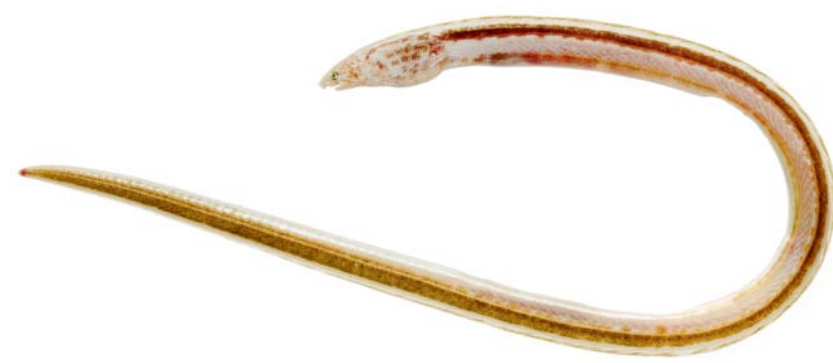

Figure 15. Aprognathodon platyventris, $149.2 \mathrm{~mm} \mathrm{TL}$, photo by JT Williams.

doi:10.1371/journal.pone.0010676.g015

Myrichthys ocellatus (Lesueur, 1825) — goldspotted eel; USNM, I; Figures 17, 18

\section{Congridae-conger eels}

Bathycongrus thysanochilus (Reid, 1934) - conger eel; USNM, T Conger esculentus Poey, 1866 - grey conger; USNM, F

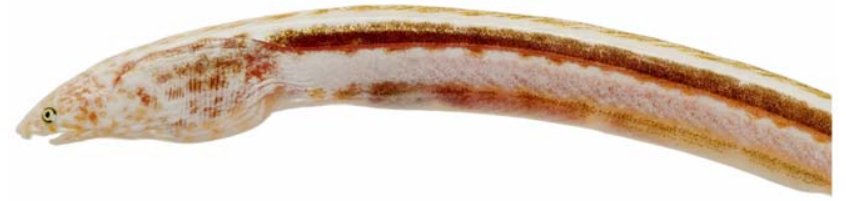

Figure 16. Aprognathodon platyventris, $149.2 \mathrm{~mm} \mathrm{TL}$, close-up of head, photo by JT Williams.

doi:10.1371/journal.pone.0010676.g016

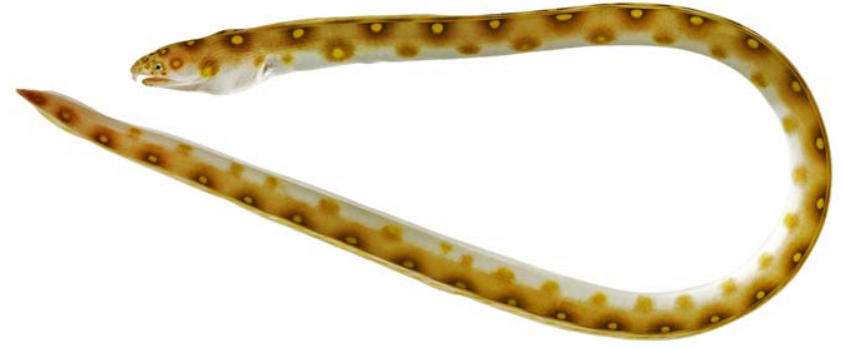

Figure 17. Myrichthys ocellatus, $383 \mathrm{~mm} \mathrm{TL}$, photo by JT Williams.

doi:10.1371/journal.pone.0010676.g017

Chlopsidae-false morays

Kaupichthys hyoproroides (Strömman, 1896) - false moray; USNM,

I; Figure 19

Kaupichthys nuchalis Böhlke, 1967-collared eel; USNM, I; Figure 20

Moringuidae-spaghetti eels

Moringua edwardsi (Jordan \& Bollman, 1889)—spaghetti eel; USNM, I; Figure 21

Synodontidae-lizardfishes

Saurida brasiliensis Norman 1935-largescale lizardfish; UF, T

Saurida normani Longley 1935 - shortjaw lizardfish; UF, T

Synodus intermedius (Spix \& Agassiz, 1829)— sand diver; USNM,

UF, F, O; Figure 22

Synodus poeyi Jordan, 1887 -offshore lizardfish; UF, T

Synodus saurus (Linnaeus, 1758)-Atlantic lizardfish; UF, I, V

Synodus synodus (Linnaeus, 1758) —red lizardfish; UF, USNM, I;

Figure 23

Trachinocephalus myops (Forster, 1801)—snakefish; V

Ophidiidae-cusk-eels

Brotula barbata (Bloch \& Schneider, 1801)-bearded brotula;

USNM, O; Figure 24

Neobythites ocellatus Günther 1887-ocellate cusk-eel; USNM, T

Neobythites unicolor Nielsen \& Retzer 1994-unicolor cusk-eel;

USNM, T

Ophidion antipholus Lea \& Robins, 2003-longnose cusk-eel;

UF, $\mathbf{T}$

Otophidium omostigma (Jordan \& Gilbert, 1882)—polka-dot cuskeel; UF, T

Parophidion schmidti (Woods \& Kanazawa, 1951)—dusky cusk-eel; USNM, I; Figure 25

Petrotyx sanguineus (Meek \& Hildebrand, 1928)—redfin brotula; USNM, I; Figure 26

Bythitidae-viviparous brotulas

Ogilbia sabaji Moller, Schwarzhans \& Nielsen, 2005-Sabaj coralbrotula; USNM, I; Figure 27

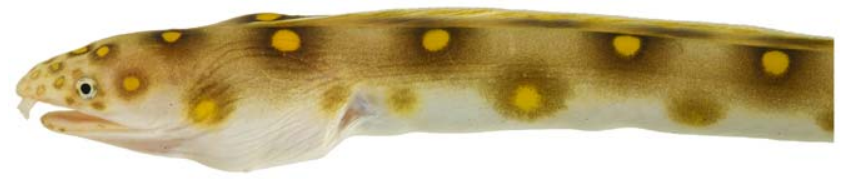

Figure 18. Myrichthys ocellatus, $383 \mathrm{~mm} \mathrm{TL}$, close-up of head, photo by JT Williams.

doi:10.1371/journal.pone.0010676.g018 


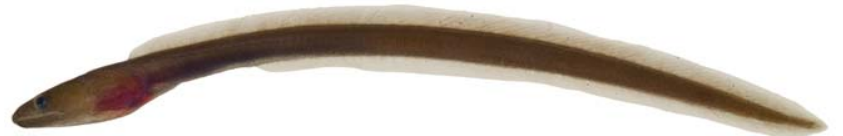

Figure 19. Kaupichthys hyoproroides, $79.4 \mathrm{~mm} \mathrm{TL}$, photo by JT Williams.

doi:10.1371/journal.pone.0010676.g019

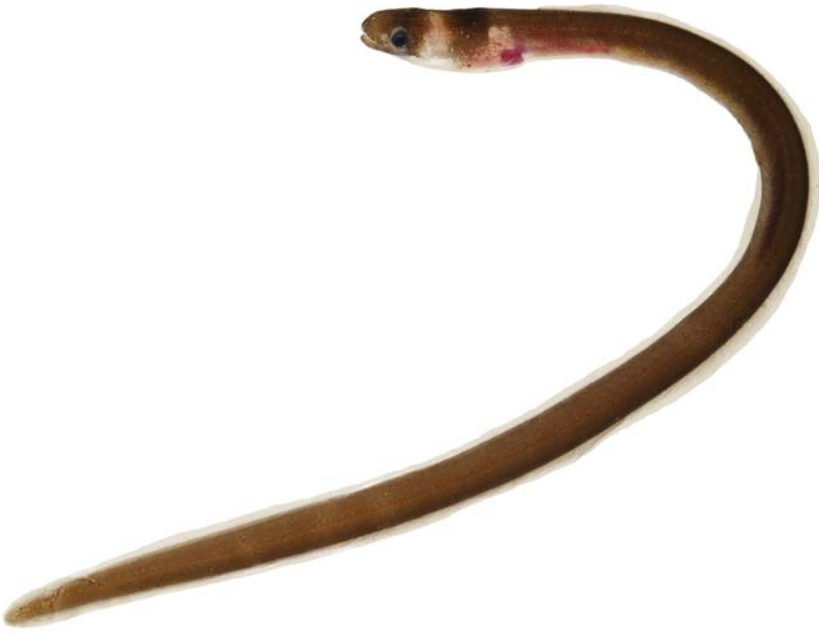

Figure 20. Kaupichthys nuchalisi, $61.6 \mathrm{~mm} \mathrm{TL}$, photo by JT Williams.

doi:10.1371/journal.pone.0010676.g020

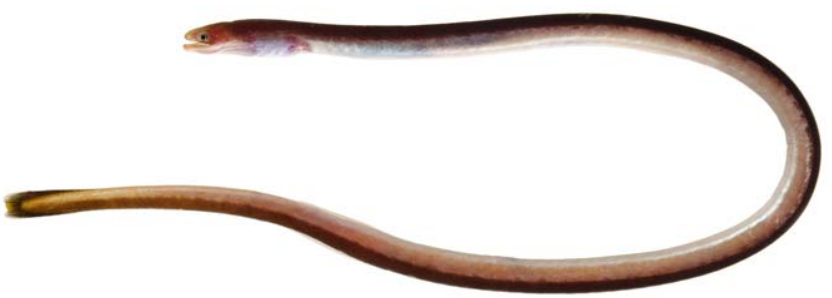

Figure 21. Moringua edwardsi, $258 \mathrm{~mm}$ TL, photo by JT Williams. doi:10.1371/journal.pone.0010676.g021

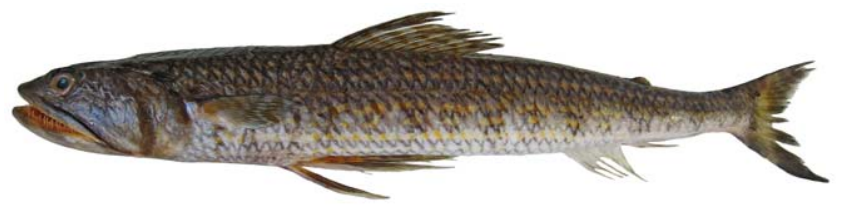

Figure 22. Synodus intermedius, $316 \mathrm{~mm} \mathrm{SL}$, photo by W Toller. doi:10.1371/journal.pone.0010676.g022

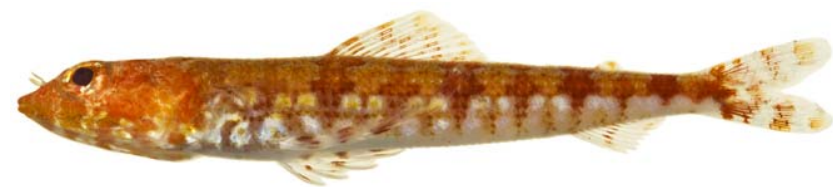

Figure 23. Synodus synodus, $42.5 \mathrm{~mm} \mathrm{SL}$, photo by JT Williams. doi:10.1371/journal.pone.0010676.g023

PLOS ONE I www.plosone.org

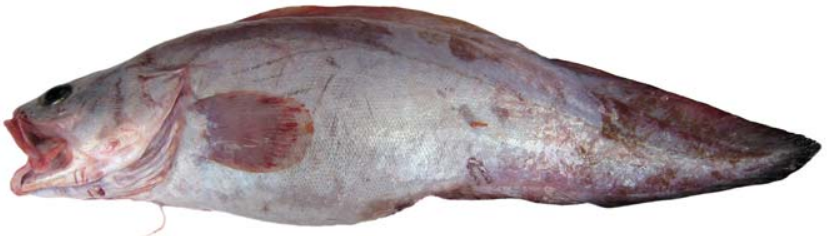

Figure 24. Brotula barbata, $628 \mathrm{~mm}$ TL, photo by W Toller. doi:10.1371/journal.pone.0010676.g024

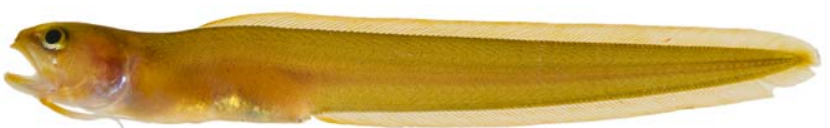

Figure 25. Parophidion schmidti, $69.3 \mathrm{~mm} \mathrm{TL}$, photo by JT Williams.

doi:10.1371/journal.pone.0010676.g025

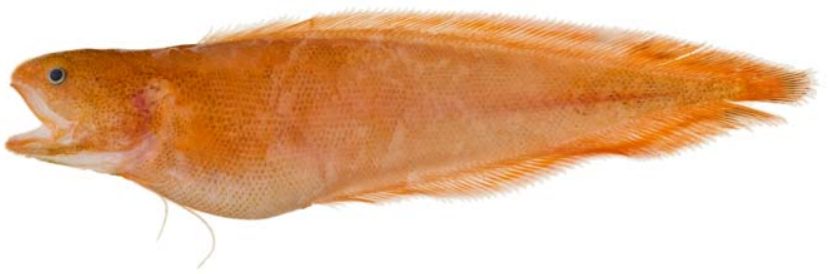

Figure 26. Petrotyx sanguineus, $62.2 \mathrm{~mm} \mathrm{TL}$, photo by JT Williams.

doi:10.1371/journal.pone.0010676.g026

Antennariidae-frogfishes

Antennarius pauciradiatus Schultz, 1957 —dwarf frogfish; USNM, I; Figures 28, 29

Antennarius multiocellatus (Valenciennes, 1837) - longlure frogfish; USNM, I

A single juvenile specimen was collected in 2007. Although adults of this species have a very long first spine, our juvenile specimen has the first dorsal spine about the same length as the second. Böhlke and Chaplin [3] mention this allometric growth pattern in which the first spine is short in young specimens, but increases in length with growth. The juvenile exhibits the typical adult color pattern.

Chaunacidae-sea toads

Chaunax suttkusi Caruso, 1989 - Suttkus sea toad; USNM, T

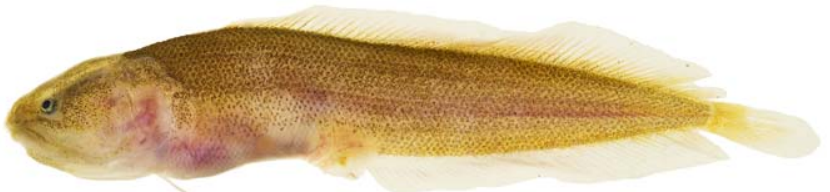

Figure 27. Ogilbia sabaji, $27.2 \mathrm{~mm} \mathrm{SL}$, photo by JT Williams. doi:10.1371/journal.pone.0010676.g027 


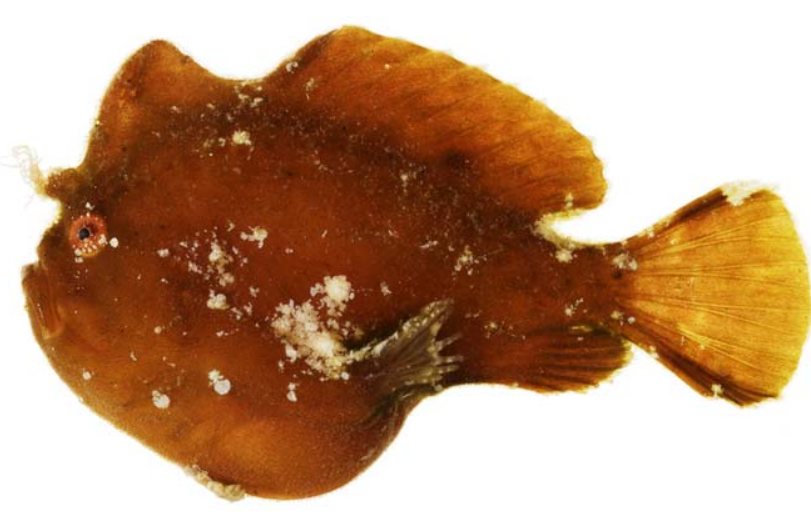

Figure 28. Antennarius pauciradiatus, $24.9 \mathrm{~mm} \mathrm{SL}$, photo by JT Williams.

doi:10.1371/journal.pone.0010676.g028

Ogcocephalidae-batfishes

All of the batfish records from Saba Bank are based on trawl collections with vouchered museum specimens.

Dibranchus atlanticus Peters, 1876-Atlantic batfish; UF, T

Halieutichthys aculeatus (Mitchill, 1818)_pancake batfish; UF, T Ogcocephalus pumilus Bradbury, 1980 — dwarf batfish; USNM, T

\section{Exocoetidae-flyingfishes}

Cypselurus comatus (Mitchill, 1815) — clearwing flyingfish; USNM

This specimen was probably captured at the surface using a dip net.

\section{Syngnathidae-pipefishes}

Anarchopterus tectus (Dawson, 1978) -insular pipefish; USNM, I Bryx randalli (Herald, 1965) —ocellated pipefish; USNM, I; Figure 30

Micrognathus crinitus (Jenyns, 1842) — banded pipefish; USNM, I;

Figures 31, 32

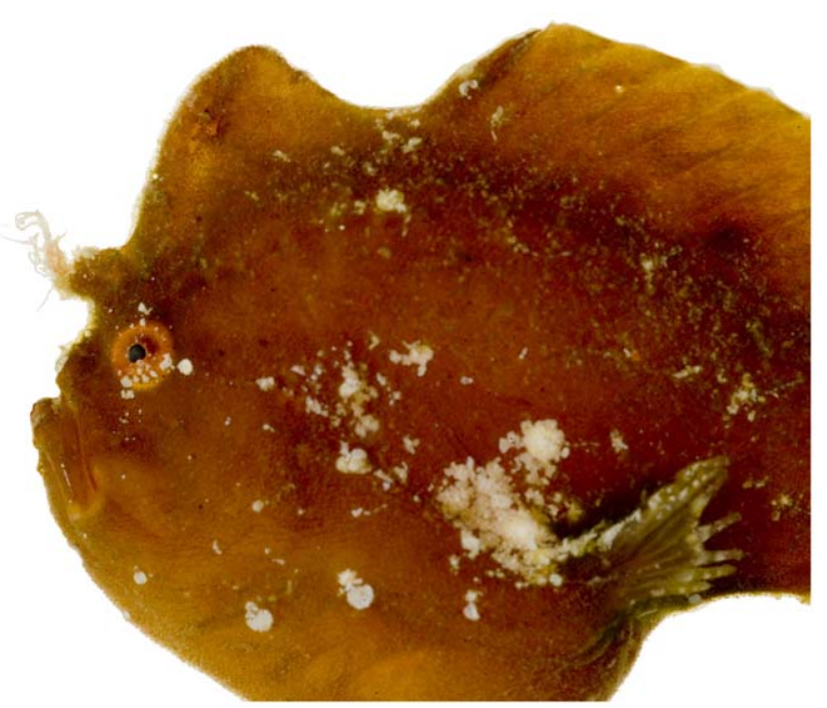

Figure 29. Antennarius pauciradiatus, $24.9 \mathrm{~mm} \mathrm{SL}$, close-up of head, photo by JT Williams.

doi:10.1371/journal.pone.0010676.g029

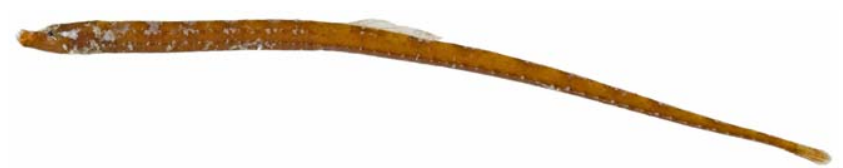

Figure 30. Bryx randalli, $24.3 \mathrm{~mm} \mathrm{SL}$, photo by JT Williams. doi:10.1371/journal.pone.0010676.g030

Figure 31. Micrognathus crinitus, $102.8 \mathrm{~mm} \mathrm{SL}$, photo by JT Williams.

doi:10.1371/journal.pone.0010676.g031

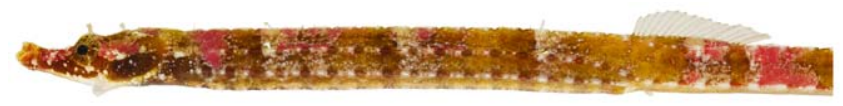

Figure 32. Micrognathus crinitus, $102.8 \mathrm{~mm} \mathrm{SL}$, close-up of head, photo by JT Williams.

doi:10.1371/journal.pone.0010676.g032

Aulostomidae-trumpetfishes

Aulostomus maculatus Valenciennes, 1837 -trumpetfish; USNM, $\mathbf{I}, \mathbf{O}, \mathbf{V}$

Holocentridae-squirrelfishes

Holocentrus adscensionis (Osbeck, 1765)-squirrelfish; USNM, I, $\mathbf{O}, \mathbf{V}$; Figure 33

Holocentrus rufus (Walbaum, 1792)-longspine squirrelfish;

USNM, I, O, V; Figure 34

Myripristis jacobus Cuvier, 1829 — blackbar soldierfish; USNM, I, o; Figure 35

Neoniphon marianus (Cuvier, 1829) - longjaw squirrelfish; USNM,

I; Figure 36

Plectrypops retrospinis (Guichenot, 1853) - cardinal soldierfish; USNM, I; Figure 37

Sargocentron coruscum (Poey, 1860) -reef squirrelfish; USNM, I, o; Figures 38, 39

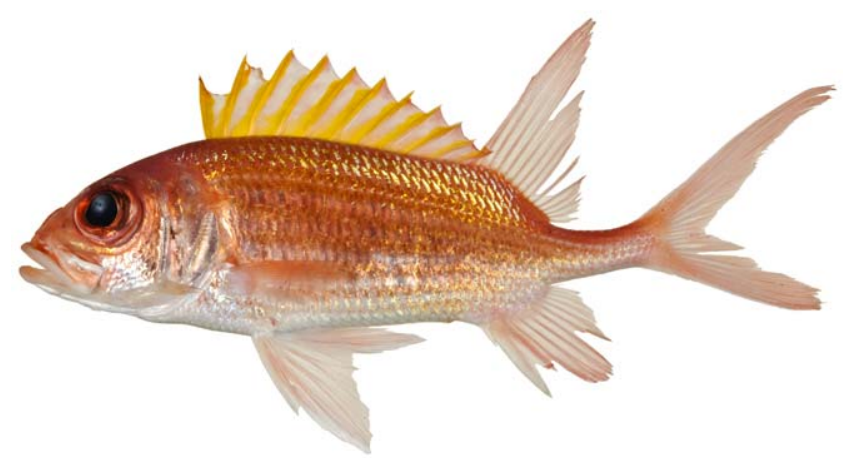

Figure 33. Holocentrus ascensionus, $195.0 \mathrm{~mm} \mathrm{SL}$, photo by JT Williams.

doi:10.1371/journal.pone.0010676.g033 


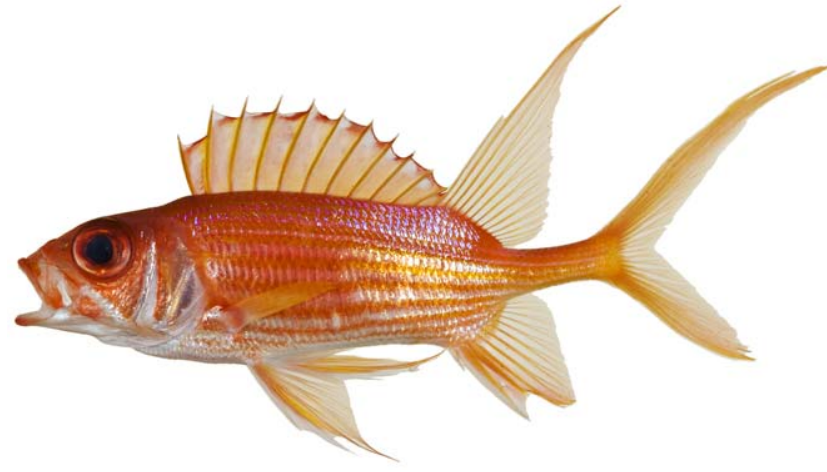

Figure 34. Holocentrus rufus, $153.2 \mathrm{~mm} \mathrm{SL}$, photo by JT Williams.

doi:10.1371/journal.pone.0010676.g034

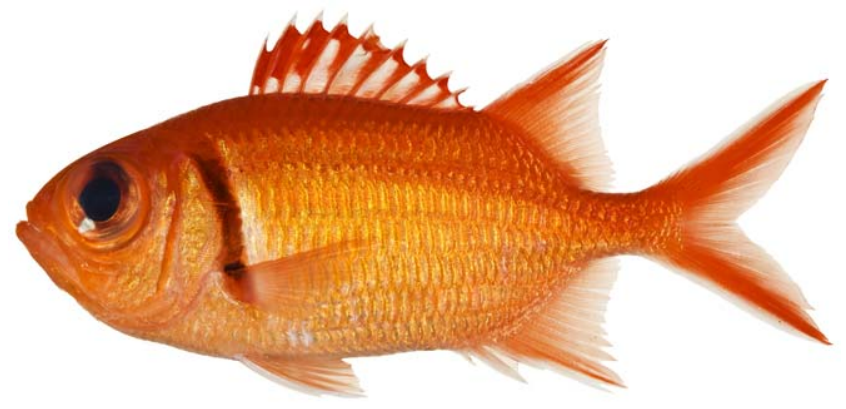

Figure 35. Myripristis jacobus, $114.7 \mathrm{~mm} \mathrm{SL}$, photo by JT Williams.

doi:10.1371/journal.pone.0010676.g035

\section{Scorpaenidae-scorpionfishes}

Scorpaena albifimbria Evermann \& Marsh, 1900 - coral scorpionfish; USNM, I; Figure 40

Scorpaena bergii Evermann \& Marsh, 1900-goosehead scorpionfish; USNM, I

Scorpaena grandicornis Cuvier, 1829-plumed scorpionfish; USNM, I; Figure 41

Scorpaena inermis Cuvier, 1829 mushroom scorpionfish; USNM, I, T; Figures 42, 43

Two color morphs were collected at Saba Bank, a red morph (Figure 42) and a yellow morph (Figure 43).

Scorpaena plumieri Bloch, 1789 - spotted scorpionfish; USNM, I

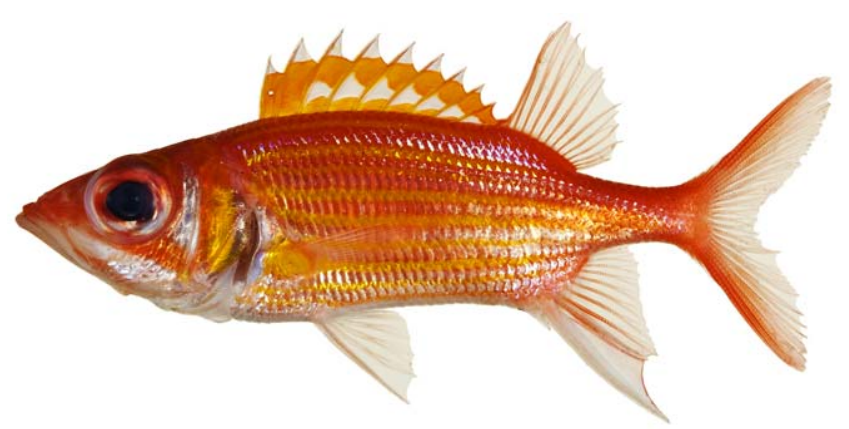

Figure 36. Neoniphon marianus, $98.2 \mathrm{~mm} \mathrm{SL}$, photo by JT Williams.

doi:10.1371/journal.pone.0010676.g036

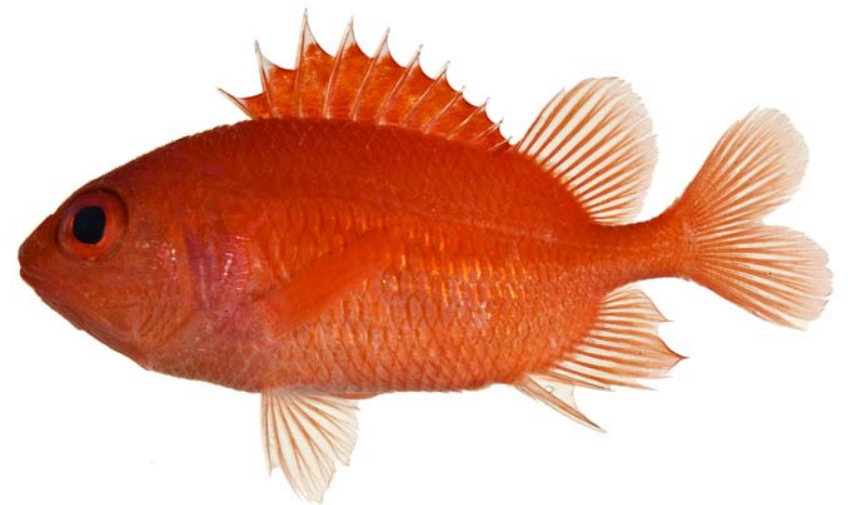

Figure 37. Plectrypops retrospinis, $71.5 \mathrm{~mm} \mathrm{SL}$, photo by JT Williams.

doi:10.1371/journal.pone.0010676.g037

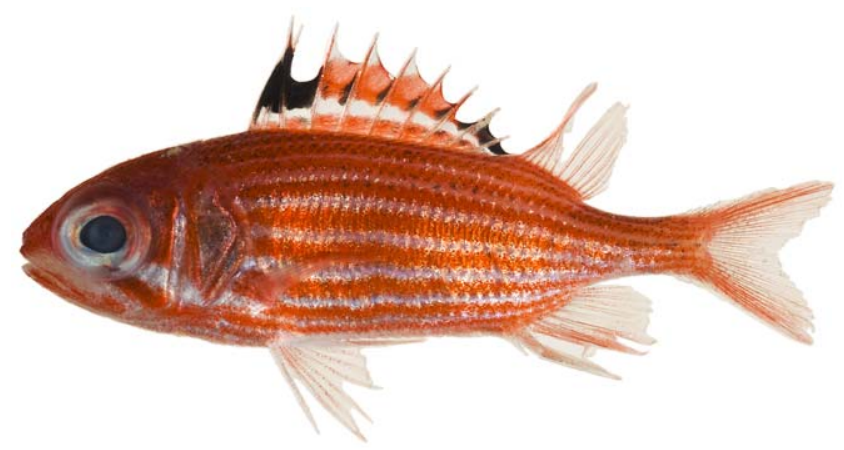

Figure 38. Sargocentron coruscum, juvenile, $31.1 \mathrm{~mm} \mathrm{SL}$, photo by JT Williams.

doi:10.1371/journal.pone.0010676.g038

Scorpaenodes caribbaeus Meek \& Hildebrand, 1928 - reef scorpionfish; USNM, I; Figure 44

Triglidae-searobins

All of the searobin records from Saba Bank are based on trawl collections with vouchered museum specimens.

Bellator egretta (Goode \& Bean, 1896)-streamer searobin; USNM, T

Bellator militaris (Goode \& Bean, 1896)—horned searobin; UF, T

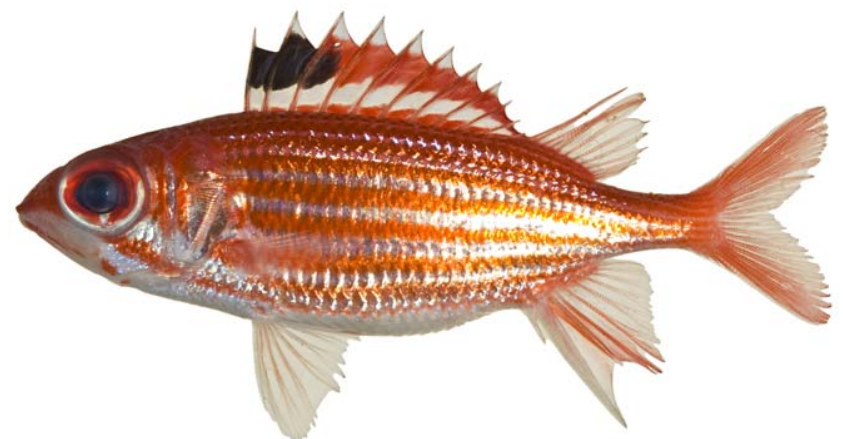

Figure 39. Sargocentron coruscum, $81.7 \mathrm{~mm} \mathrm{SL}$, photo by JT Williams.

doi:10.1371/journal.pone.0010676.g039 


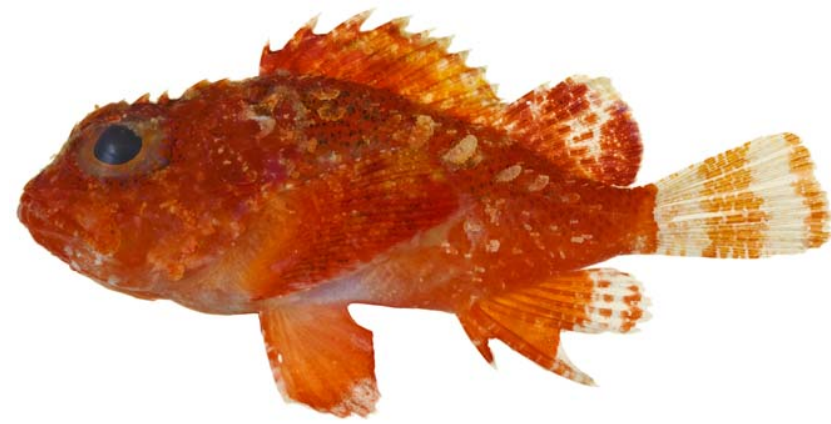

Figure 40. Scorpaena albifimbria, $37.4 \mathrm{~mm} \mathrm{SL}$, photo by JT Williams.

doi:10.1371/journal.pone.0010676.g040

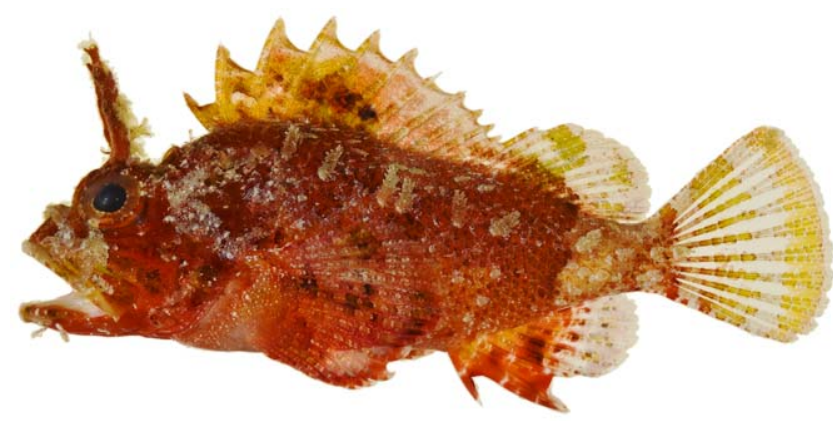

Figure 41. Scorpaena grandicornis, $65.1 \mathrm{~mm} \mathrm{SL}$, photo by JT Williams.

doi:10.1371/journal.pone.0010676.g041

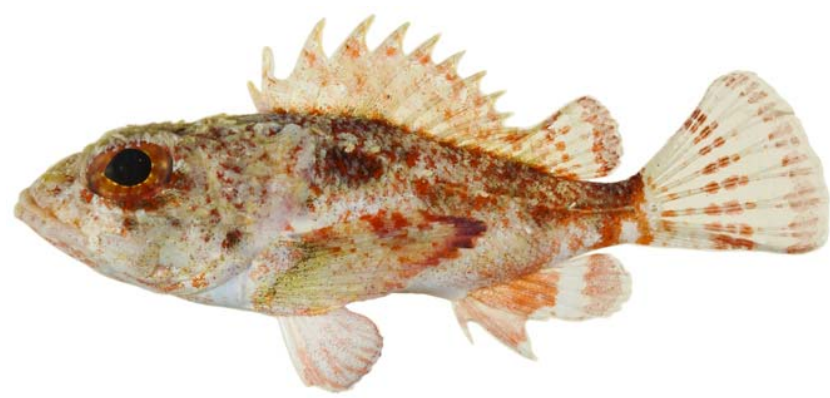

Figure 42. Scorpaena inermis, $40.1 \mathrm{~mm} \mathrm{SL}$, red morph, photo by JT Williams.

doi:10.1371/journal.pone.0010676.g042

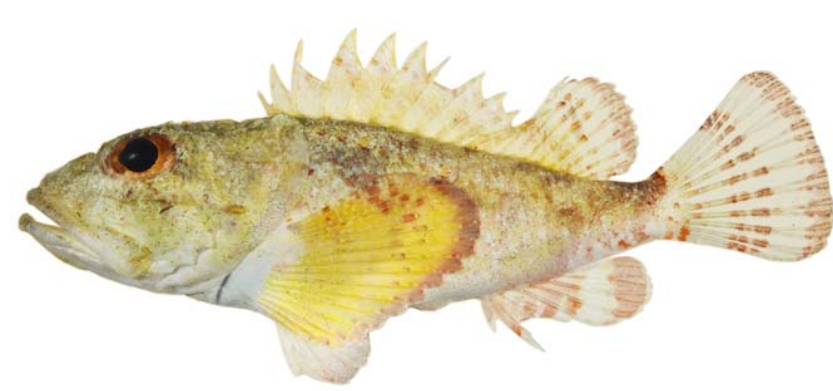

Figure 43. Scorpaena inermis, $65.9 \mathrm{~mm} \mathrm{SL}$, yellow morph, photo by JT Williams.

doi:10.1371/journal.pone.0010676.g043

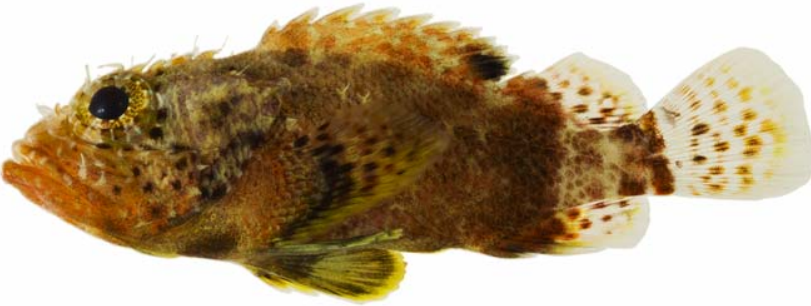

Figure 44. Scorpaenodes caribbaeus, $\mathbf{3 0 . 1} \mathbf{~ m m ~ S L , ~ p h o t o ~ b y ~ J T ~}$ Williams.

doi:10.1371/journal.pone.0010676.g044

Prionotus ophryas Jordan \& Swain, 1885-bandtail searobin; UF, T

Symphysanodontidae-slopefishes

Symphysanodon berryi Anderson, 1970 - slope bass; USNM, F; Figure 45

\section{Serranidae-sea basses}

Alphestes afer (Bloch, 1793) - mutton hamlet; F, O; Figure 46 Cephalopholis cruentata (Lacepède, 1802) - graysby; USNM, I, F,

$\mathbf{O}, \mathbf{V}$; Figure 47

Cephalopholis fulva (Linnaeus, 1758) - coney; UF, USNM, I, F, o, V; Figure 48

Diplectrum bivittatum (Valenciennes, 1828) — dwarf sand perch; UF, $\mathbf{T}$

Epinephelus flavolimbatus Poey, 1865-yellowedge grouper; F; Figure 49

Epinephelus guttatus (Linnaeus, 1758) —red hind; UF, USNM, F,

$\mathbf{I}, \mathbf{O}, \mathbf{T}, \mathbf{V}$; Figure 50

Epinephelus morio (Valenciennes, 1828) — red grouper; $\mathbf{F}$

Epinephelus niveatus Valenciennes, 1828- snowy grouper;

USNM, F; Figure 51

Epinephelus striatus (Bloch, 1792) -Nassau grouper; F

Hypoplectrus chlorurus (Cuvier, 1828) - yellowtail hamlet; $\mathbf{O}$

Hypoplectrus nigricans (Poey, 1852) - black hamlet; USNM, I, O,

V; Figure 52

Hypoplectrus puella (Cuvier, 1828) - barred hamlet; $\mathbf{O}, \mathbf{V}$

Liopropoma rubre Poey, 1861 - peppermint basslet; USNM, I; Figure 53

Mycteroperca interstitialis (Poey, 1860)-yellowmouth grouper; F; Figure 54

Mycteroperca tigris (Valenciennes, 1833)-tiger grouper; V

Mycteroperca venenosa (Linnaeus, 1758)-yellowfin grouper; F, OBS

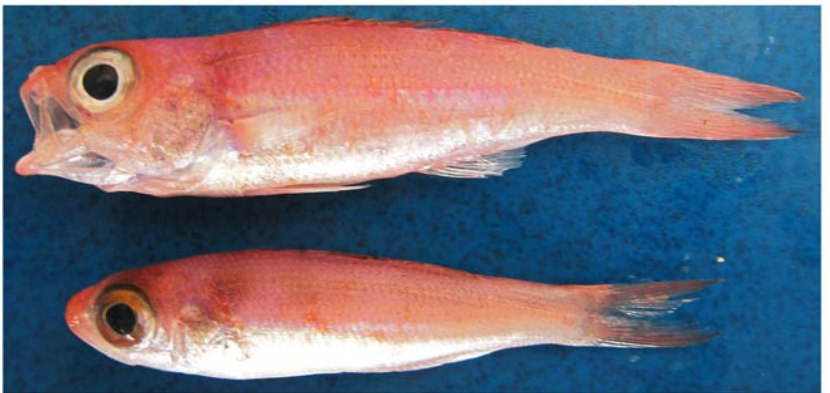

Figure 45. Symphysanodon berryi, top $75 \mathrm{~mm} \mathrm{SL}$, lower $63 \mathrm{~mm}$ SL, photo by W Toller.

doi:10.1371/journal.pone.0010676.g045 


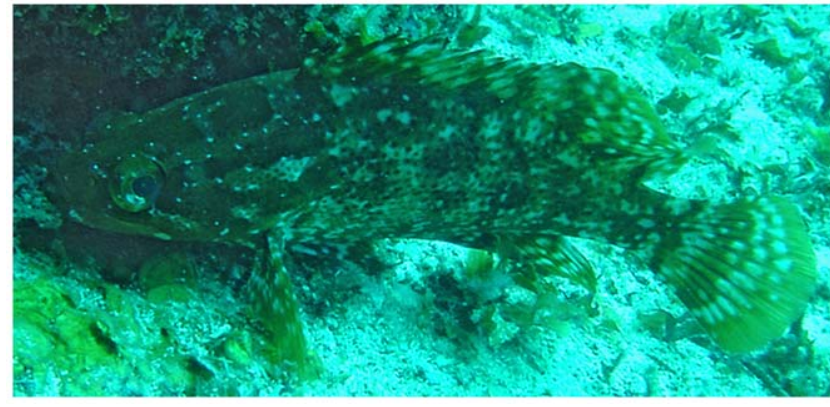

Figure 46. Alphestes afer, underwater photo by $\mathbf{W}$ Toller. doi:10.1371/journal.pone.0010676.g046

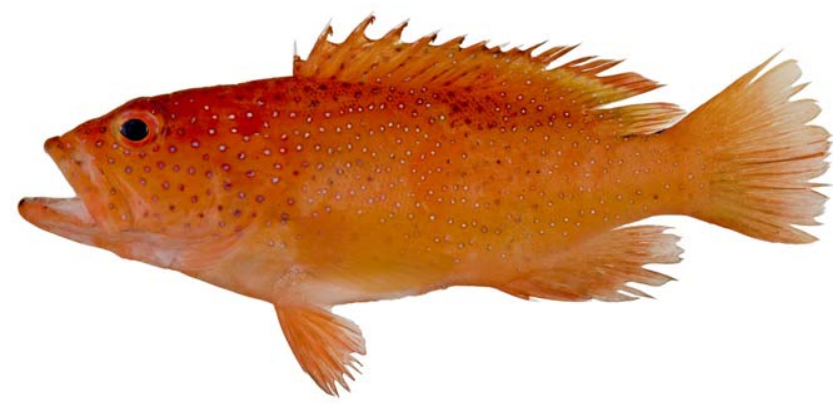

Figure 47. Cephalopholis cruentata, $101.1 \mathrm{~mm} \mathrm{SL}$, photo by JT Williams.

doi:10.1371/journal.pone.0010676.g047

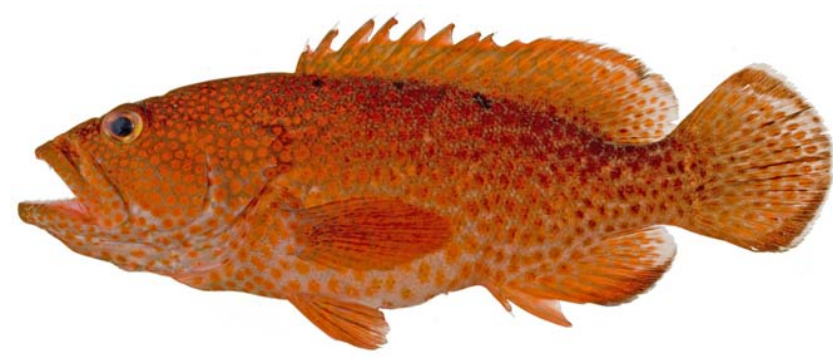

Figure 48. Cephalopholis fulva, $121.1 \mathrm{~mm}$ SL, photo by JT Williams.

doi:10.1371/journal.pone.0010676.g048

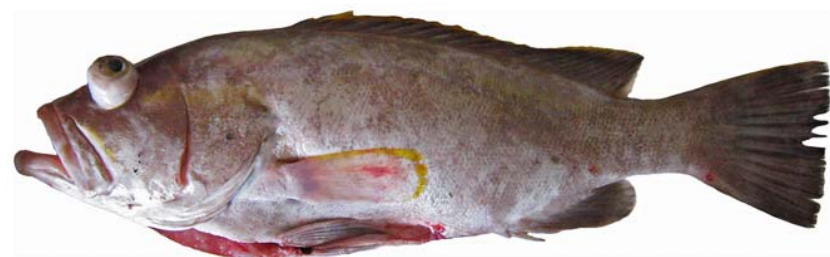

Figure 49. Epinephelus flavolimbatus, $652 \mathrm{~mm} \mathrm{SL}$, photo by $\mathrm{W}$ Toller.

doi:10.1371/journal.pone.0010676.g049

PLOS ONE I www.plosone.org

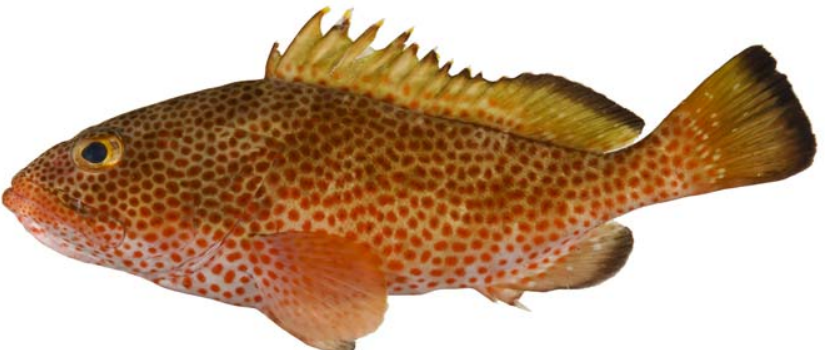

Figure 50. Epinephelus guttatus, $283.2 \mathrm{~mm} \mathrm{SL}$, photo by JT Williams.

doi:10.1371/journal.pone.0010676.g050

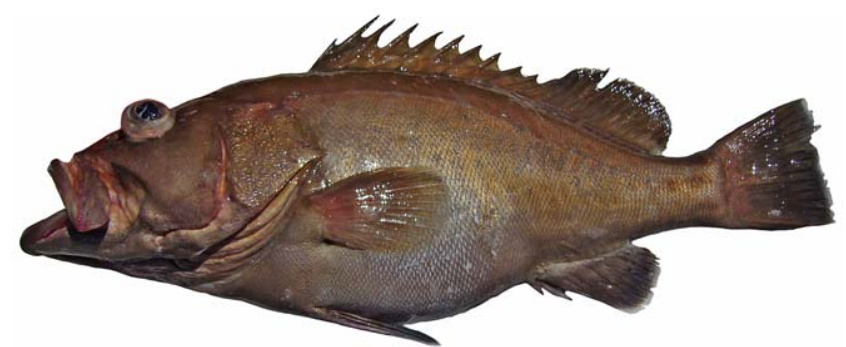

Figure 51. Epinephelus niveatus, $526 \mathrm{~mm} \mathrm{SL}$, photo by W Toller. doi:10.1371/journal.pone.0010676.g051

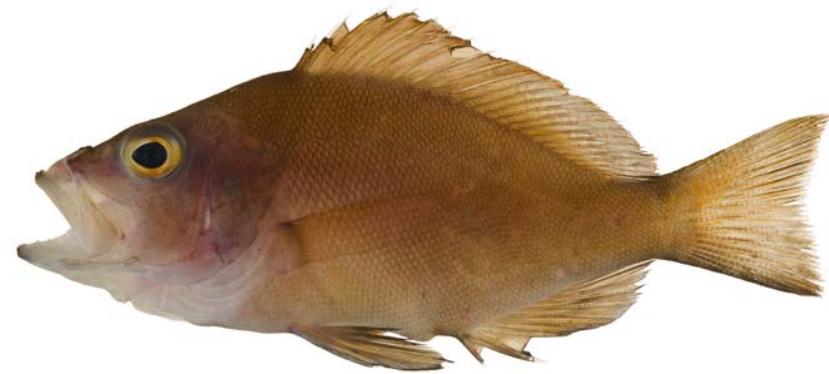

Figure 52. Hypoplectrus nigricans, $67.2 \mathrm{~mm} \mathrm{SL}$, photo by JT Williams.

doi:10.1371/journal.pone.0010676.g052

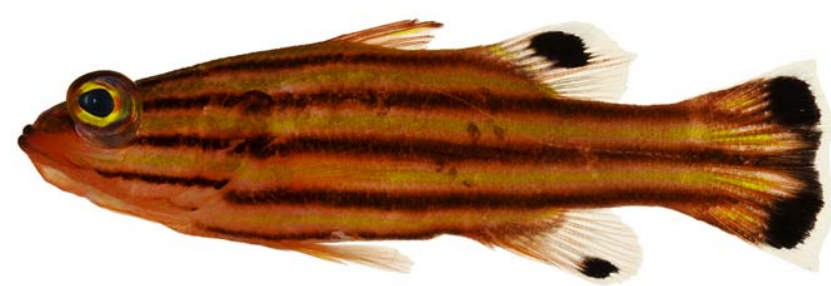

Figure 53. Liopropoma rubre, $\mathbf{3 0 . 0} \mathbf{~ m m ~ S L , ~ p h o t o ~ b y ~ J T ~ W i l l i a m s . ~}$ doi:10.1371/journal.pone.0010676.g053 


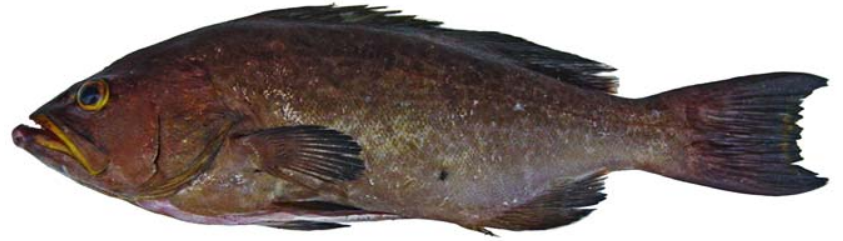

Figure 54. Mycteroperca interstitialis, $328 \mathrm{~mm} \mathrm{SL}$, photo by $\mathbf{W}$ Toller.

doi:10.1371/journal.pone.0010676.g054

Paranthias furcifer (Valenciennes, 1828)-Atlantic creolefish; UF, USNM, I, O, V; Figure 55

Pseudogramma gregoryi (Breder, 1927) - reef bass; USNM, I; Figure 56

Rypticus bistripinus (Mitchill, 1818) - freckled soapfish; USNM,

I; Figure 57

Rypticus saponaceus (Bloch \& Schneider, 1801) - greater soapfish;

USNM, I; Figure 58

Rypticus new species; USNM, I

This new species of soapfish is very similar in appearance to Rypticus subbifrenatus. The new species is being described by $\mathrm{C}$ Baldwin and DG Smith (pers. comm.).

Rypticus subbifrenatus Gill, 1861 - spotted soapfish; USNM, I;

Figure 59

Schultzea beta (Hildebrand, 1940) - school bass; UF, USNM, I

Serranus baldwini (Evermann \& Marsh, 1899)-lantern bass; UF,

USNM, I, O, V; Figure 60

Serranus maytagi Robins \& Starck, 1961 -maytag bass; UF

Serranus notospilus Longley, 1935 - saddle bass; USNM, F;

Figure 61

Serranus tabacarius (Cuvier, 1829) - tobaccofish; UF, O, V

Serranus tigrinus (Bloch, 1790) - harlequin bass; USNM, I, O, V;

Figure 62

Serranus tortugarum Longley, 1935 - chalk bass; UF, USNM, I, o; Figure 63

\section{Grammatidae-basslets}

Gramma loreto Poey, 1868 - fairy basslet; USNM, I, O, V; Figure 64

\section{Opistognathidae-jawfishes}

Opistognathus aurifrons (Jordan \& Thompson, 1905)—yellowhead jawfish; USNM, I, O, V; Figure 65

Opistognathus whitehursti (Longley, 1927) — dusky jawfish; USNM,

I; Figures 66, 67

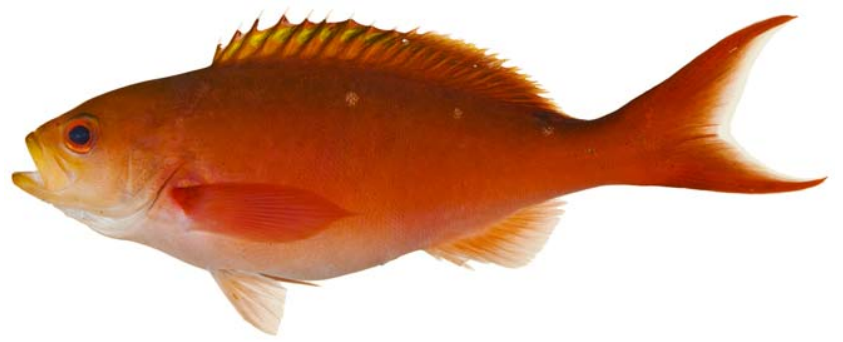

Figure 55. Paranthias furcifer, $148.4 \mathrm{~mm} \mathrm{SL}$, photo by JT Williams.

doi:10.1371/journal.pone.0010676.g055

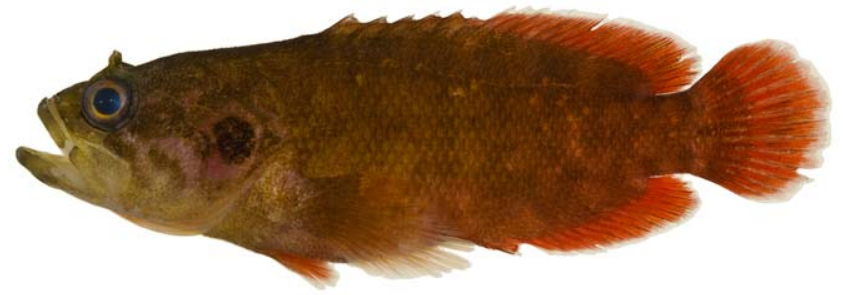

Figure 56. Pseudogramma gregoryi, $43.5 \mathrm{~mm} \mathrm{SL}$, photo by JT Williams.

doi:10.1371/journal.pone.0010676.g056

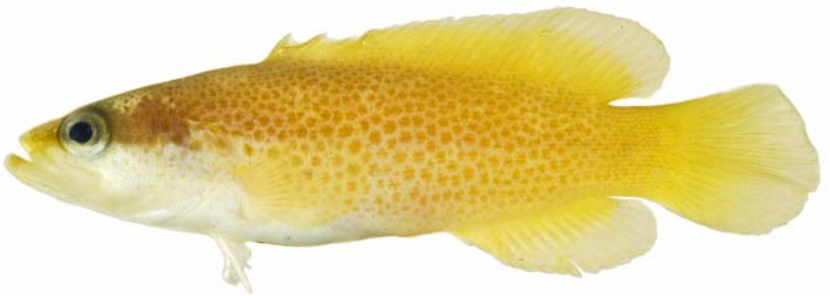

Figure 57 Rypticus bistripinus, $46.2 \mathrm{~mm} \mathrm{SL}$, photo by JT Williams.

doi:10.1371/journal.pone.0010676.g057

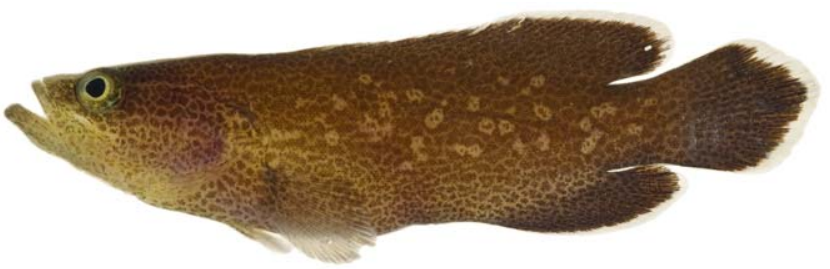

Figure 58. Rypticus saponaceus, $44.1 \mathrm{~mm} \mathrm{SL}$, photo by JT Williams.

doi:10.1371/journal.pone.0010676.g058

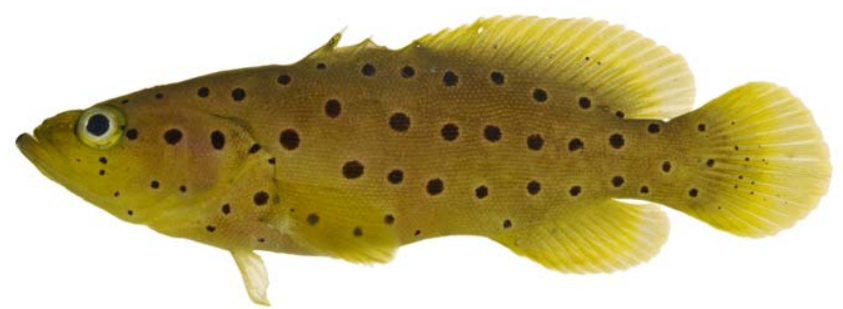

Figure 59. Rypticus subbifrenatus, $54.0 \mathrm{~mm} \mathrm{SL}$, photo by JT Williams.

doi:10.1371/journal.pone.0010676.g059 


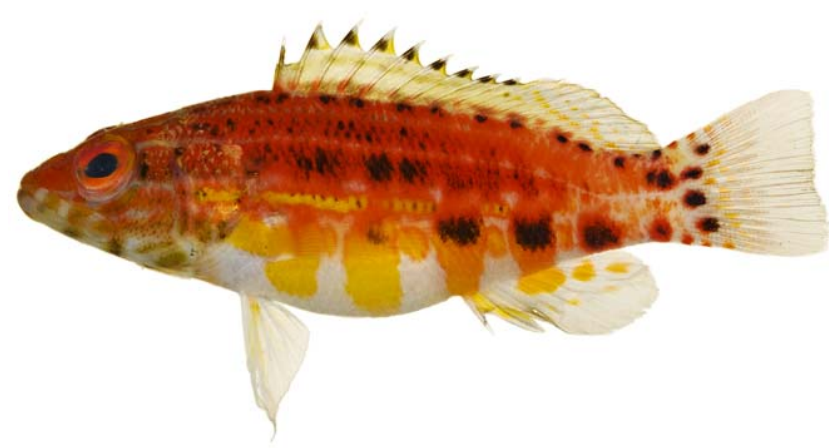

Figure 60. Serranus baldwini, $41.6 \mathrm{~mm} \mathrm{SL}$, photo by JT Williams. doi:10.1371/journal.pone.0010676.g060

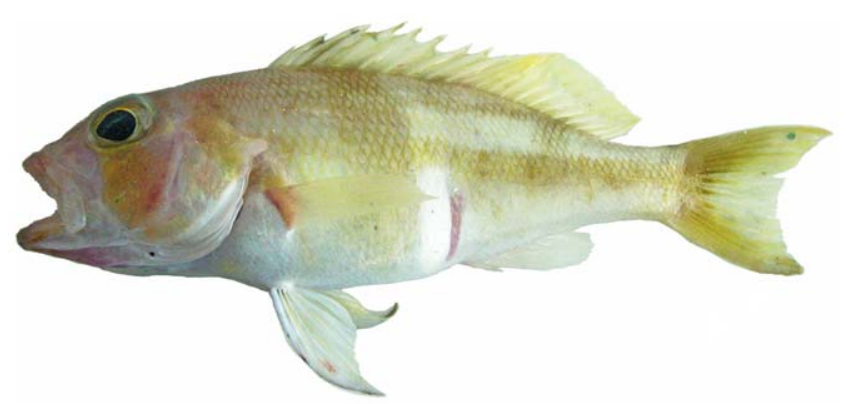

Figure 61. Serranus notospilus, $150 \mathrm{~mm} \mathrm{SL}$, photo by W Toller. doi:10.1371/journal.pone.0010676.g061

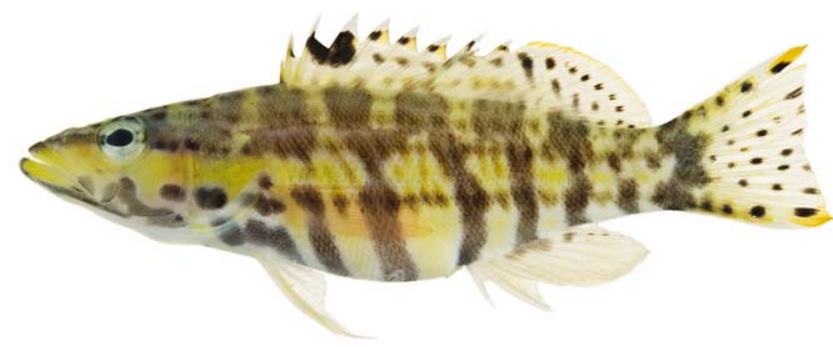

Figure 62. Serranus tigrinus, $71.1 \mathrm{~mm} \mathrm{SL}$, photo by JT Williams. doi:10.1371/journal.pone.0010676.g062

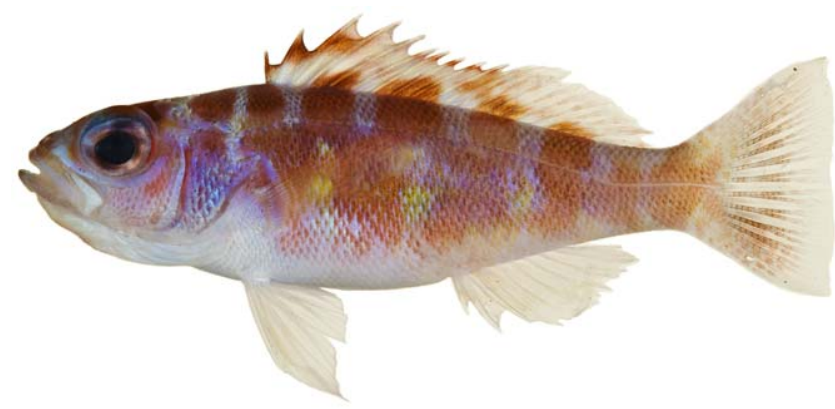

Figure 63. Serranus tortugarum, $51.8 \mathrm{~mm} \mathrm{SL}$, photo by JT Williams.

doi:10.1371/journal.pone.0010676.g063

PLOS ONE I www.plosone.org
Figure 64. Gramma loreto, $38.4 \mathrm{~mm} \mathrm{SL}$, photo by JT Williams. doi:10.1371/journal.pone.0010676.g064

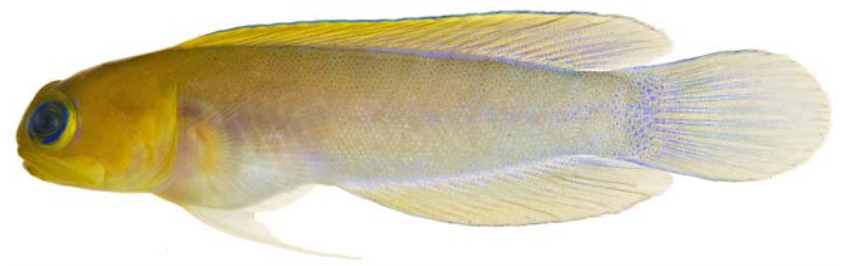

Figure 65. Opistognathus aurifrons, $44.1 \mathrm{~mm} \mathrm{SL}$, photo by JT Williams.

doi:10.1371/journal.pone.0010676.g065

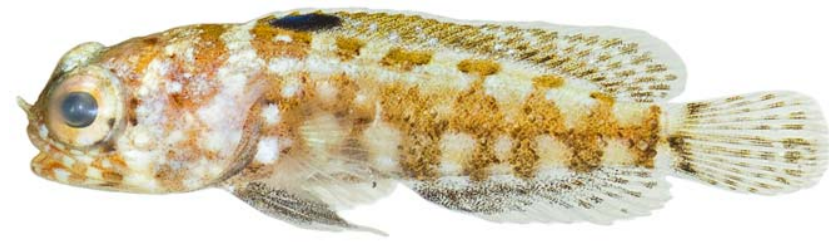

Figure 66. Opistognathus whitehursti, juvenile with large black spot in dorsal fin, $22.1 \mathrm{~mm} \mathrm{SL}$, photo by JT Williams. doi:10.1371/journal.pone.0010676.g066

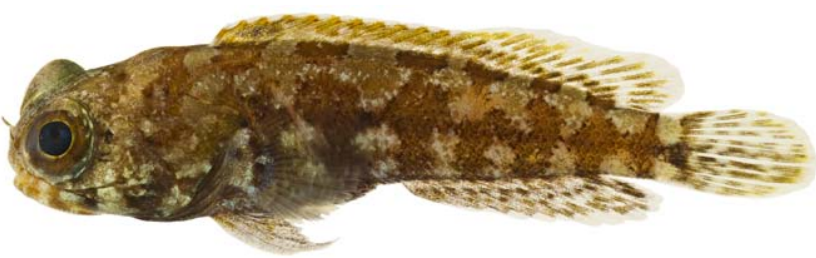

Figure 67. Opistognathus whitehursti, adult, $44.0 \mathrm{~mm} \mathrm{SL}$, photo by JT Williams.

doi:10.1371/journal.pone.0010676.g067 
Priacanthidae-bigeyes

$\mathbf{O}$

Heteropriacanthus cruentatus (Lacepède, 1801) — glasseye snapper;

Priacanthus arenatus Cuvier, 1829 - bigeye; USNM, I; Figure 68

Apogonidae-cardinalfishes

Apogon aurolineatus (Mowbray in Breder, 1927) — barred cardinal-

fish; USNM, I

Apogon binotatus (Poey, 1867) - barred cardinalfish; USNM, I

Apogon maculatus (Poey, 1860) - flamefish; USNM, I; Figure 69

Apogon pillionatus Böhlke and Randall, 1968 broadsaddle cardinalfish; UF, USNM, I

Apogon cf quadrisquamatus Longley, 1934 _sawcheek cardinalfish; USNM, I; Figure 70

The specimens identified here as A.cf quadrisquamatus have been found to represent a new undescribed species closely related to $A$. quadrisquamatus (C. Baldwin \& D.G. Smith, pers. comm. 2009).

Apogon robinsi Böhlke \& Randall, 1968 roughlip cardinalfish;

USNM, I; Figure 71

Apogon townsendi (Breder, 1927) — belted cardinalfish; USNM, I;

Figure 72

Astrapogon puncticulatus (Poey, 1867) - blackfin cardinalfish;

USNM, I; Figure 73

Phaeoptyx conklini (Silvester, 1916)_freckled cardinalfish;

USNM, I; Figure 74

Phaeoptyx pigmentaria (Poey, 1860) - dusky cardinalfish; USNM, I

Malacanthidae-tilefishes

Caulolatilus cyanops Poey, 1866-blackline tilefish; USNM, F; Figure 75

Malacanthus plumieri (Bloch, 1786) — sand tilefish; UF, O, V

\section{Coryphaenidae-dolphinfishes}

Coryphaena hippurus Linnaeus, 1758-common dolphinfish; $\mathbf{F}$

\section{Rachycentridae-cobias}

Rachycentron canadum (Linnaeus, 1766) - cobia

Our record for this species is based on an underwater video recently filmed by Yap Films Inc (Toronto, Canada) at the shipwreck on Saba Bank. The video clearly shows a cobia swimming along the side of the shipwreck.

\section{Carangidae-jacks}

Alectis ciliaris (Bloch, 1787)-African pompano; O; Figure 76 Caranx bartholomaei Cuvier, 1833-yellow jack; O, V

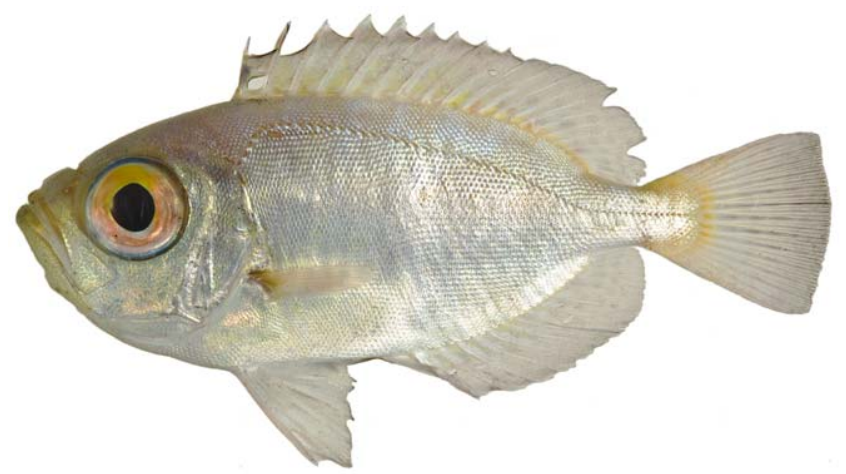

Figure 68. Priacanthus arenatus, $67.4 \mathrm{~mm} \mathrm{SL}$, photo by JT Williams.

doi:10.1371/journal.pone.0010676.g068

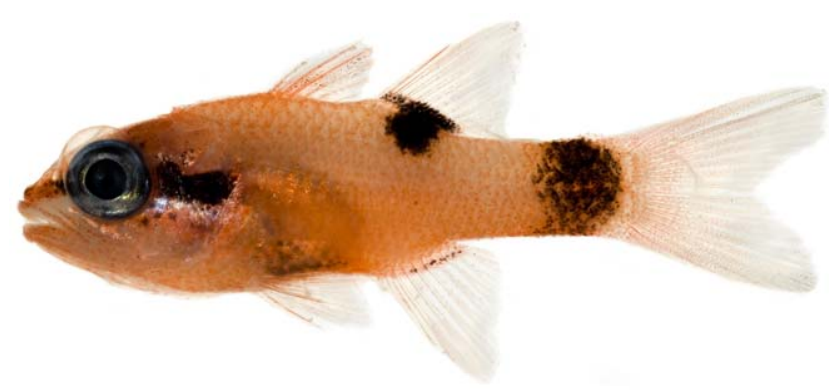

Figure 69. Apogon maculatus, $21.1 \mathrm{~mm} \mathrm{SL}$, photo by JT Williams. doi:10.1371/journal.pone.0010676.g069

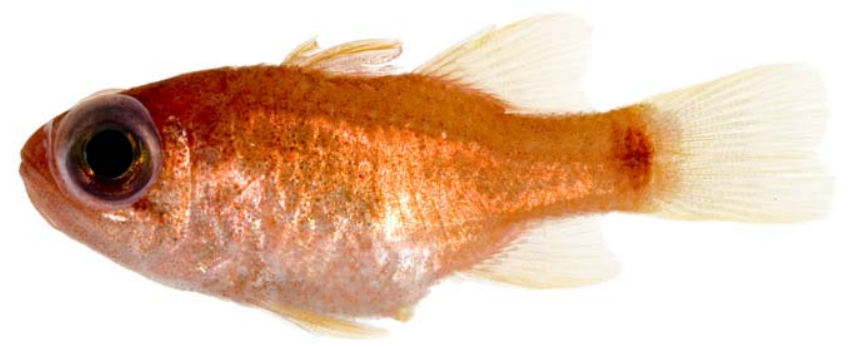

Figure 70. Apogon of quadrisquamatus, $21.1 \mathrm{~mm} \mathrm{SL}$, photo by JT Williams (this specimen represents a new undescribed species. doi:10.1371/journal.pone.0010676.g070

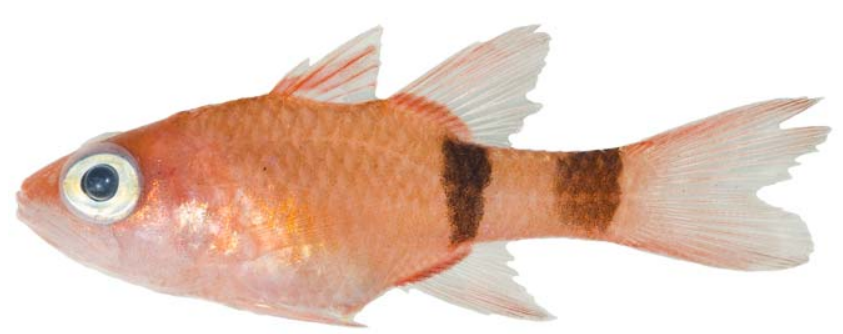

Figure 71. Apogon robinsi, $35.5 \mathrm{~mm} \mathrm{SL}$, photo by JT Williams. doi:10.1371/journal.pone.0010676.g071

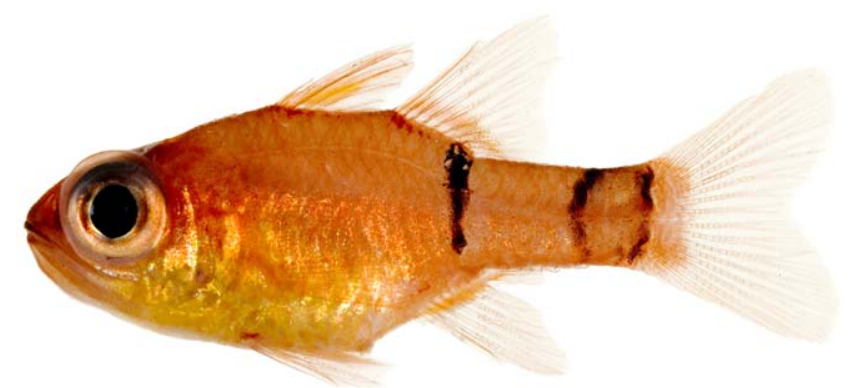

Figure 72. Apogon townsendi, $39.1 \mathrm{~mm} \mathrm{SL}$, photo by JT Williams.

doi:10.1371/journal.pone.0010676.g072 


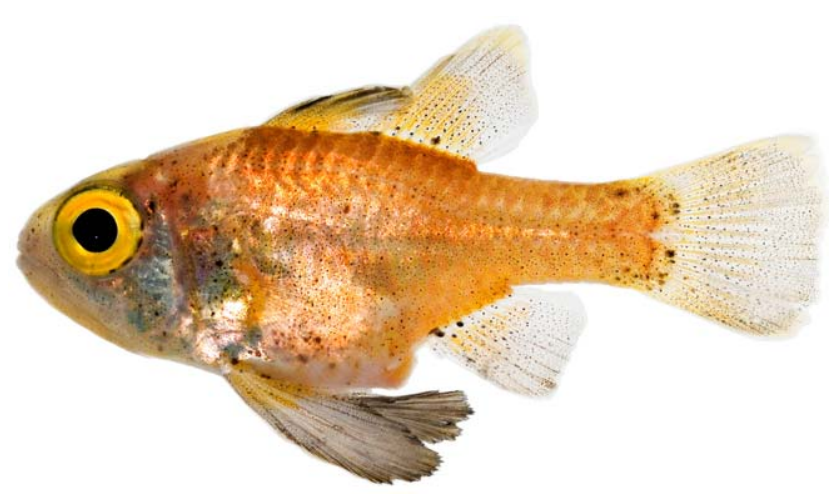

Figure 73. Astrapogon puncticulatus, $40.0 \mathrm{~mm} \mathrm{SL}$, photo by JT Williams.

doi:10.1371/journal.pone.0010676.g073

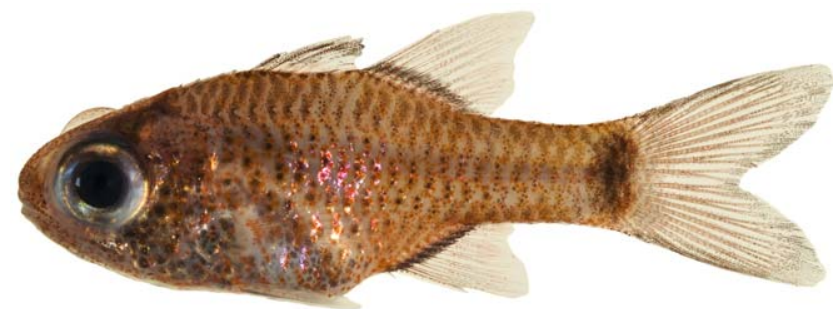

Figure 74. Phaeoptyx conklini, $40.0 \mathrm{~mm} \mathrm{SL}$, photo by JT Williams.

doi:10.1371/journal.pone.0010676.g074

Caranx crysos (Mitchill, 1815) -blue runner; $\mathbf{O}$

Caranx latus Agassiz, 1831 - horse-eye jack; O, V

Caranx lugubris Poey, 1860 - black jack; $\mathbf{O}, \mathbf{V}$

Caranx ruber (Bloch, 1793) - bar jack; UF, F, O, V; Figure 77

Decapterus macarellus (Cuvier, 1833) -mackerel scad; O; Figure 78

Elagatis bipinnulata (Quoy \& Gaimard, 1825) - rainbow runner;

\section{F, OBS}

Selar crumenophthalmus (Bloch, 1793) — bigeye scad; F; Figure 79 Seriola dumerili (Risso, 1810) - greater amberjack; F; Figure 80

Seriola rivoliana Valenciennes, 1833 - almaco jack; F; Figure 80

\section{Lutjanidae-snappers}

Apsilus dentatus Guichenot, 1853-black snapper; USNM, F;

Figure 81

Etelis oculatus (Valenciennes, 1828) -queen snapper; F

Lutjanus apodus (Walbaum, 1792) -schoolmaster; O, VIS

Lutjanus buccanella (Cuvier, 1828)_blackfin snapper; USNM, F,

O; Figure 82

Lutjanus cyanopterus (Cuvier, 1828)—cubera snapper; USNM, I

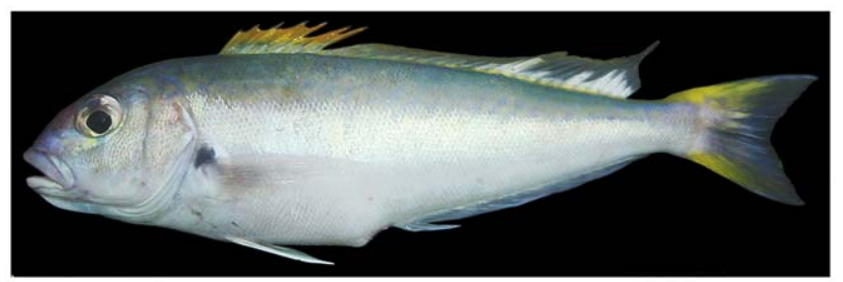

Figure 75. Caulolatilus cyanops, $288 \mathrm{~mm} \mathrm{SL}$, photo by W Toller. doi:10.1371/journal.pone.0010676.g075

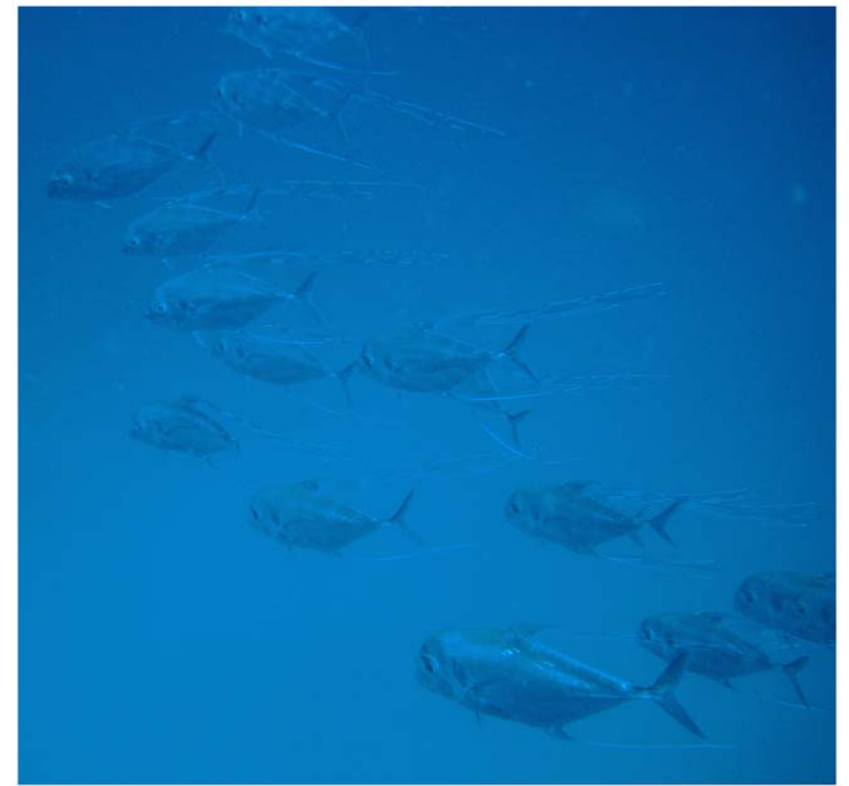

Figure 76. Alectis ciliaris, underwater photo by W Toller. doi:10.1371/journal.pone.0010676.g076

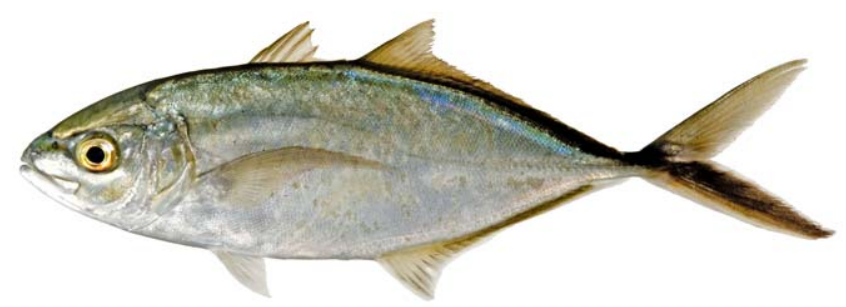

Figure 77. Caranx ruber, $\mathbf{2 6 0 . 8} \mathbf{~ m m ~ S L , ~ p h o t o ~ b y ~ J T ~ W i l l i a m s . ~}$ doi:10.1371/journal.pone.0010676.g077

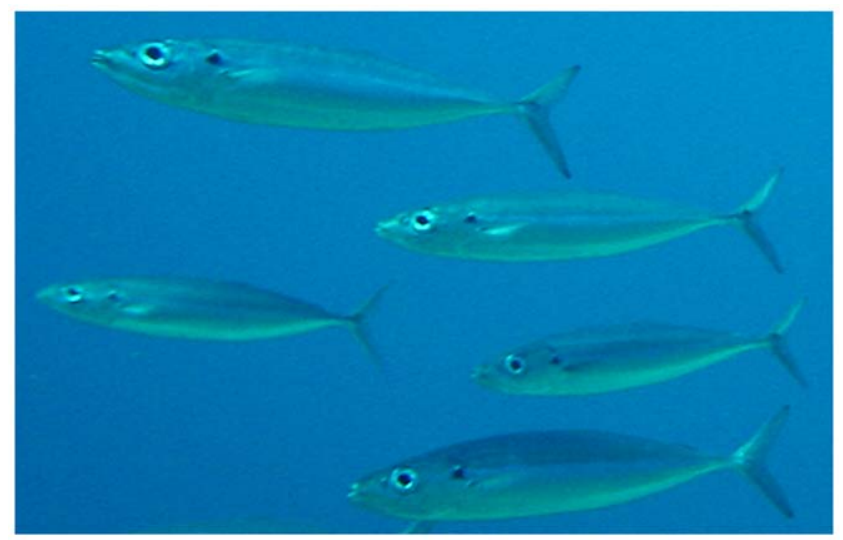

Figure 78. Decapterus macarellus, underwater photo by W Toller. doi:10.1371/journal.pone.0010676.g078 


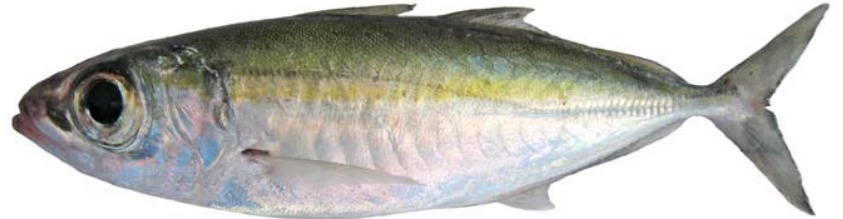

Figure 79. Selar crumenophthalmus, $240 \mathrm{~mm} \mathrm{SL}$, photo by W Toller.

doi:10.1371/journal.pone.0010676.g079

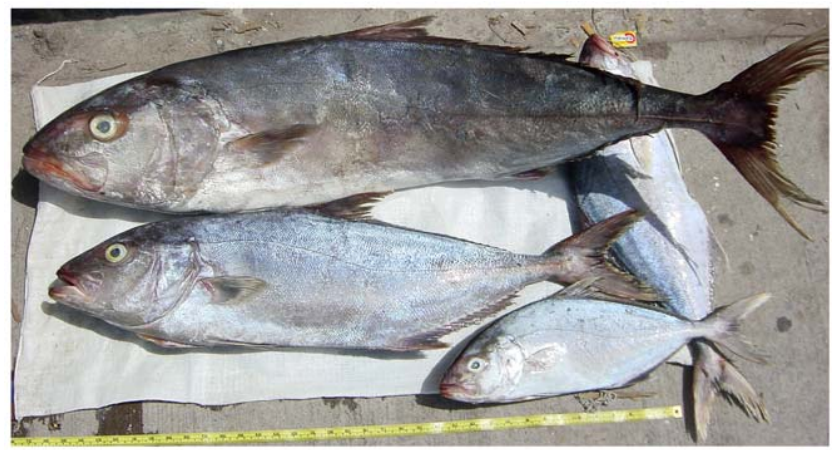

Figure 80. Seriola dumerili, large specimen at top; Seriola rivoliana, two smaller specimens below, photo by $\mathbf{W}$ Toller. doi:10.1371/journal.pone.0010676.g080

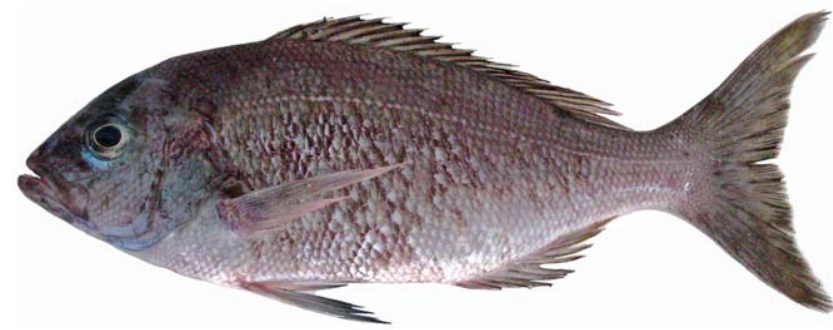

Figure 81. Apsilus dentatus, 234 mm SL, photo by W Toller. doi:10.1371/journal.pone.0010676.g081

Lutjanus mahogoni (Cuvier, 1828) —mahogany snapper; $\mathbf{O}$

Lutjanus purpureus (Poey, 1866)_Caribbean red snapper; USNM, F; Figure 83

Lutjanus synagris (Linnaeus, 1758)-lane snapper; USNM, F; Figure 84

Lutjanus vivanus (Guvier, 1828) -silk snapper; USNM, F; Figure 85

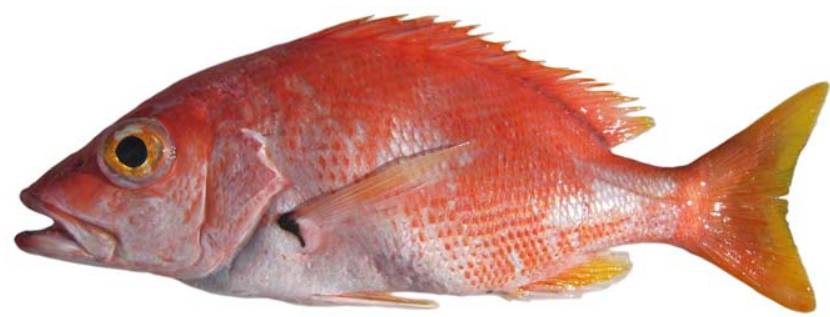

Figure 82. Lutjanus buccanella, 249 mm SL, photo by W Toller. doi:10.1371/journal.pone.0010676.g082

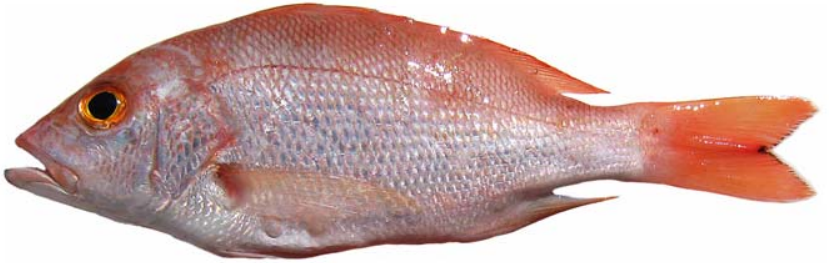

Figure 83. Lutjanus purpureus, 207 mm SL, photo by W Toller. doi:10.1371/journal.pone.0010676.g083

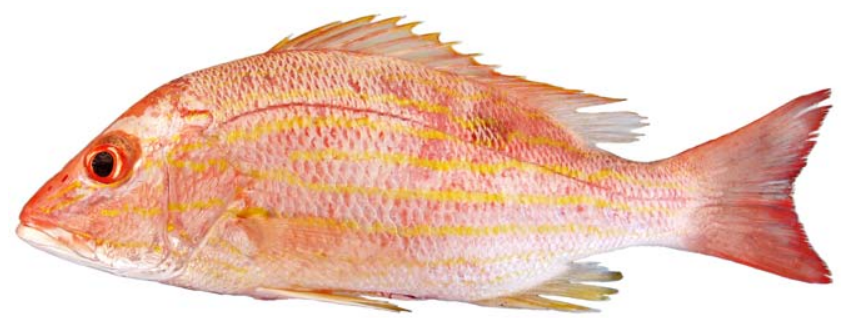

Figure 84. Lutjanus synagris, 208 mm SL, photo by W Toller. doi:10.1371/journal.pone.0010676.g084

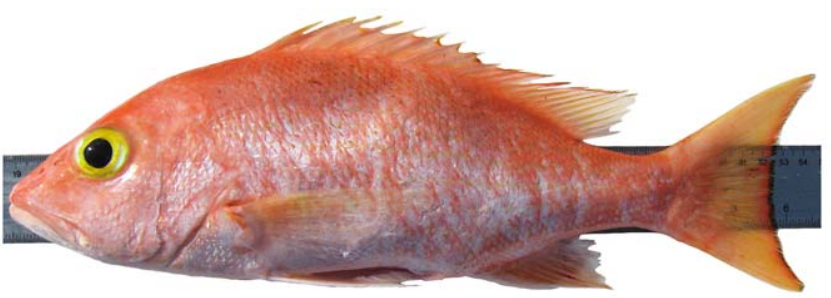

Figure 85. Lutjanus vivanus, $311 \mathrm{~mm} \mathrm{SL}$, photo by W Toller. doi:10.1371/journal.pone.0010676.g085

Ocyurus chrysurus (Bloch, 1791)-yellowtail snapper; UF, O, V

Pristipomoides aquilonaris (Goode \& Bean, 1896)-wenchman; USNM, F; Figure 86

Rhomboplites aurorubens (Cuvier, 1829) —vermilion snapper; USNM, F; Figure 87

Lobotidae-tripletails

Lobotes surinamensis (Bloch, 1790)—Atlantic tripletail; F; Figure 88

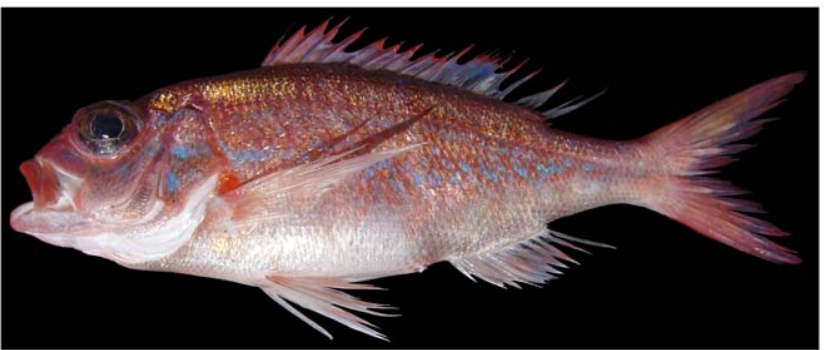

Figure 86. Pristipomoides aquilonaris, $263 \mathrm{~mm} \mathrm{SL}$, photo by W Toller.

doi:10.1371/journal.pone.0010676.g086 


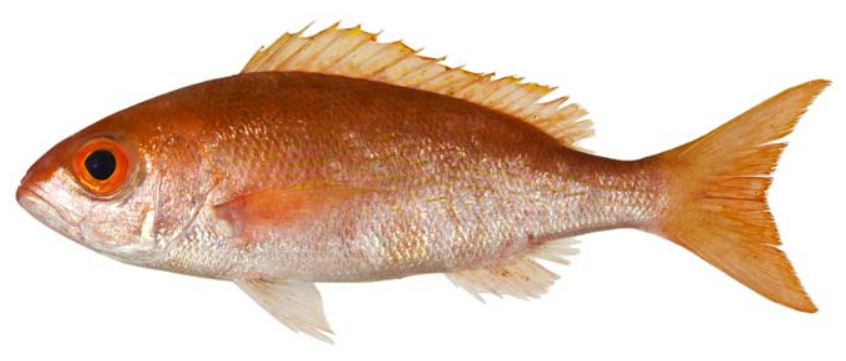

Figure 87. Rhomboplites aurorubens, $227.3 \mathrm{~mm} \mathrm{SL}$, photo by JT Williams.

doi:10.1371/journal.pone.0010676.g087

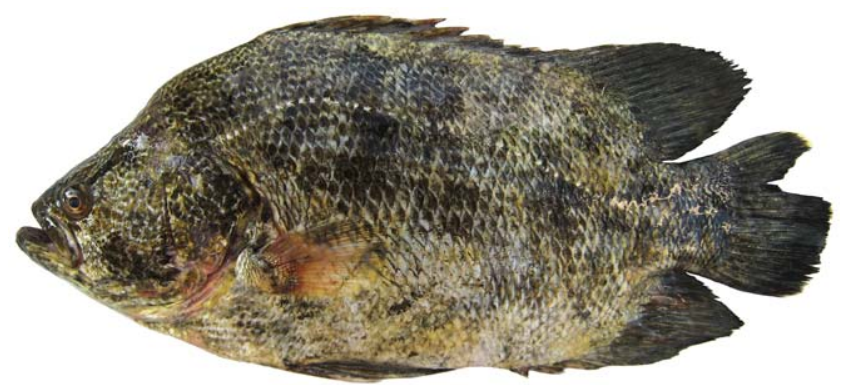

Figure 88. Lobotes surinamensis, $\mathbf{3 0 0} \mathbf{~ m m ~ S L , ~ p h o t o ~ b y ~ W ~ T o l l e r . ~}$ doi:10.1371/journal.pone.0010676.g088

Haemulidae-grunts

Haemulon album Cuvier, 1830 - margate; F; Figure 89 Haemulon aurolineatum Cuvier, 1830 - tomtate; F, O; Figure 90 Haemulon carbonarium Poey, 1860 - caesar grunt; F; Figure 91 Haemulon flavolineatum (Desmarest, 1823) — French grunt; $\mathbf{O}, \mathbf{V}$ Haemulon melanurum (Linnaeus, 1758) — cottonwick; UF, USNM, F, I, O, V; Figure 92

Haemulon plumierii (Lacepède, 1801) - white grunt; USNM, F, I, $\mathbf{O}, \mathbf{V}$; Figure 93

Haemulon striatum (Linnaeus, 1758)—striped grunt; F; Figure 94

Inermiidae-bonnetmouths

Inermia vittata Poey, 1860-boga; $\mathbf{O}$

\section{Sparidae-porgies}

Calamus calamus (Valenciennes, 1830)—saucereye porgy; V

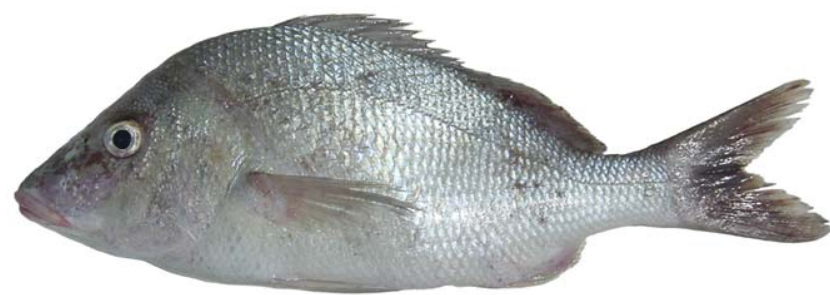

Figure 89. Haemulon album, 342 mm SL, photo by W Toller. doi:10.1371/journal.pone.0010676.g089

PLOS ONE I www.plosone.org

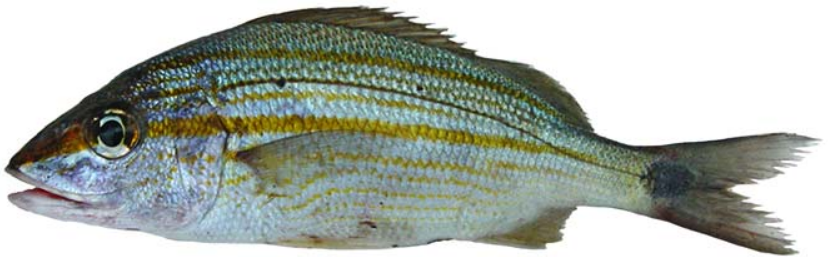

Figure 90. Haemulon aurolineatum, $175 \mathrm{~mm} \mathrm{SL}$, photo by W Toller.

doi:10.1371/journal.pone.0010676.g090

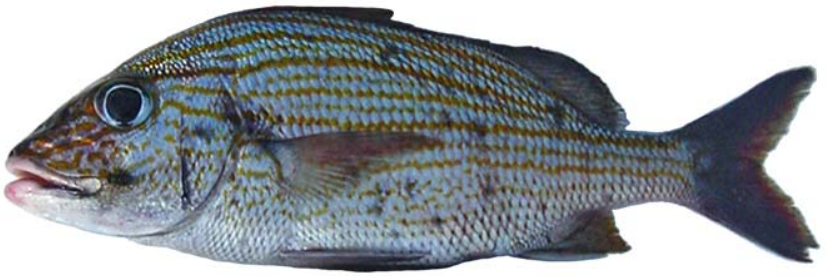

Figure 91. Haemulon carbonarium, approximately 200 mm SL, photo by W Toller.

doi:10.1371/journal.pone.0010676.g091

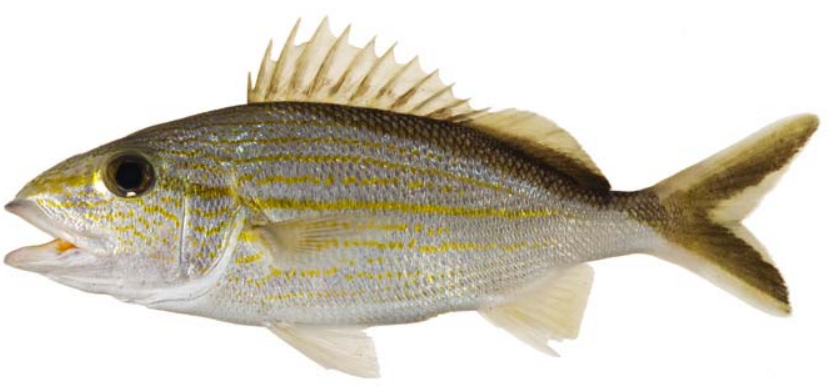

Figure 92. Haemulon melanurum, $213.8 \mathrm{~mm} \mathrm{SL}$, photo by JT Williams.

doi:10.1371/journal.pone.0010676.g092

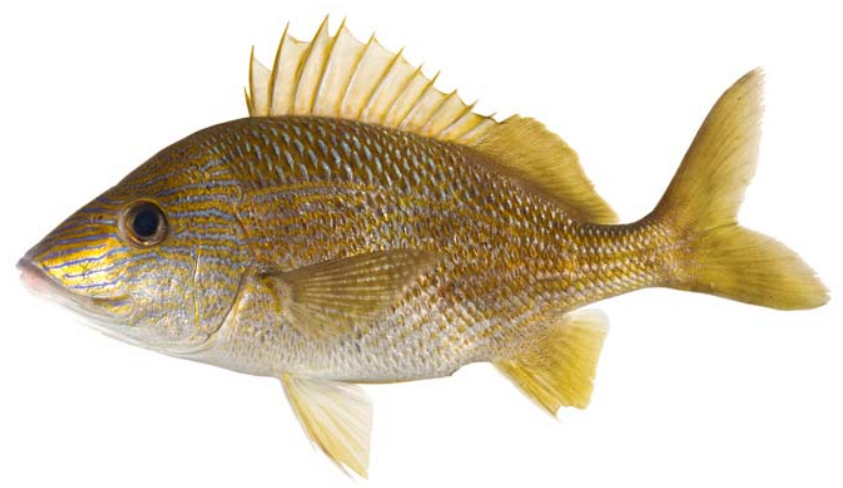

Figure 93. Haemulon plumierii, $244.7 \mathrm{~mm} \mathrm{SL}$, photo by JT Williams.

doi:10.1371/journal.pone.0010676.g093 


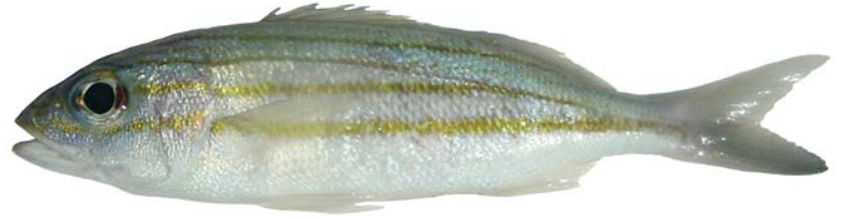

Figure 94. Haemulon striatum, $154 \mathrm{~mm} \mathrm{SL}$, photo by W Toller. doi:10.1371/journal.pone.0010676.g094

Sciaenidae-drums and croakers

Equetus punctatus (Bloch \& Schneider, 1801)—spotted drum; USNM, I; Figure 95

Pareques acuminatus (Bloch \& Schneider, 1801)-high-hat;

USNM, I; Figure 96

Mullidae-goatfishes

Mulloidichthys martinicus (Cuvier, 1829) - yellow goatfish; USNM,

F, I, O; Figure 97

Pseudupeneus maculatus (Bloch, 1793) — spotted goatfish; USNM,

I, O, V; Figure 98

Chaetodontidae-butterflyfishes

Chaetodon capistratus Linnaeus, 1758-foureye butterflyfish; USNM, I, O, V; Figure 99

Chaetodon ocellatus Bloch, 1787 -spotfin butterflyfish; USNM, F, $\mathbf{O}, \mathbf{V}$

Chaetodon sedentarius Poey, 1860 - reef butterflyfish; UF, O, V

Chaetodon striatus Linnaeus, 1758 - banded butterflyfish; USNM,

$\mathbf{I}, \mathbf{O}, \mathbf{V}$; Figure 100

Prognathodes aculeatus (Poey, 1860)-longsnout butterflyfish;

USNM, I, O, V; Figure 101

Pomacanthidae-angelfishes

Centropyge argi Woods \& Kanazawa, 1951 - cherubfish; USNM,

I, O, V; Figure 102

Holacanthus ciliaris (Linnaeus, 1758) — queen angelfish; USNM,

$\mathbf{F}, \mathbf{I}, \mathbf{O}, \mathbf{V}$

Holacanthus tricolor (Bloch, 1795) —rock beauty; USNM, I, O, V;

Figures 103, 104

Pomacanthus arcuatus (Linnaeus, 1758) - gray angelfish; V

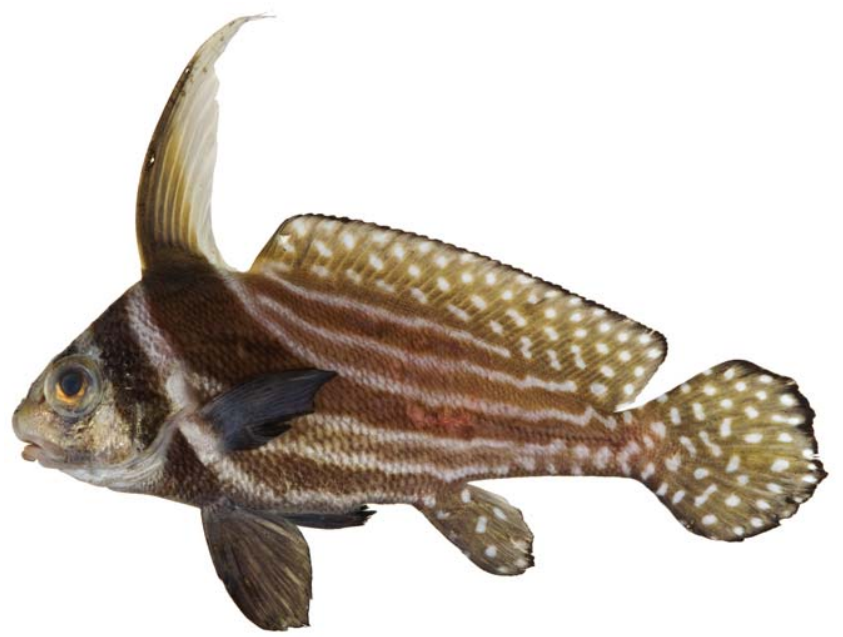

Figure 95. Equetus punctatus, $146.8 \mathrm{~mm} \mathrm{SL}$, photo by JT Williams.

doi:10.1371/journal.pone.0010676.g095

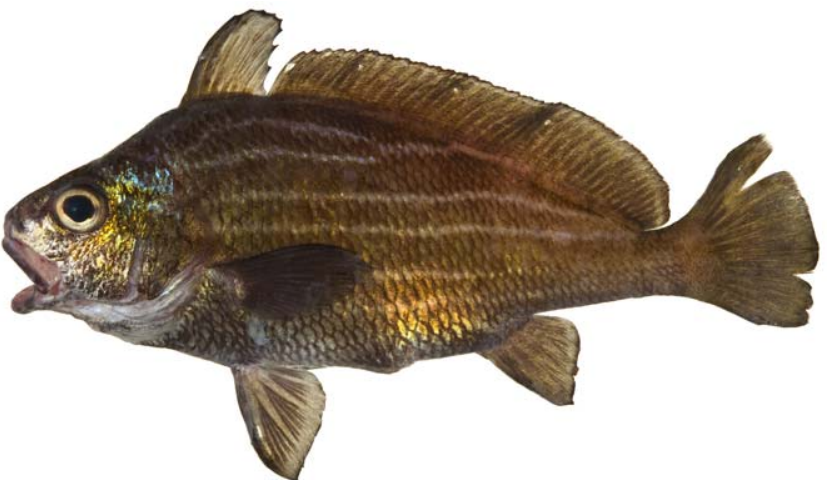

Figure 96. Pareques acuminatus, $150.0 \mathrm{~mm} \mathrm{SL}$, photo by JT Williams.

doi:10.1371/journal.pone.0010676.g096

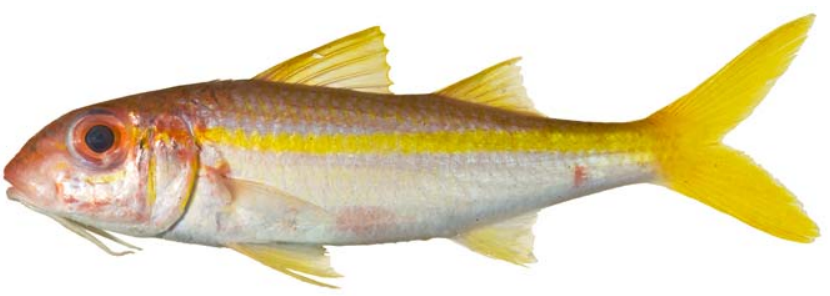

Figure 97. Mulloidichthys martinicus, $105 \mathrm{~mm} \mathrm{SL}$, photo by JT Williams.

doi:10.1371/journal.pone.0010676.g097

Pomacanthus paru (Bloch, 1787)—French angelfish; O, V

Kyphosidae-sea chub

Kyphosus incisor (Cuvier, 1831) - yellow chub; V

Kyphosus sectatrix (Linnaeus, 1766) - Bermuda chub; O, V

Cirrhitidae-hawkfishes

Amblycirrhitus pinos (Mowbray, 1927)—redspotted hawkfish; USNM, I, O; Figure 105

Pomacentridae-damselfishes

Chromis cyanea (Poey, 1860)—blue chromis; USNM, I, O, V; Figure 106

Chromis multilineata (Guichenot, 1853) - brown chromis; USNM,

I, O, V; Figure 107

Microspathodon chrysurus (Cuvier, 1830)—yellowtail damselfish; $\mathbf{O}, \mathbf{V}$

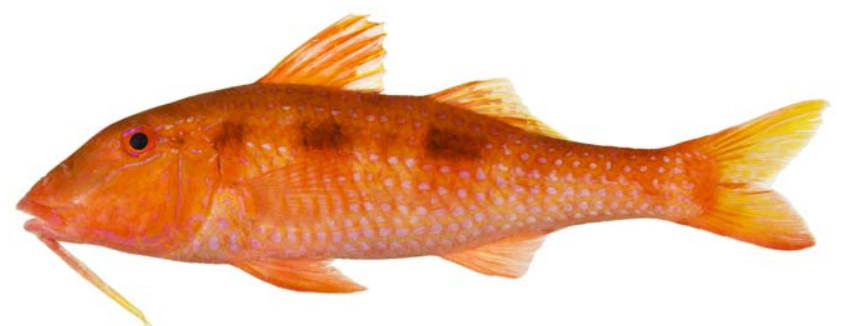

Figure 98. Pseudupeneus maculatus, $218.8 \mathrm{~mm}$ SL, photo by JT Williams.

doi:10.1371/journal.pone.0010676.g098 


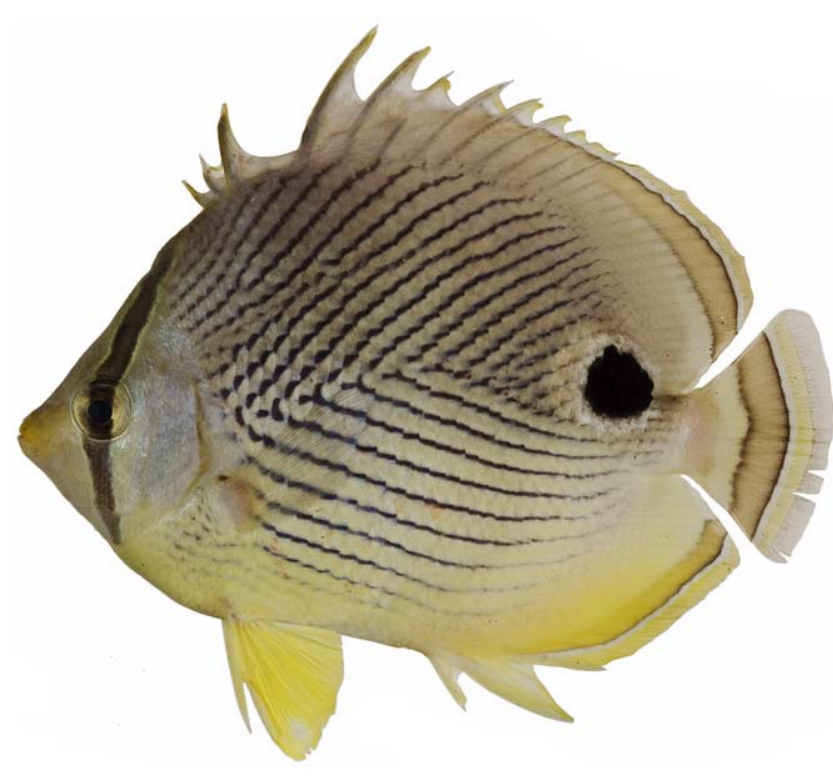

Figure 99. Chaetodon capistratus, $99.4 \mathrm{~mm} \mathrm{SL;} \mathrm{when} \mathrm{younger,}$ the specimen was apparently injured in the region of the fourth dorsal-fin spine (missing) and the area healed leaving a gap in the fin; photo by JT Williams.

doi:10.1371/journal.pone.0010676.g099

Stegastes adustus (Troschel, 1865) — dusky damselfish; O

Stegastes leucostictus (Müller \& Troschel, 1848) - beaugregory; O

Stegastes partitus (Poey, 1868)—bicolor damselfish; USNM, I, O, V; Figures 108, 109

This species has a variable color pattern throughout its range. Two color morphs were found at Saba Bank. Both morphs have a yellow pectoral fin and a reduced yellowish white area covering the caudal peduncle. One morph has a black caudal fin (Figure 108) and the other morph has a pale yellowish caudal fin with a dusky brown area in the middle of the upper and lower lobes (Figure 109).

Stegastes planifrons (Cuvier, 1830) - threespot damselfish; USNM, I, O; Figure 110

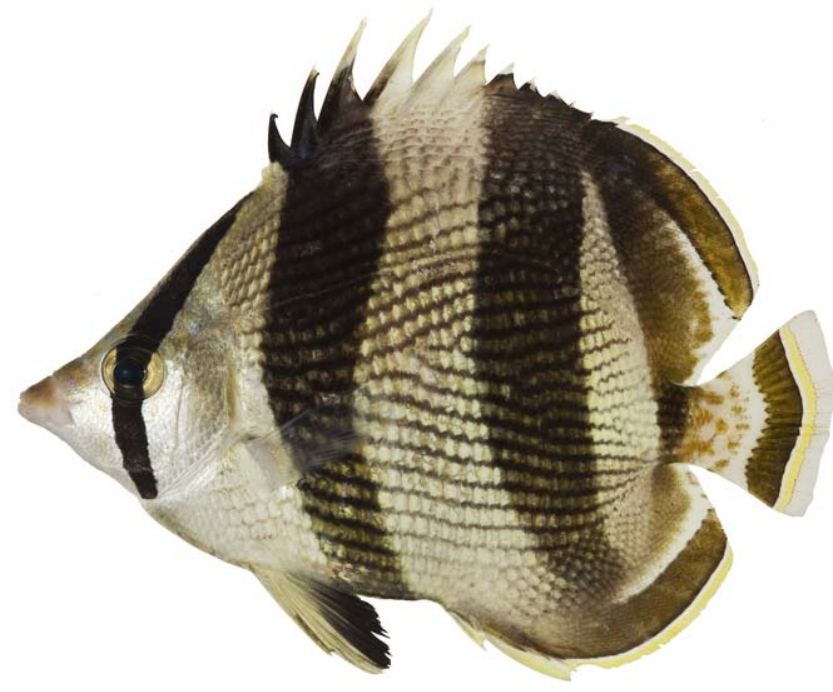

Figure 100. Chaetodon striatus, $111.3 \mathrm{~mm} \mathrm{SL}$, photo by JT Williams.

doi:10.1371/journal.pone.0010676.g100

PLOS ONE I www.plosone.org
Figure 101. Prognathodes aculeatus, $62.9 \mathrm{~mm} \mathrm{SL}$, photo by JT Williams.

doi:10.1371/journal.pone.0010676.g101

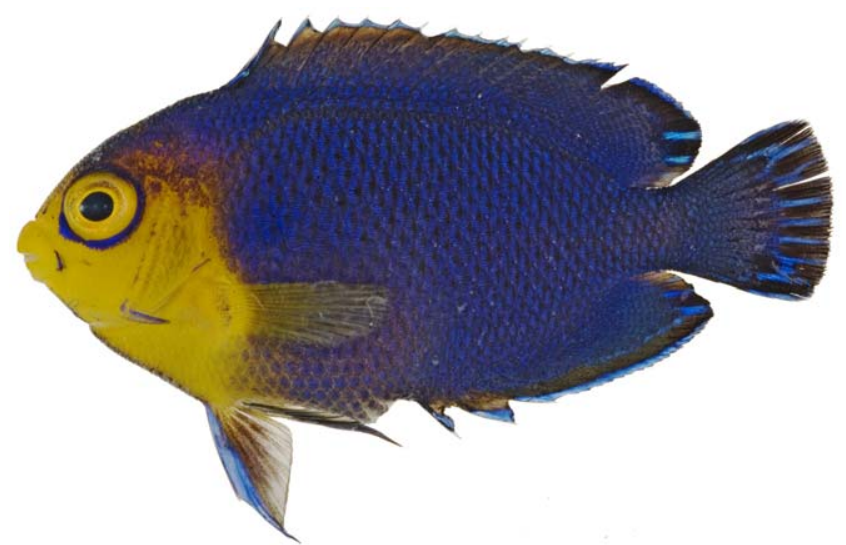

Figure 102. Centropyge argi, $33.8 \mathrm{~mm} \mathrm{SL}$, photo by JT Williams. doi:10.1371/journal.pone.0010676.g102

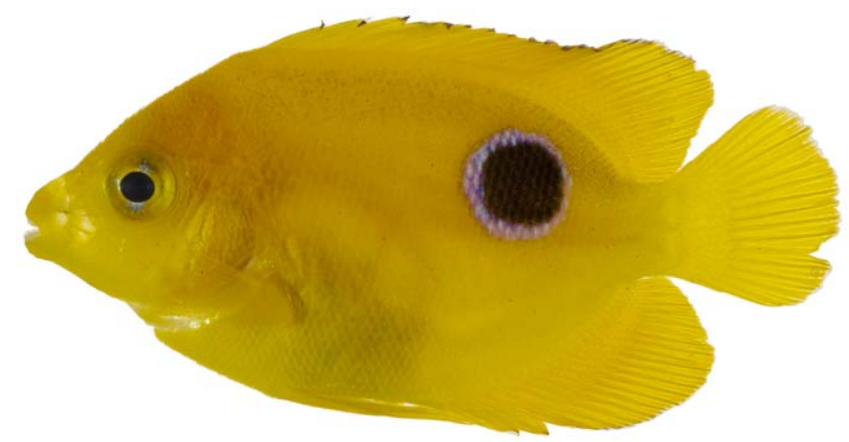

Figure 103. Holacanthus tricolor, $19.3 \mathrm{~mm} \mathrm{SL}$, juvenile color pattern, photo by JT Williams.

doi:10.1371/journal.pone.0010676.g103 


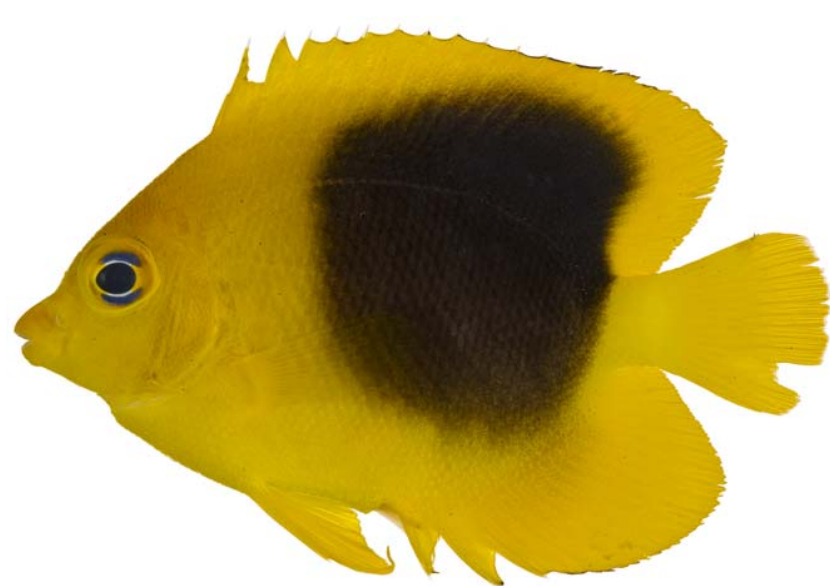

Figure 104. Holacanthus tricolor, $40.1 \mathrm{~mm} \mathrm{SL}$, intermediate color pattern, photo by JT Williams.

doi:10.1371/journal.pone.0010676.g104

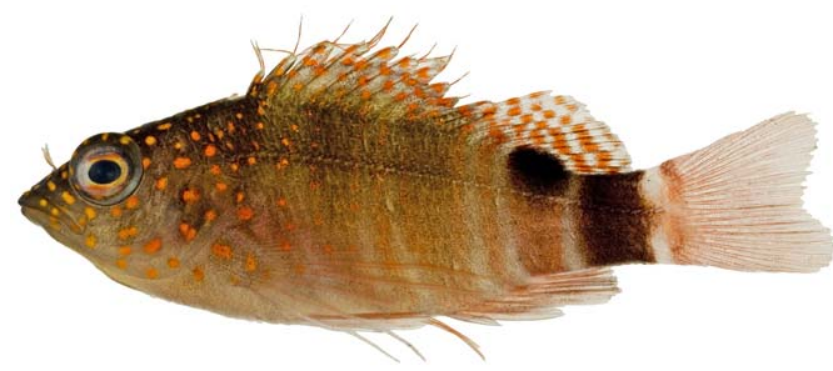

Figure 105. Amblycirrhitus pinos, $22.6 \mathrm{~mm} \mathrm{SL}$, photo by JT Williams.

doi:10.1371/journal.pone.0010676.g105

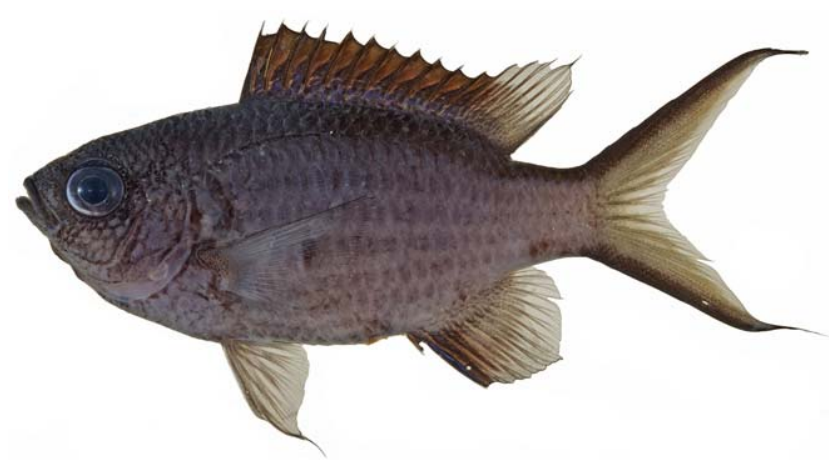

Figure 106. Chromis cyanea, $67.5 \mathrm{~mm} \mathrm{SL}$, iridescent blue colors on body faded immediately after death, photo by JT Williams. doi:10.1371/journal.pone.0010676.g106

PLOS ONE I www.plosone.org

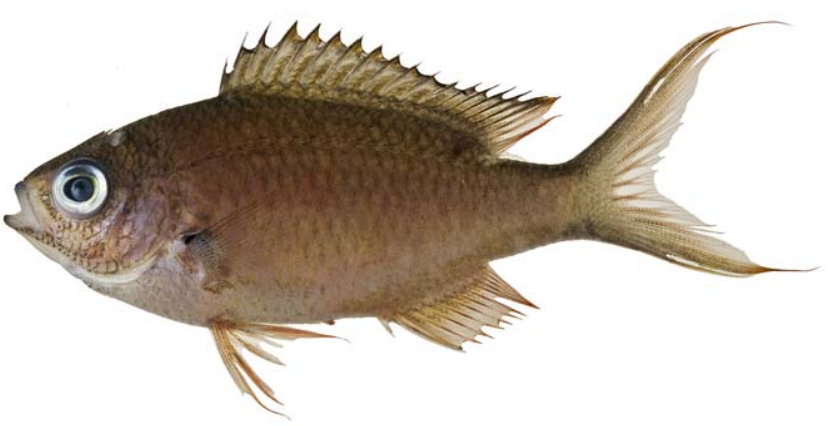

Figure 107. Chromis multilineata, $56.3 \mathrm{~mm} \mathrm{SL}$, photo by JT Williams.

doi:10.1371/journal.pone.0010676.g107

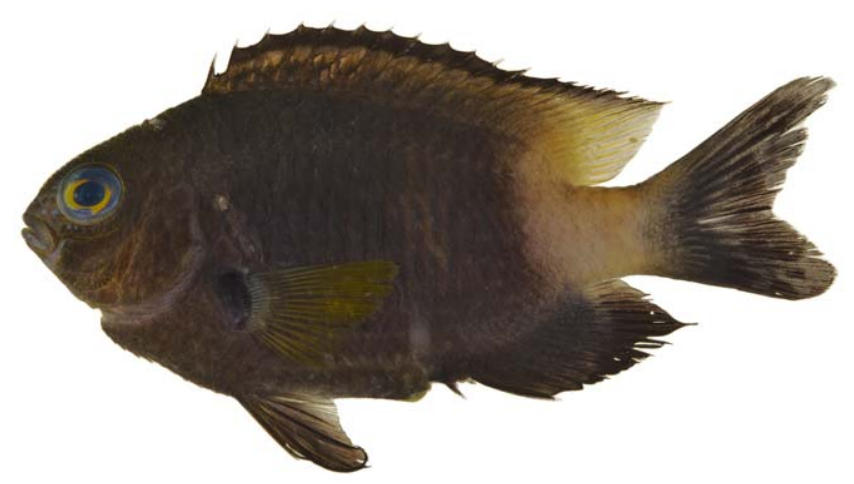

Figure 108. Stegastes partitus, $58.6 \mathrm{~mm} \mathrm{SL}$, black-tailed color morph, photo by JT Williams.

doi:10.1371/journal.pone.0010676.g108

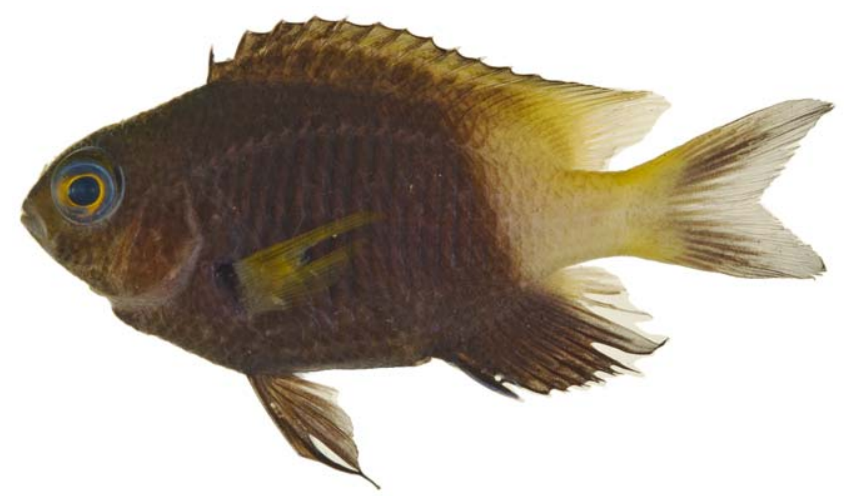

Figure 109. Stegastes partitus, $58.6 \mathrm{~mm} \mathrm{SL}$, yellow-tailed color morph, photo by JT Williams.

doi:10.1371/journal.pone.0010676.g109 


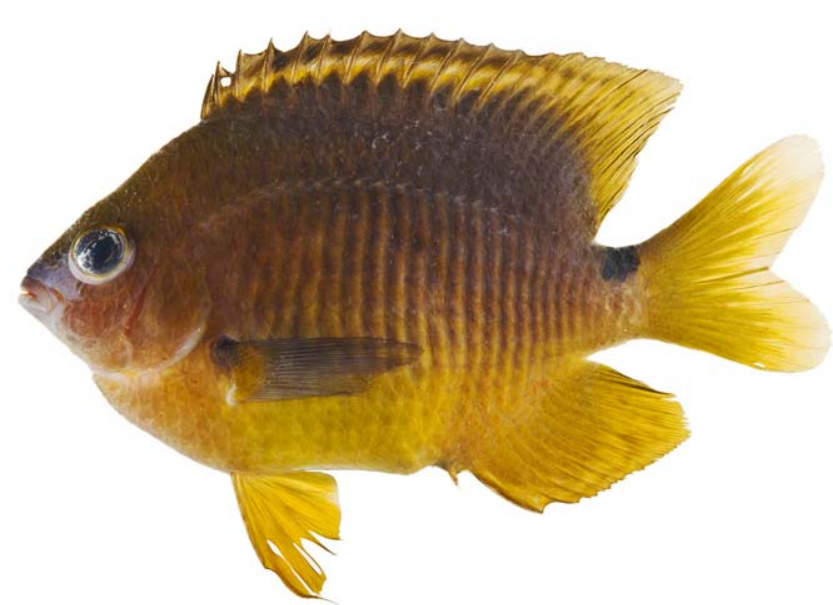

Figure 110. Stegastes planifrons, $82.7 \mathrm{~mm} \mathrm{SL}$, photo by JT Williams.

doi:10.1371/journal.pone.0010676.g110

\section{Labridae-wrasses}

Bodianus rufus (Linnaeus, 1758)-Spanish hogfish; O, V

Clepticus parrae (Bloch \& Schneider, 1801)-creole wrasse;

USNM, I, O, V; Figure 111

Doratonotus megalepis Günther, 1862 - dwarf wrasse; USNM, I, V; Figure 112

Halichoeres bivittatus (Bloch, 1791) - slippery dick; USNM, I, O,

V; Figures 113, 114

Halichoeres cyanocephalus (Bloch, 1791)-yellowcheek wrasse; $\mathbf{O}, \mathbf{V}$

Halichoeres garnoti (Valenciennes, 1839) - yellowhead wrasse; UF,

USNM, I, O, V; Figures 115, 116, 117

These figures show portions of the transitional color phases as individuals transform from juveniles (Figure 115) into initial phase females (Figure 116) and finally into terminal phase males (Figure 117).

Halichoeres maculipinna (Müller \& Troschel, 1848)—clown wrasse;

\section{USNM, I, $\mathbf{O}$}

Halichoeres pictus (Poey, 1860) - rainbow wrasse; USNM, I, O;

Figures 118, 119

Halichoeres poeyi (Steindachner, 1867)-blackear wrasse; UF,

USNM, I, O, V; Figure 120

Halichoeres radiatus (Linnaeus, 1758)-puddingwife; $\mathbf{O}, \mathbf{V}$

Lachnolaimus maximus (Walbaum, 1792)-hogfish; USNM

Thalassoma bifasciatum (Bloch, 1791)-bluehead; USNM, I, O,

V; Figures 121, 122

Xyrichtys splendens Castelnau, 1855 - green razorfish; USNM, I, O; Figures 123, 124, 125, 126

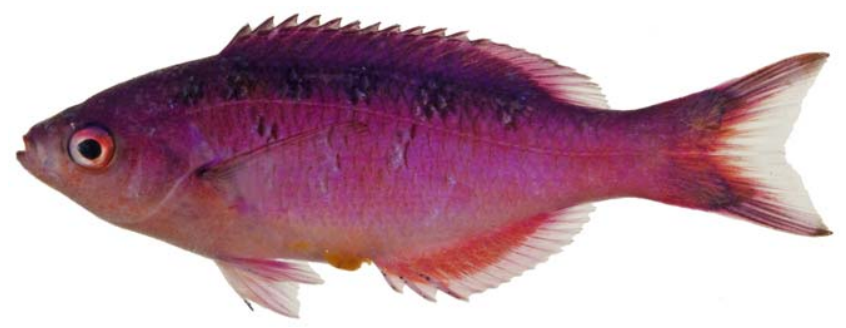

Figure 111. Clepticus parrae, $59.8 \mathrm{~mm} \mathrm{SL}$, photo by JT Williams. doi:10.1371/journal.pone.0010676.g111

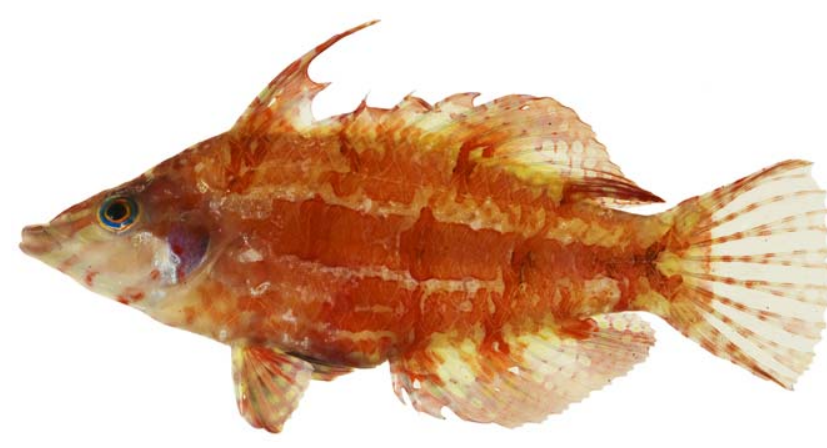

Figure 112. Doratonotus megalepis, $44.1 \mathrm{~mm} \mathrm{SL}$, photo by JT Williams.

doi:10.1371/journal.pone.0010676.g112

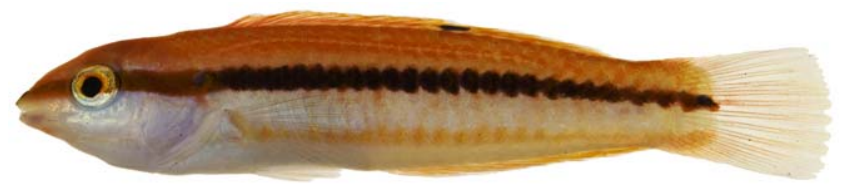

Figure 113. Halichoeres bivittatus, $37.9 \mathrm{~mm} \mathrm{SL}$, juvenile/initial color phase, showing the black spot in the dorsal fin and a somewhat unusual orangish anal fin, photo by JT Williams. doi:10.1371/journal.pone.0010676.g113

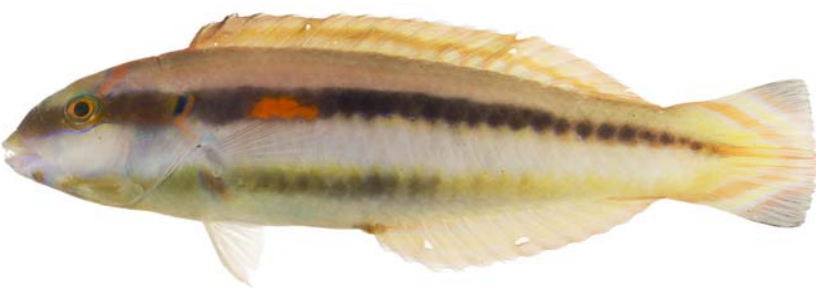

Figure 114. Halichoeres bivittatus, $103.0 \mathrm{~mm} \mathrm{SL}$, terminal male color phase, with a distinctive red blotch on the side of the body above the pectoral fin, photo by JT Williams.

doi:10.1371/journal.pone.0010676.g114

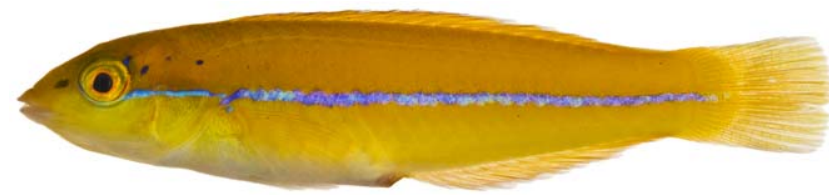

Figure 115. Halichoeres garnoti, $46.4 \mathrm{~mm} \mathrm{SL}$, juvenile color phase, showing the characteristic blue stripe on a yellow body, photo by JT Williams.

doi:10.1371/journal.pone.0010676.g115 


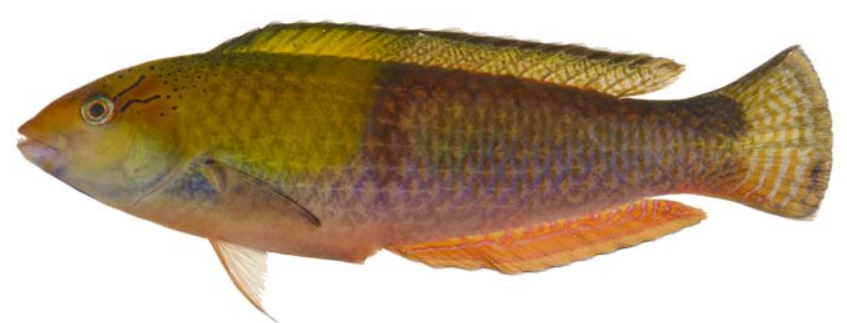

Figure 116. Halichoeres garnoti, $118.1 \mathrm{~mm} \mathrm{SL}$, initial/terminal color phase, this specimen has almost completed the transition from the female initial phase into a terminal male, photo by JT Williams.

doi:10.1371/journal.pone.0010676.g116

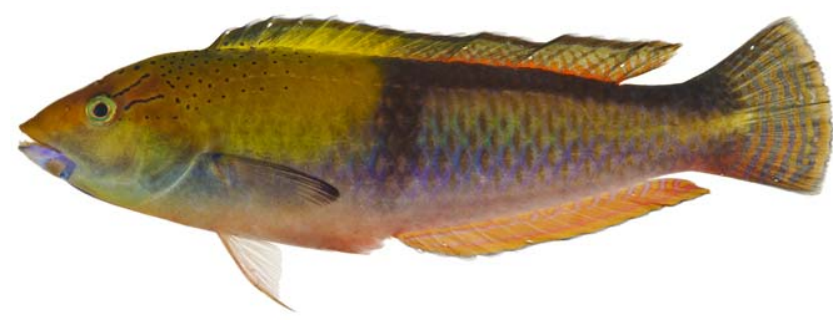

Figure 117. Halichoeres garnoti, $125.3 \mathrm{~mm} \mathrm{SL}$, terminal male color phase, this specimen has completed the transition from the female initial phase into a terminal male and shows the characteristic black bar and dark area over the caudal peduncle, photo by JT Williams. doi:10.1371/journal.pone.0010676.g117

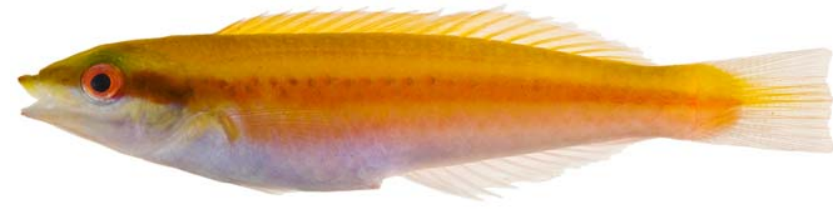

Figure 118. Halichoeres pictus, $70.7 \mathrm{~mm}$ SL, juvenile color phase, photo by JT Williams.

doi:10.1371/journal.pone.0010676.g118

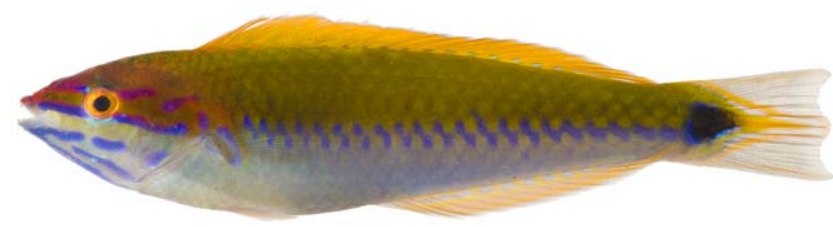

Figure 119. Halichoeres pictus, $85.0 \mathrm{~mm} \mathrm{SL}$, terminal male color phase, photo by JT Williams.

doi:10.1371/journal.pone.0010676.g119

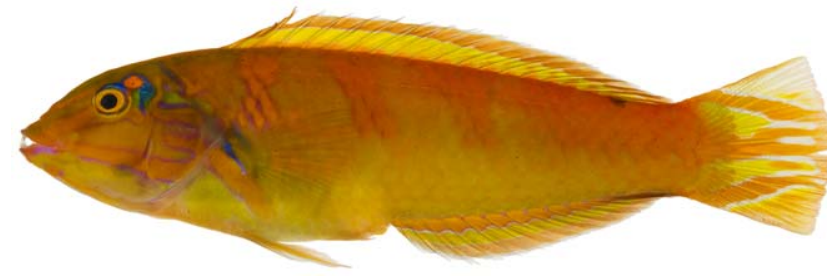

Figure 120. Halichoeres poeyi, $87.9 \mathrm{~mm} \mathrm{SL}$, terminal male color phase, photo by JT Williams.

doi:10.1371/journal.pone.0010676.g120

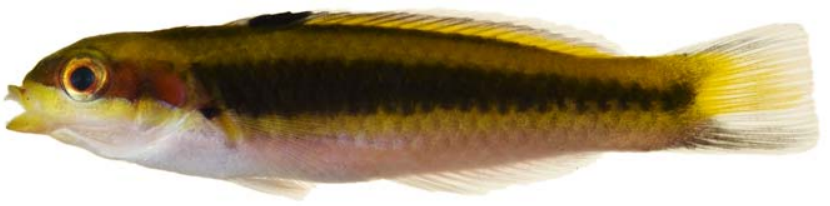

Figure 121. Thalassoma bifasciatum, $39.3 \mathrm{~mm} \mathrm{SL}$, juvenile/initial color phase, photo by JT Williams.

doi:10.1371/journal.pone.0010676.g121

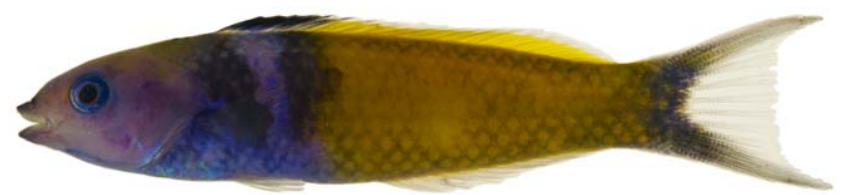

Figure 122. Thalassoma bifasciatum, $63.5 \mathrm{~mm} \mathrm{SL}$, terminal male color phase, photo by JT Williams. doi:10.1371/journal.pone.0010676.g122

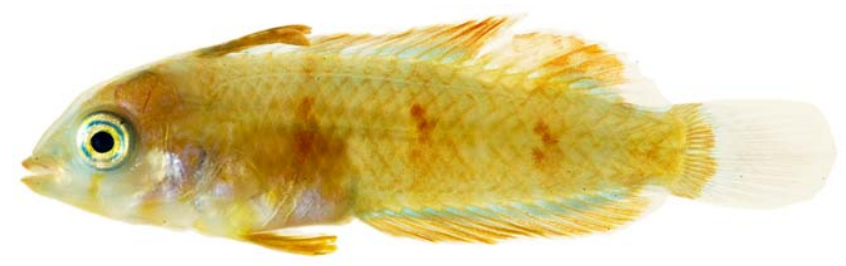

Figure 123. Xyrichtys splendens, $15.1 \mathrm{~mm} \mathrm{SL}$, young juvenile color phase, photo by JT Williams. doi:10.1371/journal.pone.0010676.g123

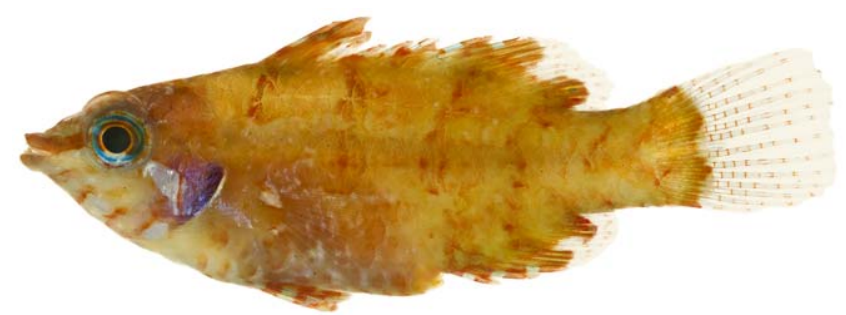

Figure 124. Xyrichtys splendens, $22.1 \mathrm{~mm} \mathrm{SL}$, juvenile color phase, photo by JT Williams.

doi:10.1371/journal.pone.0010676.g124 


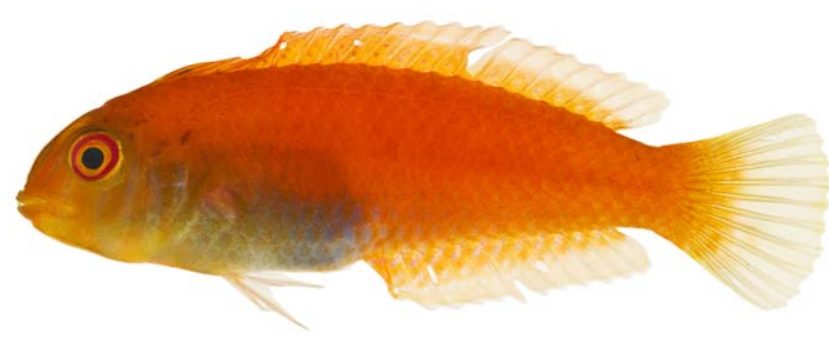

Figure 125. Xyrichtys splendens, $\mathbf{5 2 . 4} \mathbf{~ m m ~ S L}$, initial color phase of female, photo by JT Williams.

doi:10.1371/journal.pone.0010676.g125

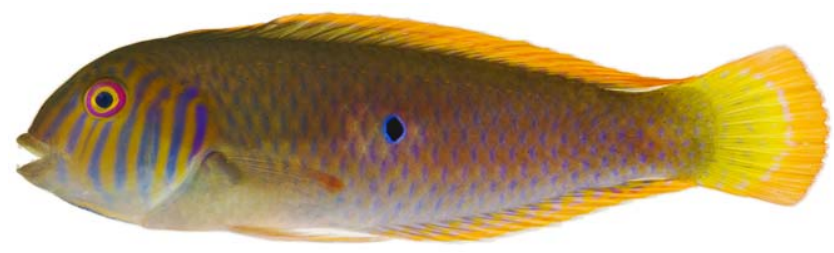

Figure 126. Xyrichtys splendens, $72.5 \mathrm{~mm} \mathrm{SL}$, terminal male color phase, photo by JT Williams.

doi:10.1371/journal.pone.0010676.g126

\section{Scaridae-parrotfishes}

Cryptotomus roseus Cope, 1871 - bluelip parrotfish; UF, USNM,

I, O; Figure 127

Scarus coelestinus Valenciennes, 1840 - midnight parrotfish; V Scarus guacamaia Cuvier, 1829 rainbow parrotfish; F; Figure 128 Scarus iseri (Bloch, 1789) — striped parrotfish; USNM, O, V

Scarus taeniopterus Desmarest, 1831 - princess parrotfish; USNM,

I, O, V; Figure 129, 130

Scarus vetula Bloch \& Schneider, 1801 - queen parrotfish; $\mathbf{O}$

Sparisoma atomarium (Poey, 1861)-greenblotch parrotfish;

USNM, I, O; Figure 131

Sparisoma aurofrenatum (Valenciennes, 1840) — redband parrotfish;

USNM, I, O, V; Figures 132, 133, 134

Sparisoma chrysopterum (Bloch \& Schneider, 1801) - redtail parrotfish; USNM, I, O; Figure 135

Sparisoma radians (Valenciennes, 1840)—bucktooth parrotfish;

USNM, I, O, V; Figure 136

Sparisoma viride (Bonnaterre, 1788)—stoplight parrotfish;

USNM, I, O, V; Figure 137

Tripterygiidae-triplefins

Enneanectes altivelis Rosenblatt, 1960 - lofty triplefin; USNM, I;

Figure 138

Enneanectes atrorus Rosenblatt, 1960 - redeye triplefin; USNM, I

Enneanectes jordani (Evermann and Marsh, 1899) - mimic triplefin; USNM, I

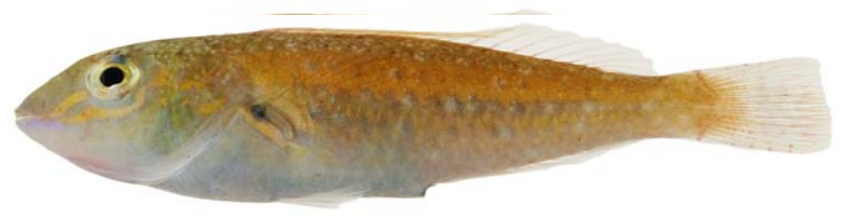

Figure 127. Cryptotomus roseus, $61.5 \mathrm{~mm} \mathrm{SL}$, terminal male color phase, photo by JT Williams.

doi:10.1371/journal.pone.0010676.g127

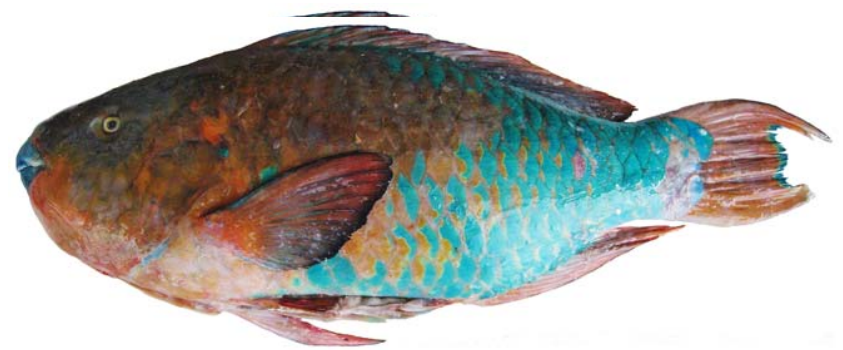

Figure 128. Scarus guacamaia, $647 \mathrm{~mm} \mathrm{SL}$, terminal male color phase, photo by $\mathbf{W}$ Toller.

doi:10.1371/journal.pone.0010676.g128

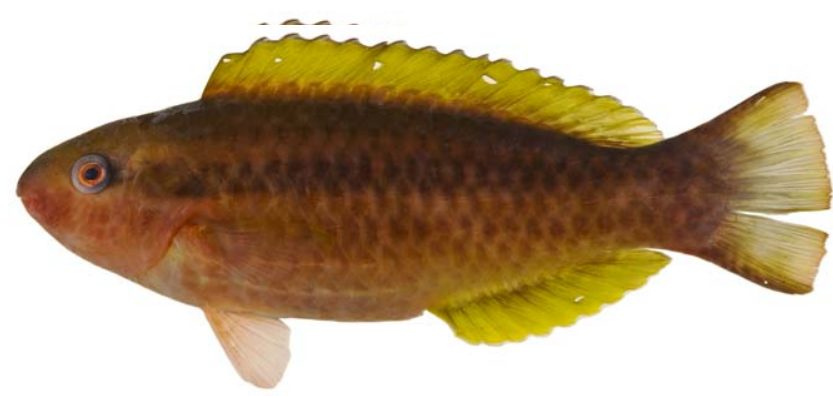

Figure 129. Scarus taeniopterus, $174.9 \mathrm{~mm} \mathrm{SL}$, initial female color phase, photo by JT Williams. doi:10.1371/journal.pone.0010676.g129

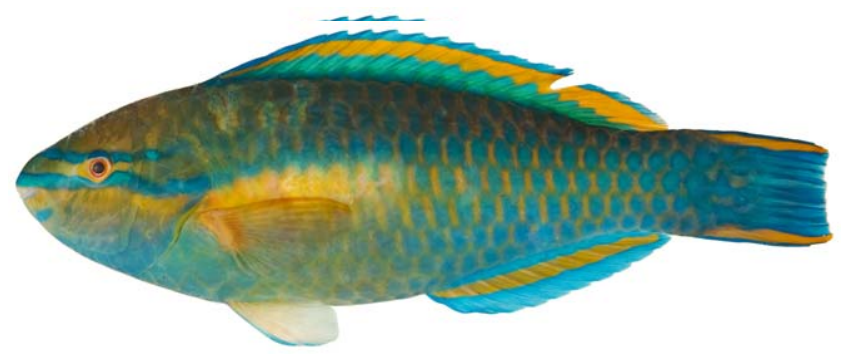

Figure 130. Scarus taeniopterus, $231.7 \mathrm{~mm} \mathrm{SL}$, terminal male color phase, photo by JT Williams. doi:10.1371/journal.pone.0010676.g130

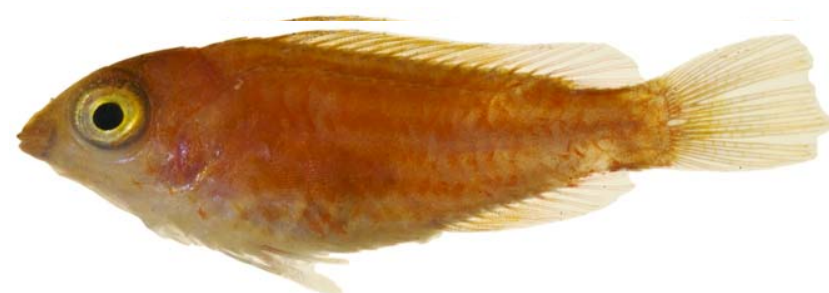

Figure 131. Sparisoma atomarium, $13.7 \mathrm{~mm} \mathrm{SL}$, juvenile color phase, photo by JT Williams.

doi:10.1371/journal.pone.0010676.g131 


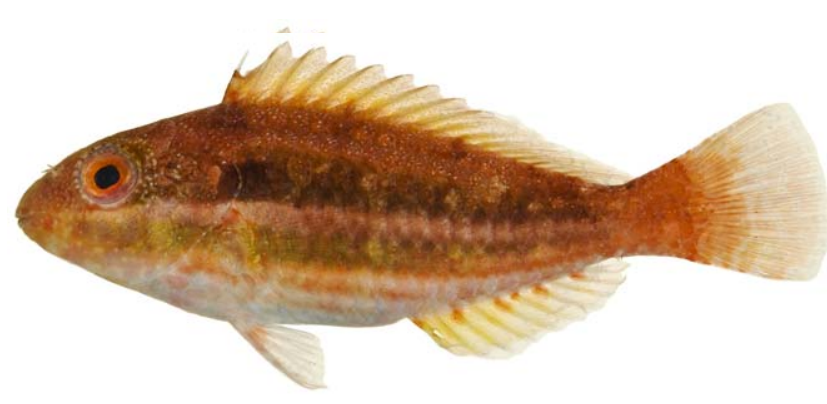

Figure 132. Sparisoma aurofrenatum, $53.8 \mathrm{~mm} \mathrm{SL}$, juvenile color phase, photo by JT Williams.

doi:10.1371/journal.pone.0010676.g132

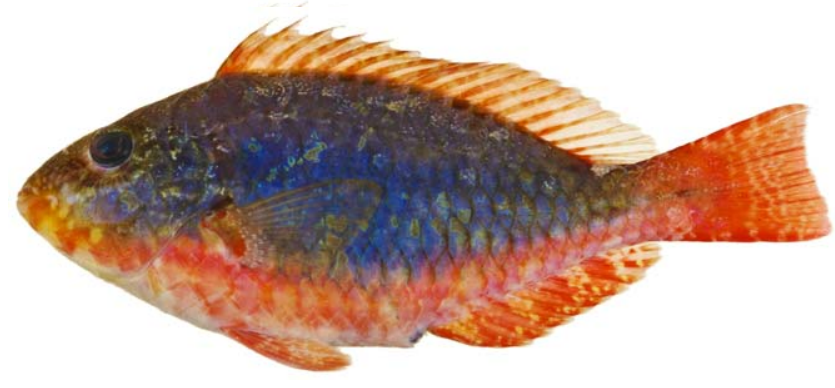

Figure 133. Sparisoma aurofrenatum, $164.0 \mathrm{~mm} \mathrm{SL}$, initial female color phase, photo by JT Williams.

doi:10.1371/journal.pone.0010676.g133

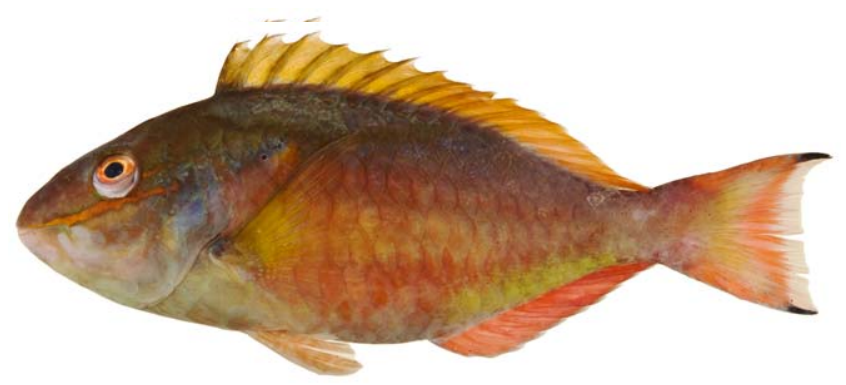

Figure 134. Sparisoma aurofrenatum, $164 \mathrm{~mm} \mathrm{SL}$, terminal male color phase, photo by JT Williams. doi:10.1371/journal.pone.0010676.g134

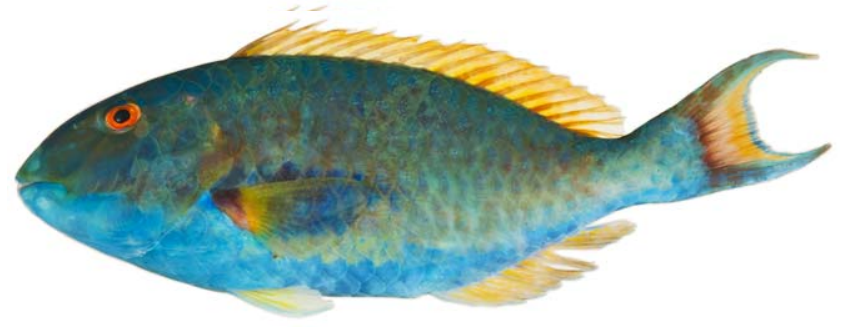

Figure 135. Sparisoma chrysopterum, $274 \mathrm{~mm} \mathrm{SL}$, terminal male color phase, photo by JT Williams. doi:10.1371/journal.pone.0010676.g135

PLOS ONE I www.plosone.org

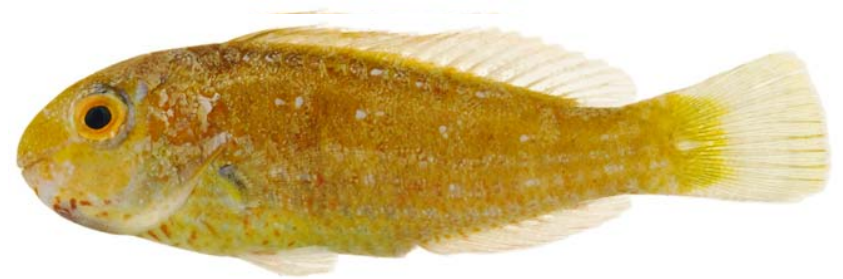

Figure 136. Sparisoma radians, $51.6 \mathrm{~mm} \mathrm{SL}$, juvenile color phase, photo by JT Williams.

doi:10.1371/journal.pone.0010676.g136

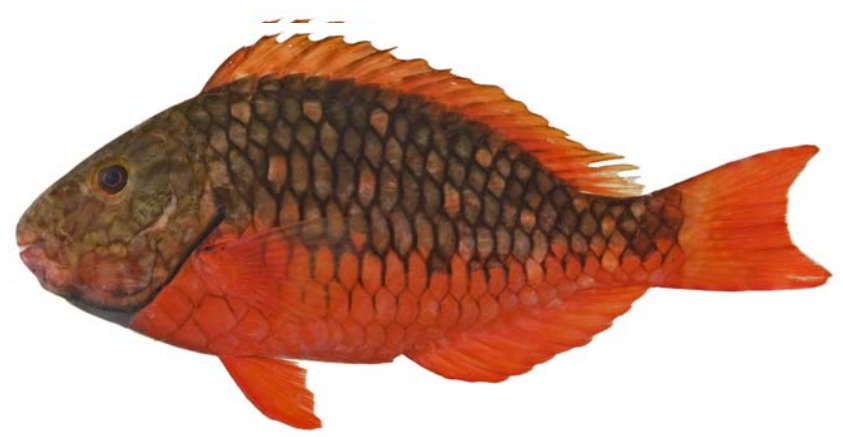

Figure 137. Sparisoma viride, $222.9 \mathrm{~mm} \mathrm{SL}$, initial female color phase, photo by JT Williams.

doi:10.1371/journal.pone.0010676.g137

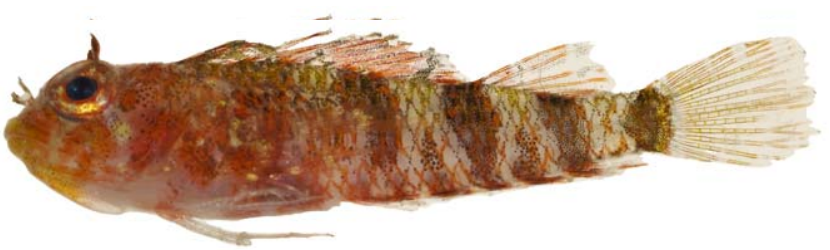

Figure 138. Enneanectes altivelis, $18.3 \mathrm{~mm} \mathrm{SL}$, photo by JT Williams.

doi:10.1371/journal.pone.0010676.g138

Dactyloscopidae-sand stargazers

Dactyloscopus tridigitatus Gill, 1859 - sand stargazer; USNM, I; Figures 139, 140

Gillellus uranidea Böhlke, 1968 - warteye stargazer; USNM, I; Figure 141

Platygillellus rubrocinctus (Longley, 1934) - saddle stargazer; USNM, I; Figures 142, 143, 144

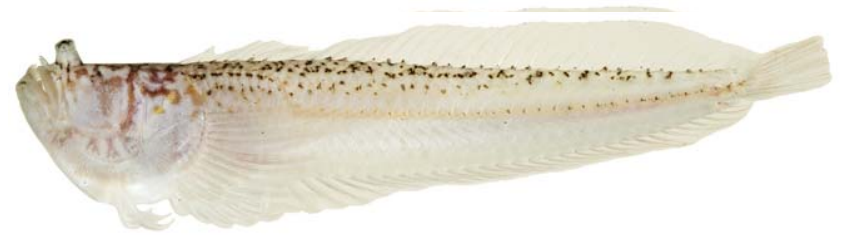

Figure 139. Dactyloscopus tridigitatus, $50.8 \mathrm{~mm} \mathrm{SL}$, photo by JT Williams.

doi:10.1371/journal.pone.0010676.g139 


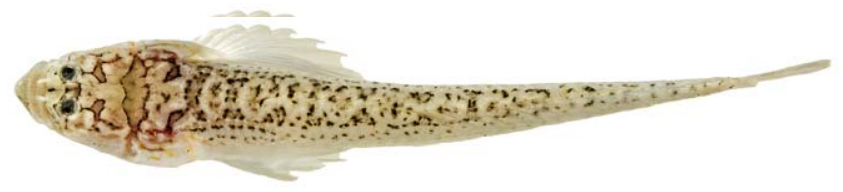

Figure 140. Dactyloscopus tridigitatus, $50.8 \mathrm{~mm} \mathrm{SL}$, dorsal view, photo by JT Williams.

doi:10.1371/journal.pone.0010676.g140

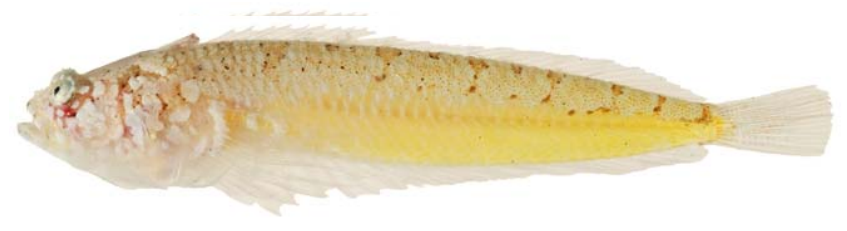

Figure 141. Gillellus uranidea, $27.7 \mathrm{~mm} \mathrm{SL}$, photo by JT Williams. doi:10.1371/journal.pone.0010676.g141

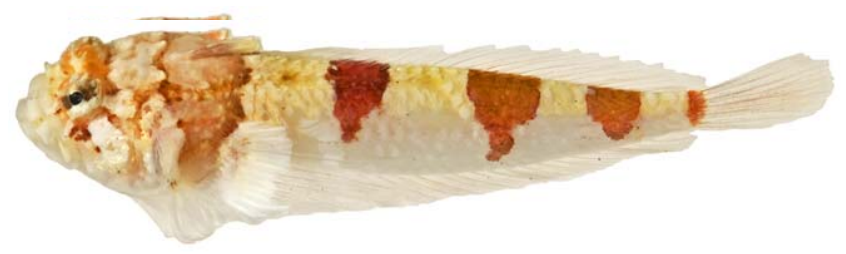

Figure 142. Platygillellus rubrocinctus, $24.1 \mathrm{~mm} \mathrm{SL}$, adult, photo by JT Williams.

doi:10.1371/journal.pone.0010676.g142

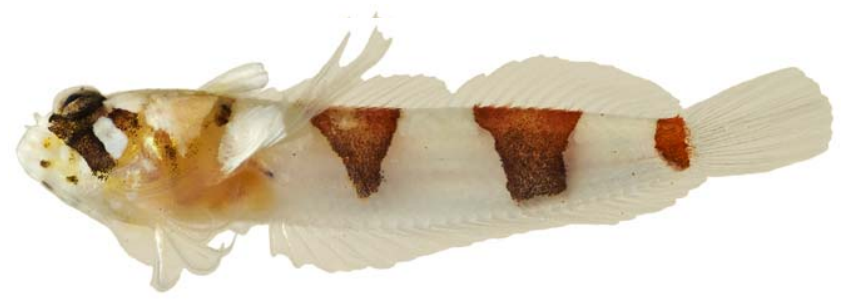

Figure 143. Platygillellus rubrocinctus, $12.1 \mathrm{~mm} \mathrm{SL}$, lateral view of juvenile, photo by JT Williams.

doi:10.1371/journal.pone.0010676.g143

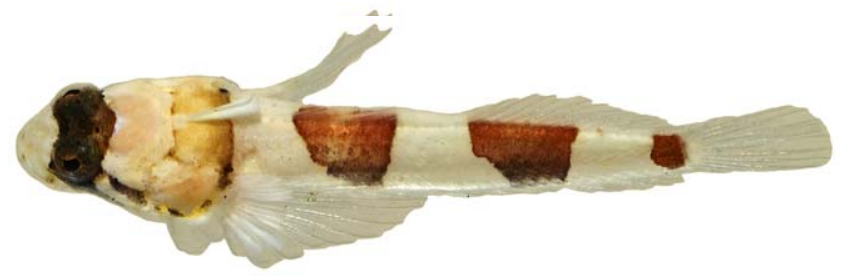

Figure 144. Platygillellus rubrocinctus, $12.1 \mathrm{~mm} \mathrm{SL}$, dorsal view of juvenile, photo by JT Williams.

doi:10.1371/journal.pone.0010676.g144
Blenniidae-combtooth blennies

Parablennius marmoreus (Poey, 1876) — seaweed blenny; USNM, I

Labrisomidae—scaly blennies

Labrisomus gobio (Valenciennes, 1836)-palehead blenny; USNM, I; Figure 145

Labrisomus haitiensis Beebe \&Tee-Van, 1918-longfin blenny;

USNM, I; Figure 146

Malacoctenus boehlkei Springer, 1959 - diamond blenny; USNM,

I; Figure 147

Paraclinus grandicomis (Rosen, 1911)-horned blenny; UF,

USNM, I; Figure 148

Starksia atlantica Longley, 1934 - smootheye blenny; USNM, I; Figures 149, 150

The Saba Bank population may be a distinct species in the species complex currently referred to as Starksia atlantica. This complex requires additional taxonomic study.

Starksia cf lepicoelia Böhlke \& Springer, 1961-blackcheek blenny; USNM, I; Figures 151, 152

Females (Figure 151) lack the black spot on the cheek that is characteristic of mature males (Figure 152). The Saba Bank population may be a distinct species in the species complex currently referred to as Starksia lepicoelia. This complex requires additional taxonomic study.

Starksia melasma Williams \& Mounts, 2003-black spot blenny; USNM, I

Starksia nanodes Böhlke \& Springer, 1961-dwarf blenny; USNM, I; Figure 153

The Saba Bank population may be a distinct species in the species complex currently referred to as Starksia nanodes. This complex requires additional taxonomic study.

Chaenopsidae-tube blennies

Acanthemblemaria aspera (Longley, 1927) — roughhead blenny; USNM, I; Figures 154, 155, 156

Three different color patterns were observed at Saba Bank: juvenile/female (Figure 154), adult male (Figure 155), and a distinctive yellow, probably female, color morph (Figure 156). The adult male and the yellow morph were taken together at the same collecting station.

Emblemaria pandionis Evermann \& Marsh, 1900 - sailfin blenny; USNM, I; Figure 157

Emblemariopsis cf signifer (Ginsburg, 1942)-flagfin blenny; USNM, I; Figure 158

The signifer species complex ranges from Brazil throughout the Caribbean and includes a number of undescribed species in the Caribbean region. Additional taxonomic study is required to resolve the taxa.

Gobiesocidae-clingfishes

Acyrtus artius Briggs, 1955-papillate clingfish; USNM, I; Figures 159, 160, 161

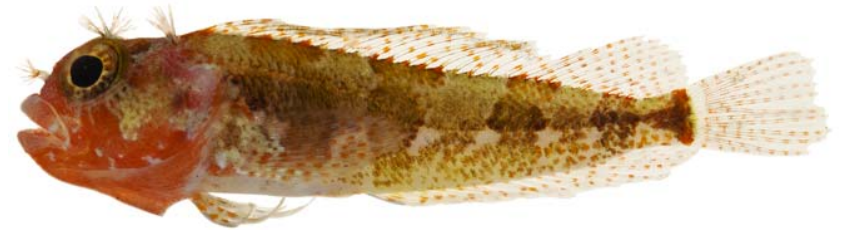

Figure 145. Labrisomus gobio, $36.2 \mathrm{~mm} \mathrm{SL}$, photo by JT Williams.

doi:10.1371/journal.pone.0010676.g145 


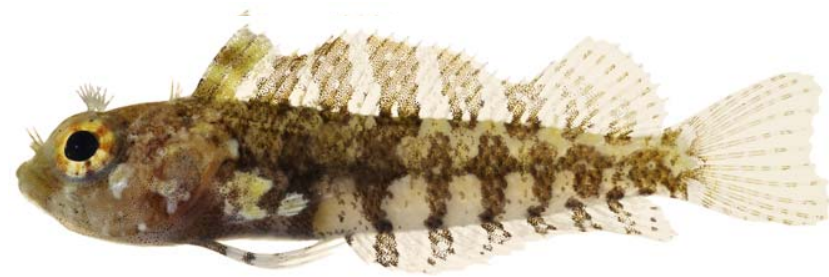

Figure 146. Labrisomus haitiensis, $18.8 \mathrm{~mm} \mathrm{SL}$, photo by JT Williams.

doi:10.1371/journal.pone.0010676.g146

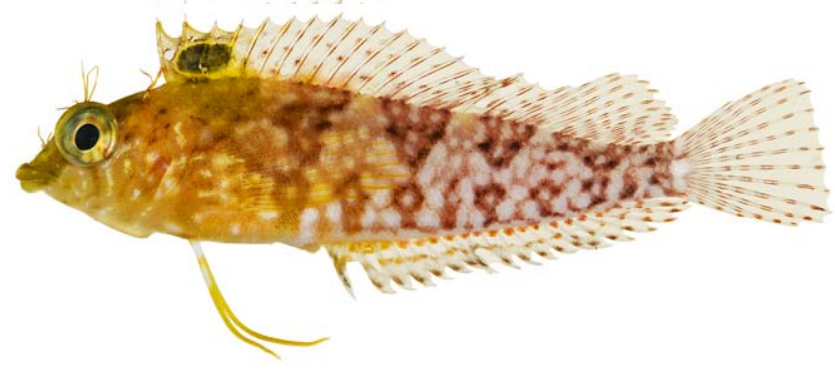

Figure 147. Malacoctenus boehlkei, $36.3 \mathrm{~mm} \mathrm{SL}$, photo by JT Williams.

doi:10.1371/journal.pone.0010676.g147

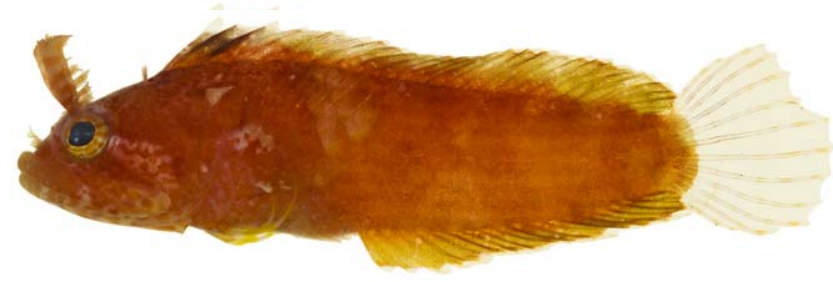

Figure 148. Paraclinus grandicomis, $27.1 \mathrm{~mm} \mathrm{SL}$, photo by JT Williams.

doi:10.1371/journal.pone.0010676.g148

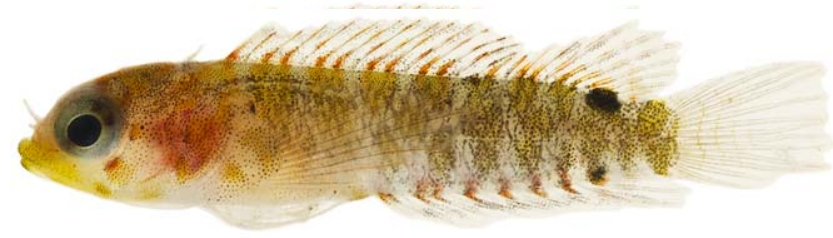

Figure 149. Starksia atlantica, $9.5 \mathrm{~mm} \mathrm{SL}$, juvenile, photo by JT Williams.

doi:10.1371/journal.pone.0010676.g149

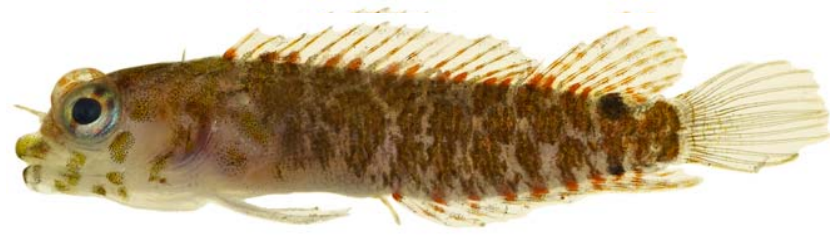

Figure 150. Starksia atlantica, $15.1 \mathrm{~mm} \mathrm{SL}$, adult male, photo by JT Williams.

doi:10.1371/journal.pone.0010676.g150

PLOS ONE I www.plosone.org

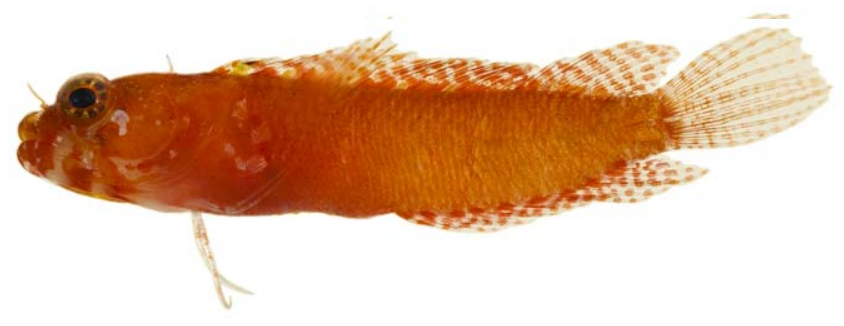

Figure 151. Starksia lepicoelia, $20.2 \mathrm{~mm} \mathrm{SL}$, adult female, photo by JT Williams.

doi:10.1371/journal.pone.0010676.g151

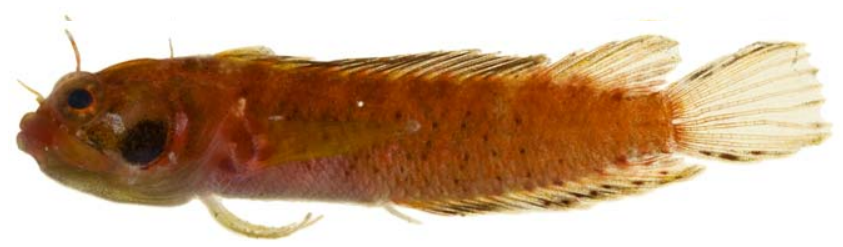

Figure 152. Starksia lepicoelia, $19.8 \mathrm{~mm} \mathrm{SL}$, adult male, photo by JT Williams.

doi:10.1371/journal.pone.0010676.g152

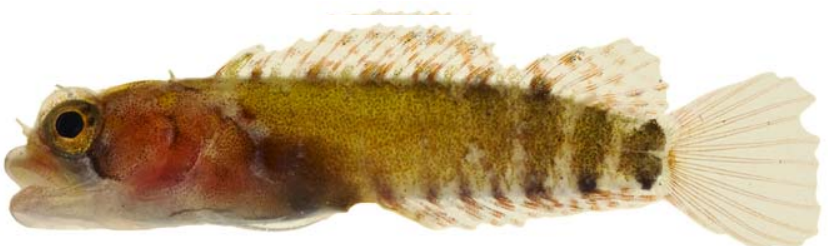

Figure 153. Starksia nanodes, $10.6 \mathrm{~mm} \mathrm{SL}$, adult male, photo by JT Williams.

doi:10.1371/journal.pone.0010676.g153

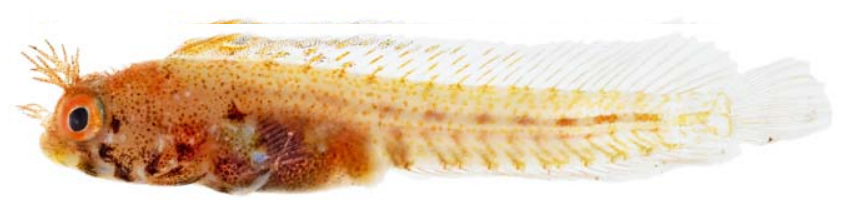

Figure 154. Acanthemblemaria aspera, $14.0 \mathrm{~mm} \mathrm{SL}$, juvenile/ female, photo by JT Williams.

doi:10.1371/journal.pone.0010676.g154

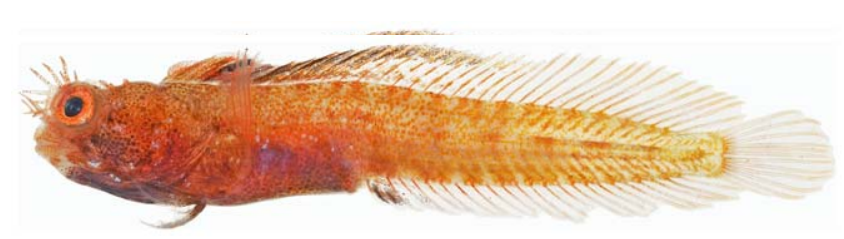

Figure 155. Acanthemblemaria aspera, $19.7 \mathrm{~mm} \mathrm{SL}$, adult male, photo by JT Williams.

doi:10.1371/journal.pone.0010676.g155 


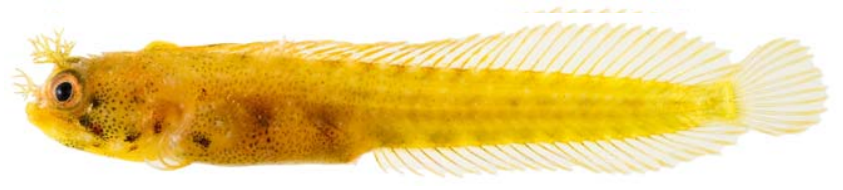

Figure 156. Acanthemblemaria aspera, $20.4 \mathrm{~mm} \mathrm{SL}$, yellow color morph (female?), photo by JT Williams.

doi:10.1371/journal.pone.0010676.g156

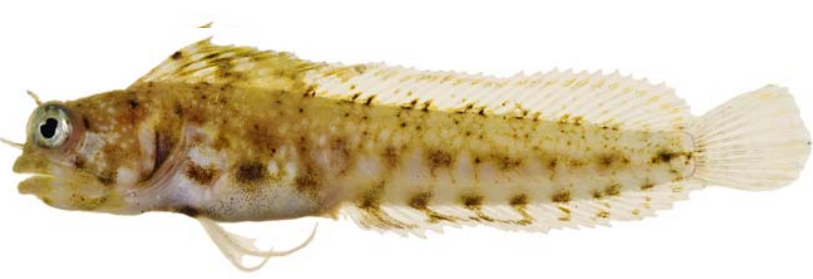

Figure 157. Emblemaria pandionis, $37.0 \mathrm{~mm} \mathrm{SL}$, adult female, photo by JT Williams.

doi:10.1371/journal.pone.0010676.g157

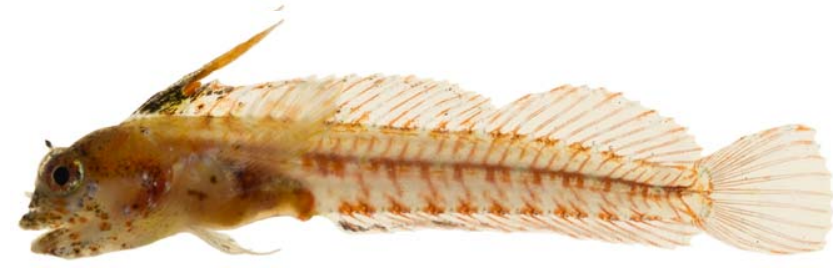

Figure 158. Emblemariopsis cf signifer, $16.2 \mathrm{~mm} \mathrm{SL}$, adult male, this specimen represents an undescribed species in the signifer species complex, photo by JT Williams.

doi:10.1371/journal.pone.0010676.g158

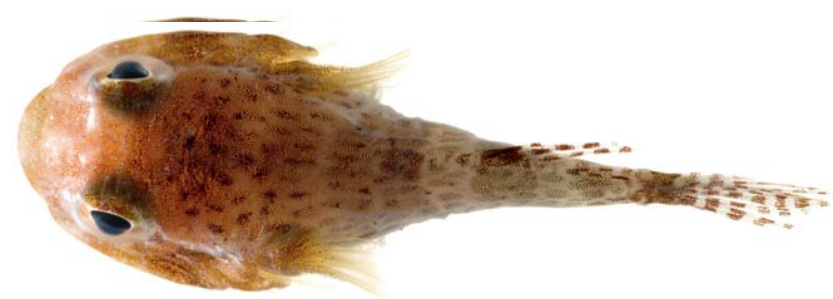

Figure 159. Acyrtus artius, 20.4 mm SL, dorsal view, photo by JT Williams.

doi:10.1371/journal.pone.0010676.g159

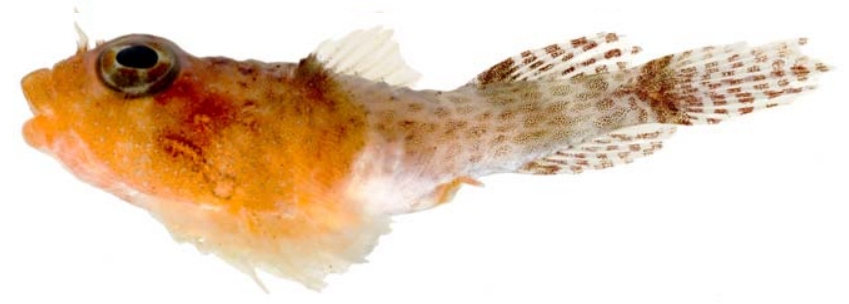

Figure 160. Acyrtus artius, 20.4 mm SL, lateral view, photo by JT Williams.

doi:10.1371/journal.pone.0010676.g160

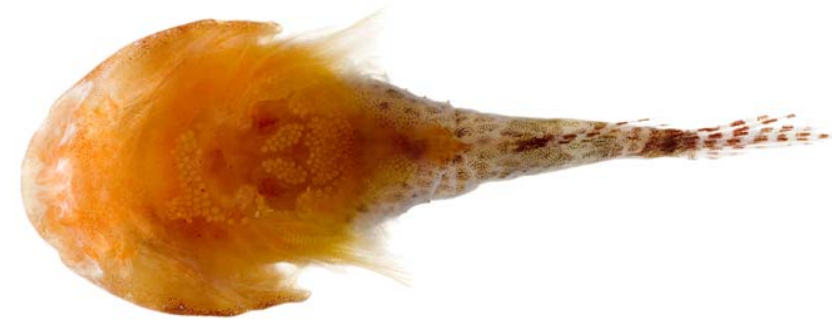

Figure 161. Acyrtus artius, $20.4 \mathrm{~mm} \mathrm{SL}$, ventral view showing pelvic disk, photo by JT Williams.

doi:10.1371/journal.pone.0010676.g161

Callionymidae-dragonets

Paradiplogrammus bairdi Jordan, 1888- lancer dragonet; USNM,

I, O; Figures 162, 163

Gobiidae-gobies

Coryphopterus dicrus Böhlke \& Robins, 1960 - colon goby; USNM, I; Figure 164

Coryphopterus eidolon Böhlke \& Robins, 1960-pallid goby;

USNM, I; Figure 165

Coryphopterus glaucofraenum Gill, 1863-bridled goby; USNM, I, V; Figure 166

Coryphopterus personatus (Jordan \& Thompson, 1905)-masked goby; USNM, I; Figure 167

Coryphopterus thrix Böhlke \& Robins, 1960-bartail goby; USNM, I; Figure 168

Elacatinus chancei (Beebe \& Hollister 1933)-shortstripe goby; USNM, I; Figure 169

Elacatinus evelynae (Böhlke \& Robins, 1968)—sharknose goby;

USNM, I; Figure 170

Elacatinus genie (Böhlke \& Robins, 1968)—cleaner goby; USNM, I

Evermannichthys metzelaari Hubbs, 1923 -roughtail goby; USNM, I; Figure 171

Gnatholepis thompsoni Jordan, 1904-goldspot goby; USNM, I, V; Figure 172

Lythrypnus elasson Böhlke \& Robins, 1960 - dwarf goby; USNM,

I; Figure 173

Lythrypnus minimus Garzón \& Acero P., 1988 - pygmy goby;

USNM, I; Figure 174

Lythrypnus nesiotes Böhlke \& Robins, 1960 — island goby; USNM,

I; Figure 175

Priolepis hipoliti (Metzelaar, 1922) - rusty goby; USNM, I; Figures 176, 177

Psilotris batrachodes Böhlke, 1963 -toadfish goby; USNM, I; Figure 178

Psilotris boehlkei Greenfield, 1993 -yellowspot goby; I; Figure 179

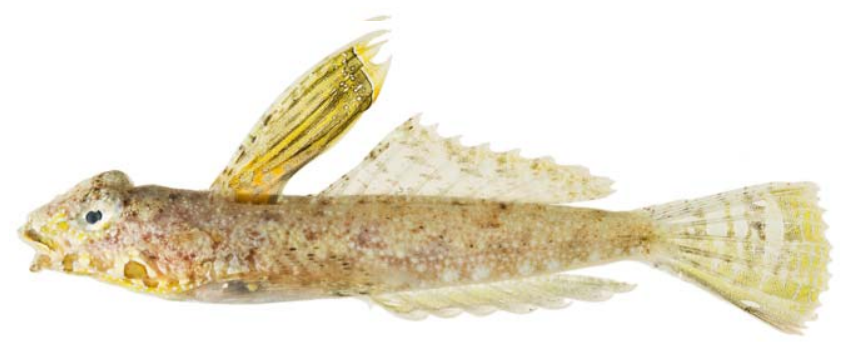

Figure 162. Paradiplogrammus bairdi, $39.8 \mathrm{~mm} \mathrm{SL}$, lateral view of an adult male, photo by JT Williams.

doi:10.1371/journal.pone.0010676.g162 


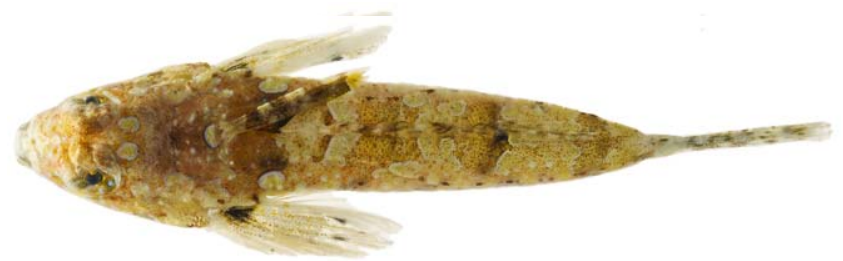

Figure 163. Paradiplogrammus bairdi, $29.8 \mathrm{~mm} \mathrm{SL}$, dorsal view of a smaller adult male, photo by JT Williams.

doi:10.1371/journal.pone.0010676.g163

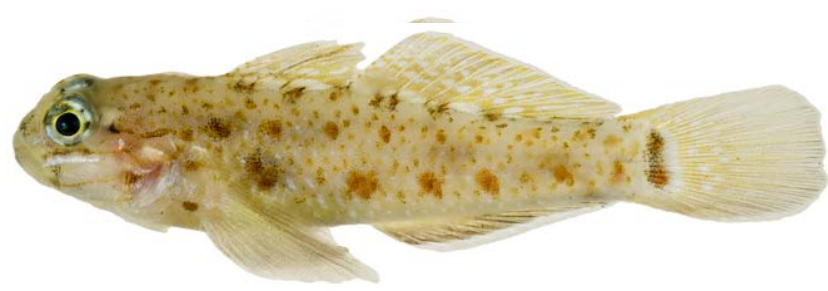

Figure 164. Coryphopterus dicrus, $31.8 \mathrm{~mm} \mathrm{SL}$, photo by JT Williams.

doi:10.1371/journal.pone.0010676.g164

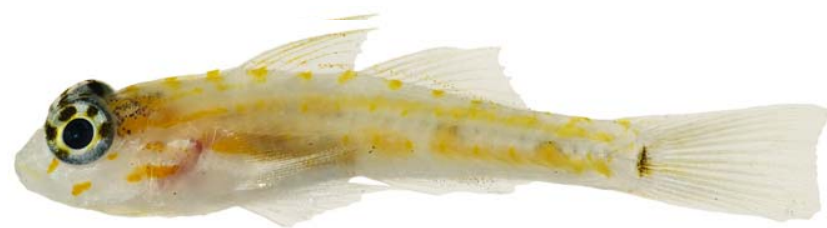

Figure 165. Coryphopterus eidolon, $18.3 \mathrm{~mm} \mathrm{SL,} \mathrm{photo} \mathrm{by} \mathrm{JT}$ Williams.

doi:10.1371/journal.pone.0010676.g165

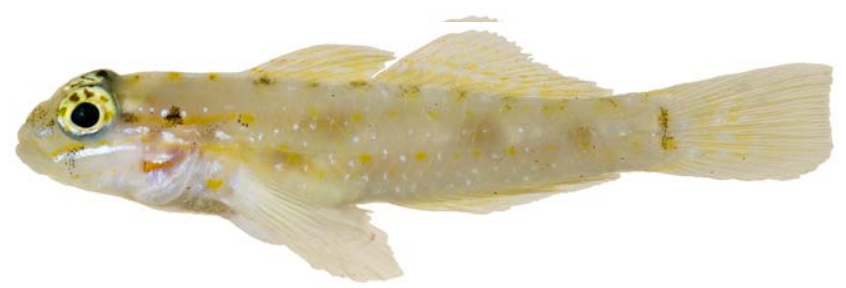

Figure 166. Coryphopterus glaucofraenum, $33.7 \mathrm{~mm} \mathrm{SL}$, photo by JT Williams.

doi:10.1371/journal.pone.0010676.g166

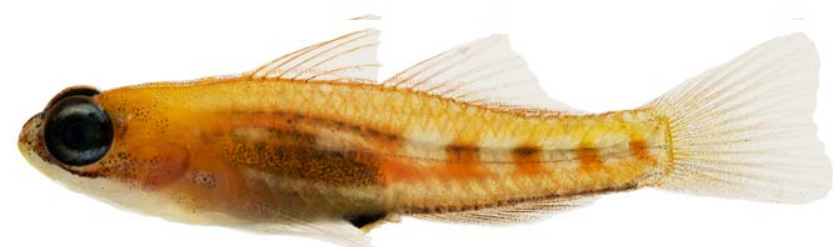

Figure 167. Coryphopterus personatus, $19.0 \mathrm{~mm} \mathrm{SL}$, photo by JT Williams.

doi:10.1371/journal.pone.0010676.g167

PLOS ONE I www.plosone.org
Figure 168. Coryphopterus thrix, $26.8 \mathrm{~mm} \mathrm{SL}$, photo by JT Williams.

doi:10.1371/journal.pone.0010676.g168

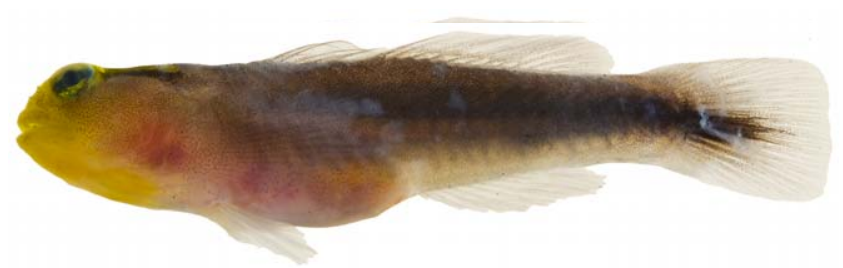

Figure 169. Elacatinus chancei, $28.1 \mathrm{~mm} \mathrm{SL}$, photo by JT Williams.

doi:10.1371/journal.pone.0010676.g169

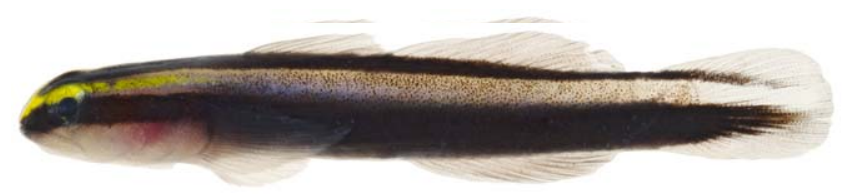

Figure 170. Elacatinus evelynae, $27.7 \mathrm{~mm} \mathrm{SL}$, photo by JT Williams.

doi:10.1371/journal.pone.0010676.g170

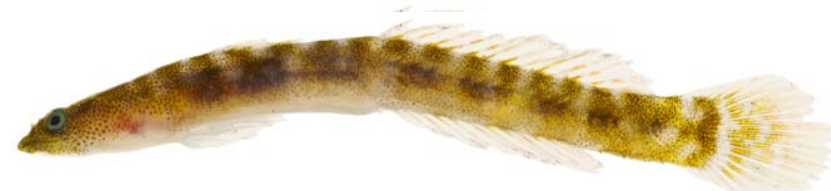

Figure 171. Evermannichthys metzelaari, $21.0 \mathrm{~mm} \mathrm{SL}$, photo by JT Williams.

doi:10.1371/journal.pone.0010676.g171

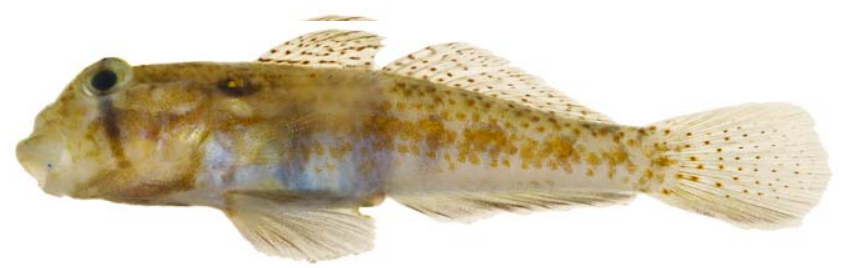

Figure 172. Gnatholepis thompsoni, $31.5 \mathrm{~mm} \mathrm{SL}$, photo by JT Williams.

doi:10.1371/journal.pone.0010676.g172 


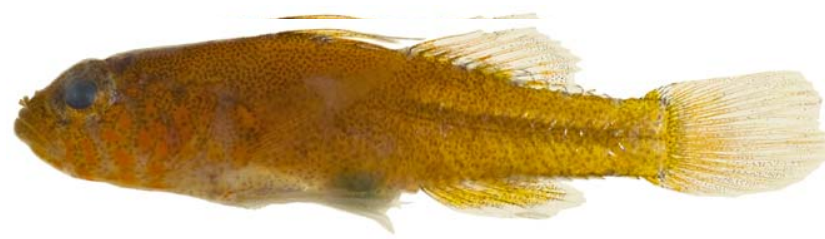

Figure 173. Lythrypnus elasson, $9.2 \mathrm{~mm} \mathrm{SL}$, adult male, photo by JT Williams.

doi:10.1371/journal.pone.0010676.g173

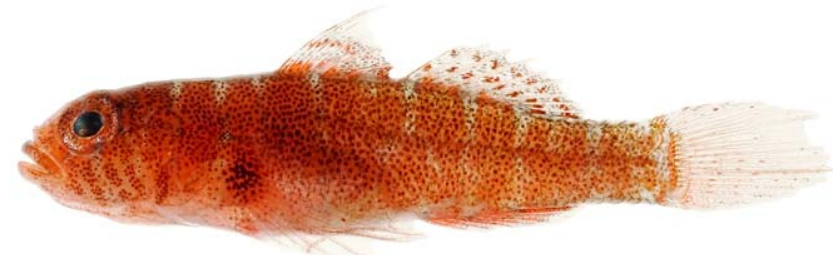

Figure 174. Lythrypnus minimus, $10.6 \mathrm{~mm} \mathrm{SL}$, adult male, photo by JT Williams.

doi:10.1371/journal.pone.0010676.g174

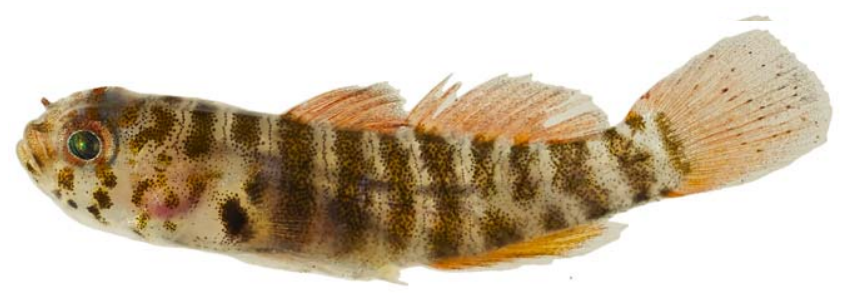

Figure 175. Lythrypnus nesiotes, $11.9 \mathrm{~mm} \mathrm{SL}$, adult male, photo by JT Williams.

doi:10.1371/journal.pone.0010676.g175

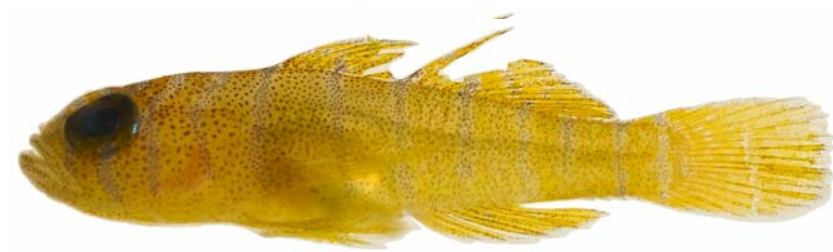

Figure 176. Priolepis hipoliti, $12.2 \mathrm{~mm} \mathrm{SL}$, female, photo by JT Williams.

doi:10.1371/journal.pone.0010676.g176

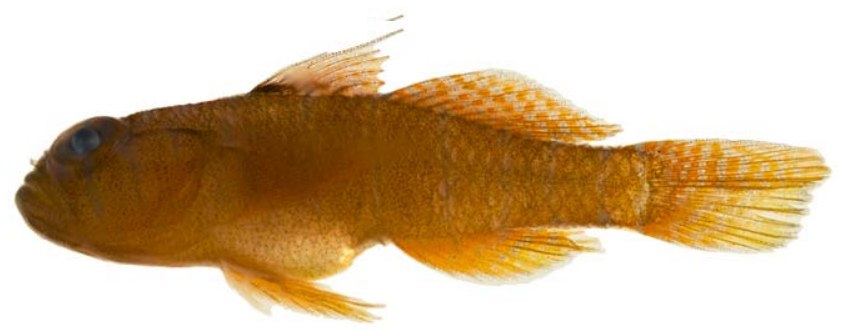

Figure 177. Priolepis hipoliti, $18.7 \mathrm{~mm} \mathrm{SL}$, adult male, photo by JT Williams.

doi:10.1371/journal.pone.0010676.g177

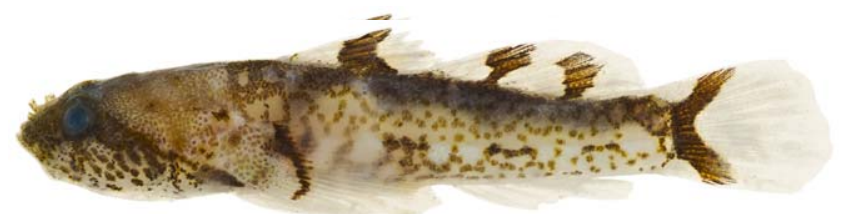

Figure 178. Psilotris batrachodes, $13.3 \mathrm{~mm} \mathrm{SL}$, adult, photo by JT Williams.

doi:10.1371/journal.pone.0010676.g178

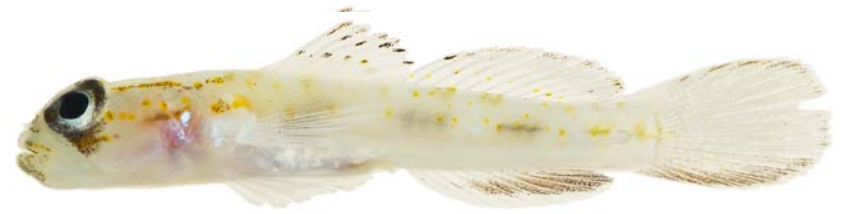

Figure 179. Psilotris boehlkei, $26.5 \mathrm{~mm} \mathrm{SL}$, adult, photo by JT Williams.

doi:10.1371/journal.pone.0010676.g179

The fresh colors of P. boehlkei are presented (Figure 179) for the first time. This very rare species was previously known from only five specimens taken at St. Barthelemey in 1965. The single specimen we collected extends the known distribution of $P$. boehlkei to Saba Bank. We have named this species the yellowspot goby in reference to the yellow spots on the head and body.

Pycnomma roosevelti Ginsburg, 1939 - Roosevelt's goby; USNM, I; Figure 180

Although $P$. roosevelti has previously been taken from a several scattered localities around the Caribbean (Isla Providencia, Guadeloupe, Belize and Puerto Rico), there are fewer than 10 specimens known and its fresh colors (Figure 180) have not been published previously.

Risor ruber (Rosén, 1911) — tusked goby; USNM, I; Figure 181

Acanthuridae-surgeonfishes

Acanthurus bahianus Castelnau, 1855 - ocean surgeon; USNM, I, O, V; Figure 182

Acanthurus chirurgus (Bloch, 1787)-doctorfish; USNM, I, O, V; Figure 183

Acanthurus coeruleus Bloch \& Schneider, 1801-blue tang; USNM, I, O, V; Figure 184

Sphyraenidae-barracudas

Sphyraena barracuda (Walbaum, 1792)—great barracuda; $\mathbf{O}, \mathbf{V}$

Scombridae-mackerels

Acanthocybium solandri (Cuvier, 1832) —wahoo; F

Euthynnus alletteratus (Rafinesque, 1810) - little tunny; USNM; Figure 185

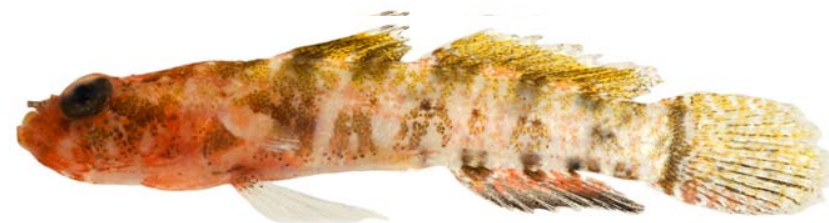

Figure 180. Pycnomma roosevelti, $14.6 \mathrm{~mm} \mathrm{SL}$, adult, photo by JT Williams.

doi:10.1371/journal.pone.0010676.g180 


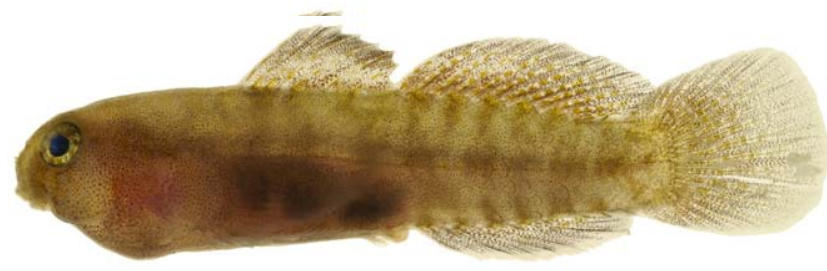

Figure 181. Risor ruber, $18.7 \mathrm{~mm} \mathrm{SL}$, adult, photo by JT Williams.

doi:10.1371/journal.pone.0010676.g181

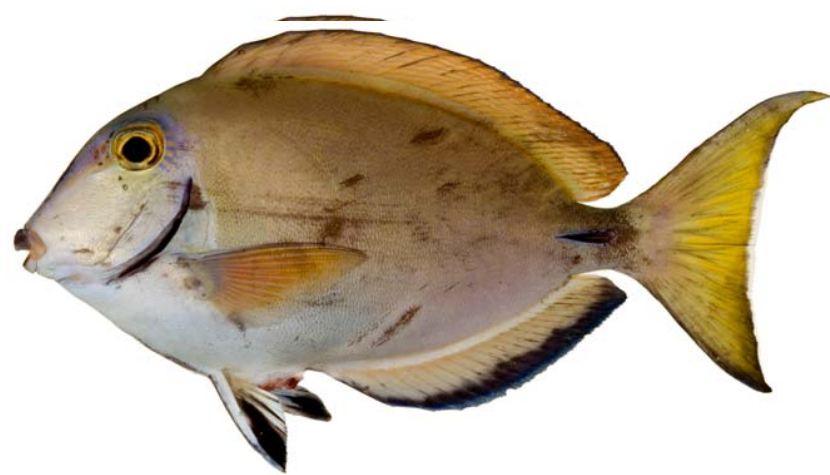

Figure 182. Acanthurus bahianus, $128.2 \mathrm{~mm} \mathrm{SL}$, photo by JT Williams.

doi:10.1371/journal.pone.0010676.g182

Scomberomorus regalis (Bloch, 1793) - cero; USNM

Thunnus atlanticus (Lesson 1831)_blackfin tuna; F; Figure 186

\section{Caproidae-boarfishes}

Antigonia capros Lowe, 1843-deepbody boarfish; USNM, F; Figure 187

Antigonia combatia Berry \& Rathjen 1959 - shortspine boarfish; UF, T

The shortspine boarfish record from Saba Bank is based on a trawl collection with vouchered museum specimens at UF.

Bothidae-lefteye flounders

Bothus lunatus (Linnaeus, 1758) — peacock flounder; $\mathbf{O}$

Bothus ocellatus (Agassiz, 1831) - eyed flounder; UF, USNM, I; Figure 188

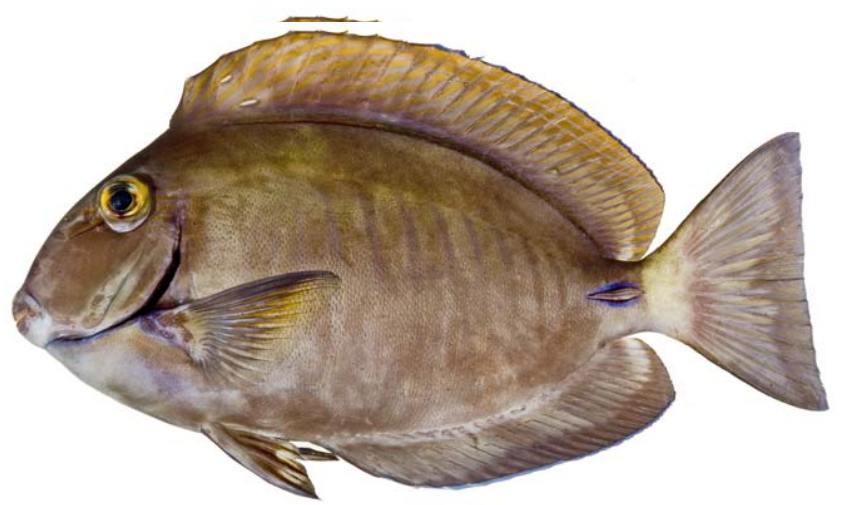

Figure 183. Acanthurus chirurgus, $168.0 \mathrm{~mm} \mathrm{SL}$, photo by JT Williams.

doi:10.1371/journal.pone.0010676.g183

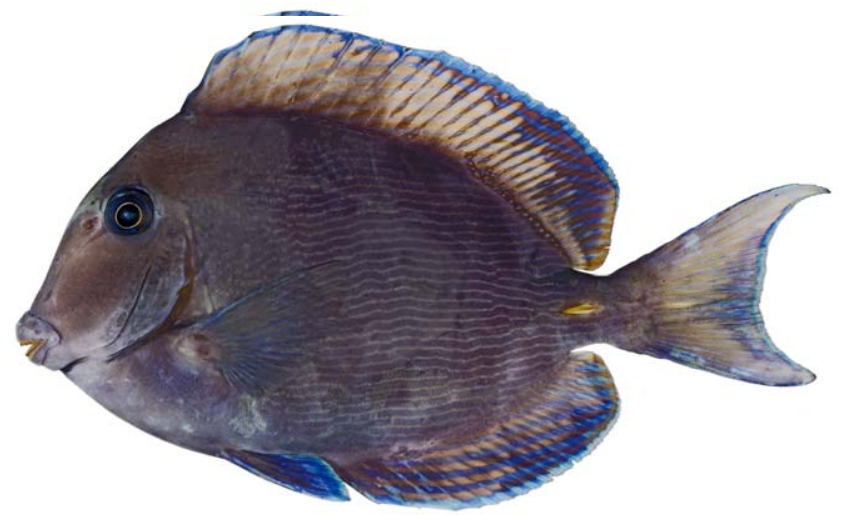

Figure 184. Acanthurus coeruleus, $146.2 \mathrm{~mm} \mathrm{SL}$, photo by JT Williams.

doi:10.1371/journal.pone.0010676.g184

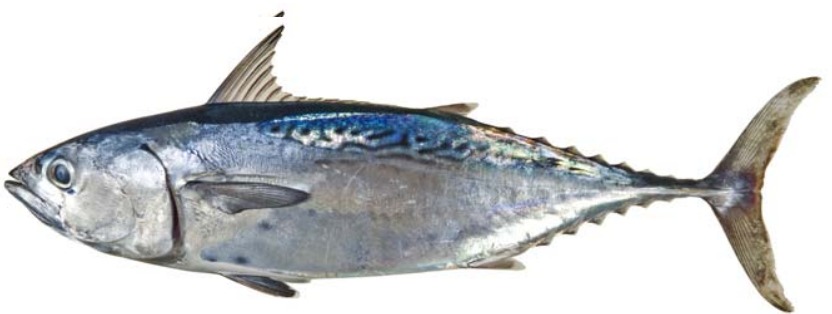

Figure 185. Euthynnus alletteratus, $355.1 \mathrm{~mm} \mathrm{FL,} \mathrm{photo} \mathrm{by} \mathrm{JT}$ Williams.

doi:10.1371/journal.pone.0010676.g185

Trichopsetta ventralis (Goode \& Bean, 1885)—sash flounder; UF, $\mathbf{T}$

Paralichthyidae-sand flounders

Citharicthys dinoceros Goode \& Bean, 1886 — spined whiff; USNM, T

Cynoglossidae-tonguefishes

Symphurus arawak Robins \& Randall, 1965-Caribbean tonguefish; USNM, I; Figure 189

Symphurus ommaspilus Böhlke, 1961-ocellated tonguefish; USNM, I; Figure 190

\section{Balistidae-triggerfishes}

Balistes vetula Linnaeus, 1758 - queen triggerfish; USNM, O, V Canthidermis sufflamen (Mitchill, 1815) - ocean triggerfish; $\mathbf{O}, \mathbf{V}$ Melichthys niger (Bloch, 1786) - black durgon; USNM, O, V

Xanthichthys ringens (Linnaeus, 1758) — sargassum triggerfish; USNM, I; Figure 191

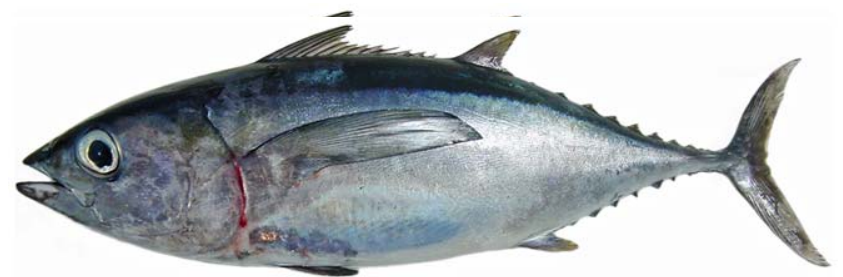

Figure 186. Thunnus atlanticus, $516 \mathbf{~ m m ~ F L , ~ p h o t o ~ b y ~ W ~ T o l l e r . ~}$ doi:10.1371/journal.pone.0010676.g186 


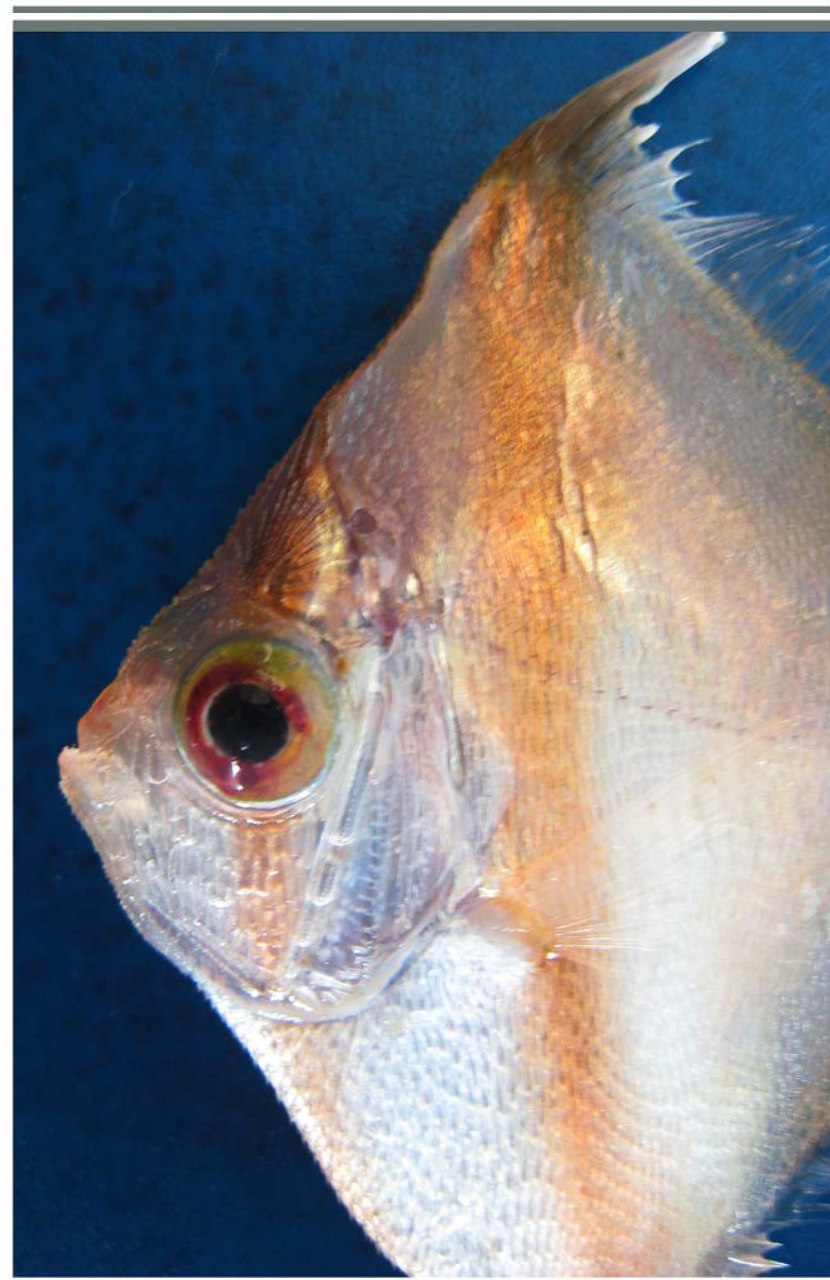

Figure 187. Antigonia capros, $37 \mathrm{~mm} \mathrm{SL}$, close-up of head, photo by $\mathbf{W}$ Toller.

doi:10.1371/journal.pone.0010676.g187

\section{Monacanthidae-filefishes}

Aluterus scriptus (Osbeck, 1765) - scrawled filefish; USNM, F, V Cantherhines macrocerus (Hollard, 1853) — whitespotted filefish; USNM, F; Figure 192

Cantherhines pullus (Ranzani, 1842) - orangespotted filefish; $\mathbf{O}, \mathbf{V}$

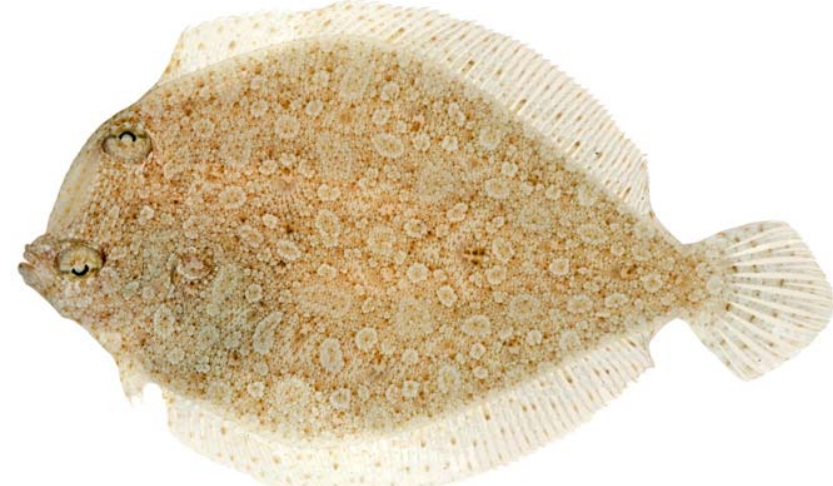

Figure 188. Bothus ocellatus, $95.8 \mathrm{~mm} \mathrm{SL}$, photo by JT Williams. doi:10.1371/journal.pone.0010676.g188

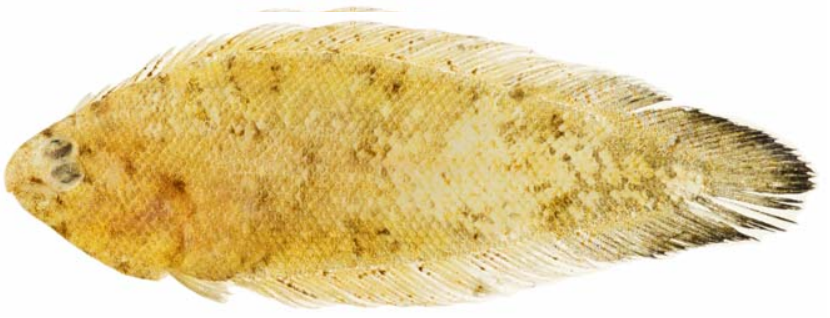

Figure 189. Symphurus arawak, $30.7 \mathrm{~mm} \mathrm{SL}$, photo by JT Williams.

doi:10.1371/journal.pone.0010676.g189

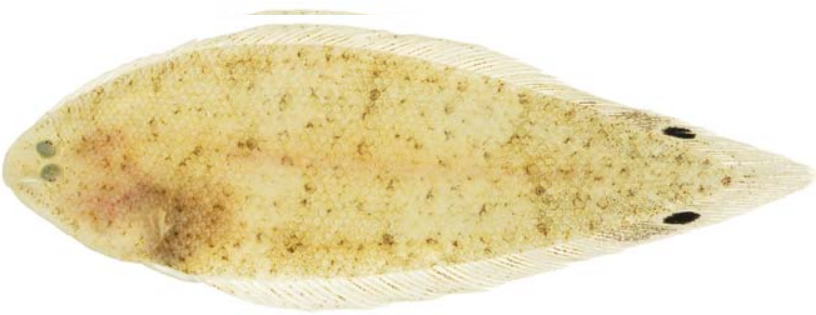

Figure 190. Symphurus ommaspilus, $42.9 \mathrm{~mm} \mathrm{SL}$, photo by JT Williams.

doi:10.1371/journal.pone.0010676.g190

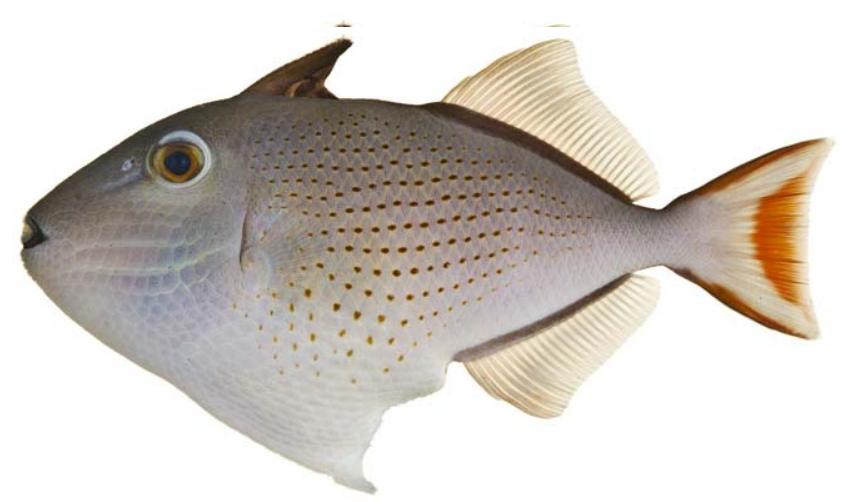

Figure 191. Xanthichthys ringens, $87.3 \mathrm{~mm} \mathrm{SL}$, photo by JT Williams.

doi:10.1371/journal.pone.0010676.g191

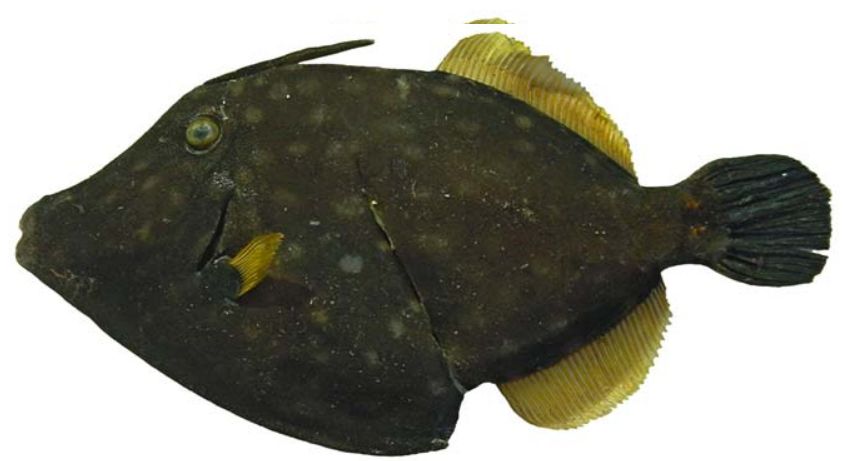

Figure 192. Cantherhines macrocerus, $273 \mathrm{~mm} \mathrm{SL}$, image flipped horizontally - right side is shown, photo by $\mathbf{W}$ Toller. doi:10.1371/journal.pone.0010676.g192 
Monacanthus ciliatus (Mitchill, 1818) — fringed filefish; USNM, I, V; Figure 193

Monacanthus tuckeri Bean, 1906-slender filefish; USNM, I; Figure 194

\section{Ostraciidae-boxfishes}

Acanthostracion polygonia Poey, 1876-honeycomb cowfish;

USNM, F, O, V

Acanthostracion quadricornis (Linnaeus, 1758)—scrawled cowfish; USNM, F, $\mathbf{O}$

Lactophrys bicaudalis (Linnaeus, 1758) — spotted trunkfish; F

Lactophrys trigonus (Linnaeus, 1758) - trunkfish; USNM, I, V; Figure 195

Lactophrys triqueter (Linnaeus, 1758) - smooth trunkfish; USNM,

F, OBS, VIS; Figure 196

Tetraodontidae-puffers

Canthigaster rostrata (Bloch, 1786) - sharpnose puffer; USNM, I, O, V; Figure 197

Sphoeroides spengleri (Bloch, 1785) — bandtail puffer; USNM, I, O; Figure 198

\section{Diodontidae-porcupinefishes}

Chilomycterus antillarum Jordan \& Rutter, 1897-web burrfish; USNM, F; Figure 199

Diodon holocanthus Linnaeus, 1758-balloonfish; USNM, I, V; Figure 200

Diodon hystrix Linnaeus, 1758-porcupinefish; $\mathbf{O}$

\section{Discussion}

We document the occurrence of 270 species of fishes at Saba Bank. The diversity of fishes at Saba Bank is comparable (Table 1) to that of the oceanic atolls of Colombia (273 species), the islands in the Mona Passage of Puerto Rico (261 species) and Buck Island Reef National Monument (BIRNM; 262 species). The relatively high diversity of fishes at Saba Bank exists despite the lack of emergent land at the bank. There is no shallow-water shore-fish fauna represented on the bank due to the absence of a high-energy shoreline. These habitats typically add significantly to the fish diversity of Caribbean habitats. For example, the tube blennies (family Chaenopsidae) are a group of shorefishes typically found in fairly shallow coastal waters. According to Williams [4], there are approximately 22 recognized species of tube blennies known to

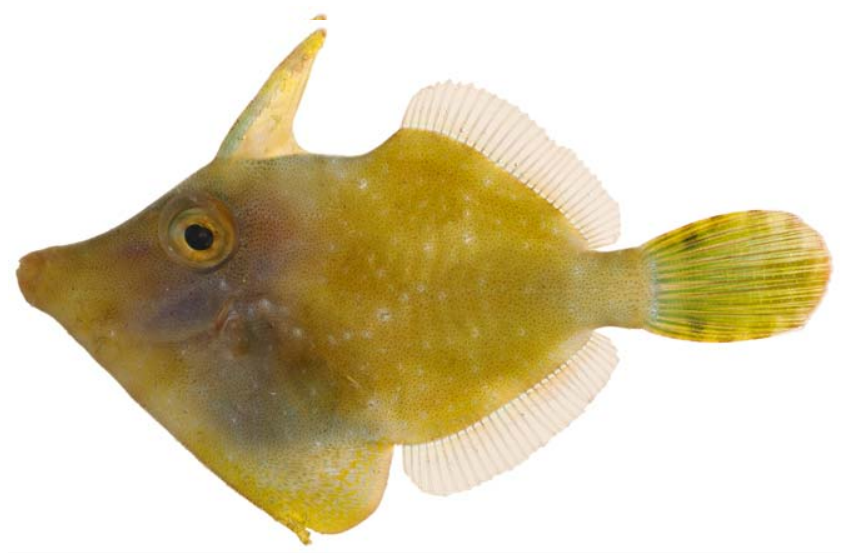

Figure 193. Monacanthus ciliatus, $33.4 \mathrm{~mm} \mathrm{SL}$, photo by JT Williams.

doi:10.1371/journal.pone.0010676.g193

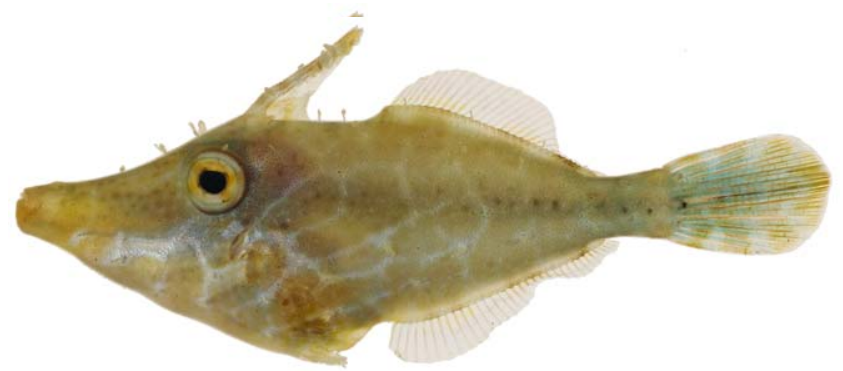

Figure 194. Monacanthus tuckeri, $32.3 \mathrm{~mm} \mathrm{SL}$, photo by JT Williams.

doi:10.1371/journal.pone.0010676.g194

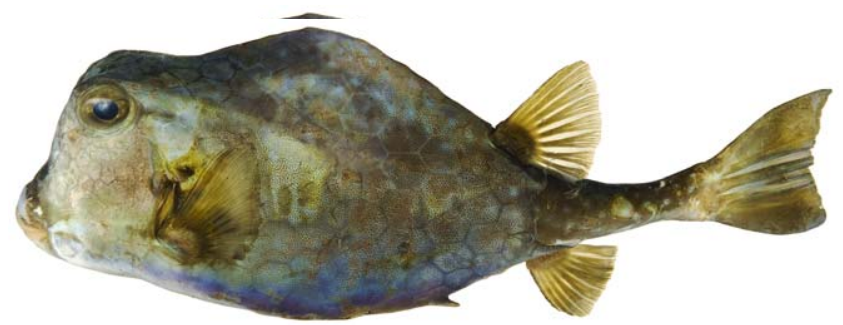

Figure 195. Lactophrys trigonus, $293.1 \mathrm{~mm} \mathrm{SL}$, photo by JT Williams.

doi:10.1371/journal.pone.0010676.g195

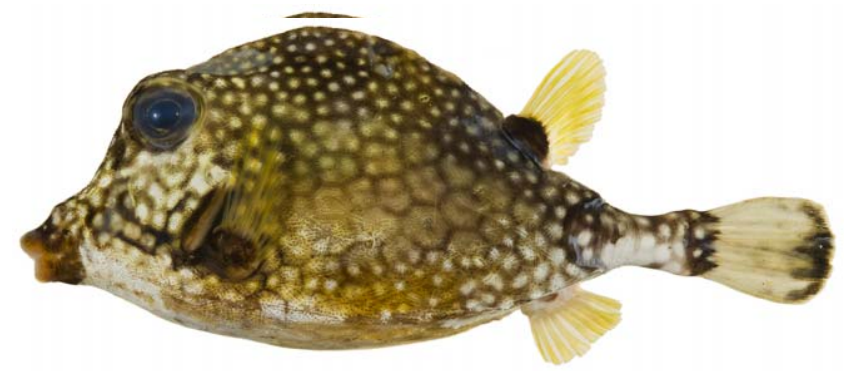

Figure 196. Lactophrys triqueter, $111.7 \mathrm{~mm} \mathrm{SL}$, photo by JT Williams.

doi:10.1371/journal.pone.0010676.g196

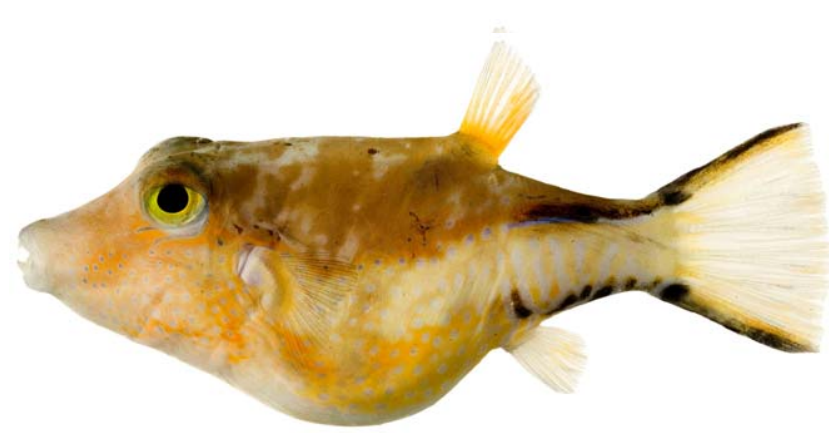

Figure 197. Canthigaster rostrata, $50.9 \mathrm{~mm} \mathrm{SL}$, photo by JT Williams.

doi:10.1371/journal.pone.0010676.g197 


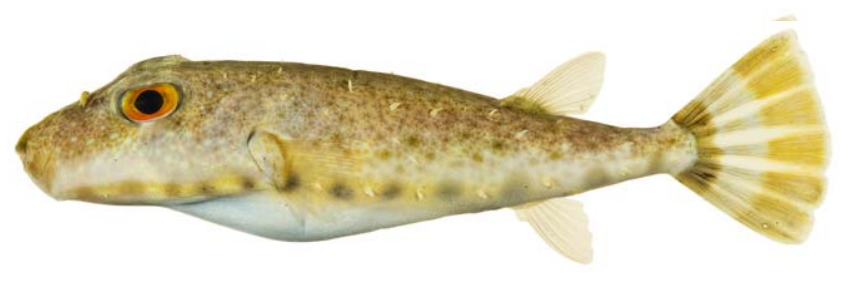

Figure 198. Sphoeroides spengleri, $73.1 \mathrm{~mm} \mathrm{SL}$, photo by JT Williams.

doi:10.1371/journal.pone.0010676.g198

occur in the central Caribbean, but only three of these species were found on Saba Bank. In addition, the absence of mangrove vegetation and apparent lack of sea-grass beds also limits the fish fauna of Saba Bank. In their quantitative study of a number of Saba Bank habitats, Toller et al. [5] attribute the apparent lack or rarity of a number of fish species found on Saba Bank to the absence of those habitats as nurseries for the juvenile stages of these fish species. Nevertheless, diverse habitat types exist on Saba Bank ranging from coral reefs and algal flats to soft-bottom lagoon areas and scoured hard, flat, pavement-like zones. These diverse habitats support a highly diverse, fish fauna and include a number of undescribed, new species along with species rarely encountered elsewhere in the Caribbean.

The highly diverse, fish fauna so far reported appears to substantially under represent the species richness of fishes for Saba Bank. The actual species-accumulation curve of rotenone collections and visual surveys combined do not reach an asymptote (Figure 201). The expected species-accumulation curves (Chao2 and Jackl estimators in EstimateS) predict total fish-species richness somewhere between 320 and 411 species.

Of the 270 species we report (Table 1) from Saba Bank, 132 $(49 \%)$ were observed during visual surveys. This result is comparable to the findings for Navassa Island where $41 \%$ of the fish species were detected by visual surveys [6], for the Mona Passage islands with $43 \%$ of the fish species detected visually [7], and for BIRNM with $44 \%$ of the fish species detected visually [8]. A higher percentage of fish species was detected visually at Saba Bank than at Navassa, Mona, or BIRNM. This could possibly be a result of the higher number of visual surveys carried out at Saba Bank than at Navassa or Mona. Although the BIRNM results are based on a higher number of visual surveys (70), a comparable percentage of the fauna was detected visually at BIRNM. BIRNM ichthyocide surveys (58) appear to demonstrate the ability of ichthyocide collections to more thoroughly sample the fauna (see below). Visual censuses have numerous biases as discussed in the methods for the Pelican Cays study [9]. Despite the slight differences in methods and sampling designs between the published fish species-richness studies in different parts of the

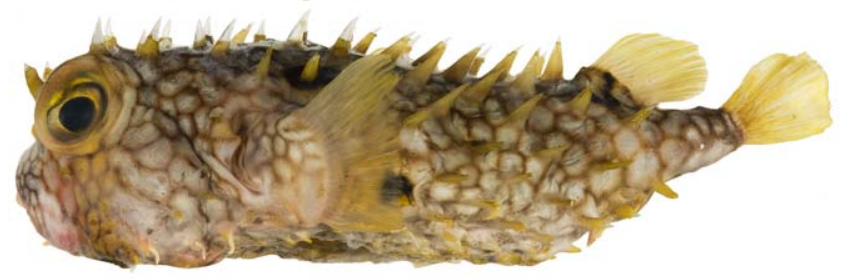

Figure 199. Chilomycterus antillarum, $180.0 \mathrm{~mm} \mathrm{SL}$, photo by JT Williams.

doi:10.1371/journal.pone.0010676.g199

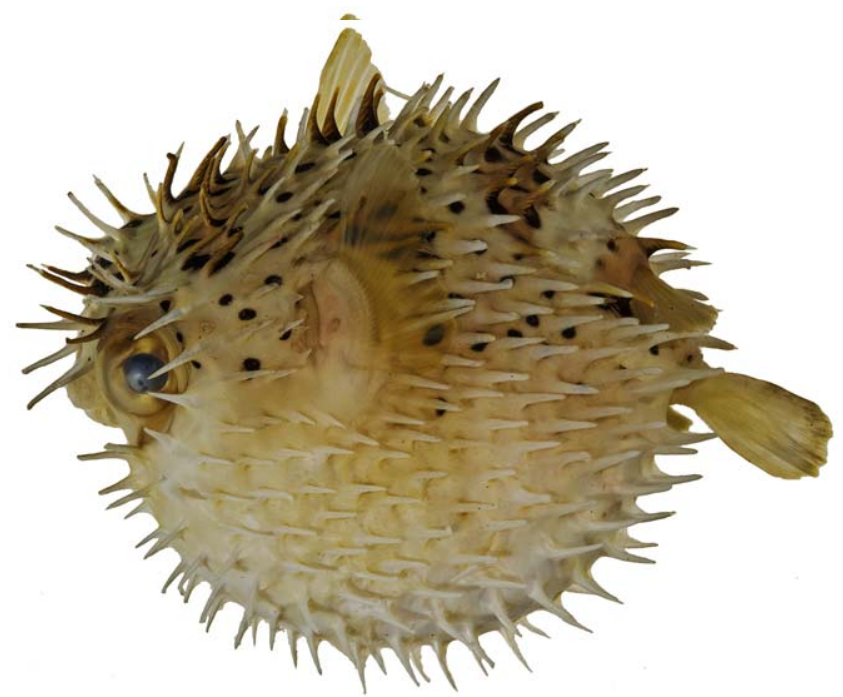

Figure 200. Diodon holocanthus, $104.9 \mathrm{~mm} \mathrm{SL}$, photo by JT Williams.

doi:10.1371/journal.pone.0010676.g200

Caribbean, the methods utilized produce consistent and comparable results.

Ichthyocide collections at Saba Bank yielded specimens representing 155 fish species (57\% of the total fish fauna). At Navassa Island where there were fewer visual surveys than at Saba Bank, over $70 \%$ of the fish species were collected with ichthyocide [6], at the Mona Passage islands, 61\% of the fish species were collected with ichthyocide [7], and at BIRNM, 87\% of the fish species were collected with ichthyocide despite their being more visual surveys in the BIRNM study [8]. By occupying 58 (27 at Saba Bank) ichthyocide stations covering a broader diversity of ecological habitats, the BIRNM study appears to have obtained a more thorough sampling of the resident species. The lower number of species taken at Saba Bank with ichthyocide is at least partially due to the absence of shallow habitat. Shallow habitat is required by many of the small cryptic fish species that are normally detected only with the use of ichthyocide and these species have not been found at Saba Bank. The prevalence of strong currents across Saba Bank further limits the effectiveness of ichthyocide, which is only effective when relatively high concentrations of ichthyocide remain in one place (preferably in a confined area) for more than 15 to 20 minutes. The ichthyocide-collecting methods employed at Saba Bank were the same as those used at Navassa Island and Belize. The BIRNM ichthyocide collecting methods differed from ours only in that the BIRNM study used a block net in addition to collecting outside the net. Nevertheless, the BIRNM methods were comparable to ours because the BIRNM study included those species collected outside the block nets in their results. There are inherent biases in ichthyocide collecting due to unpredictability of environmental parameters, such as currents, temperature, degree of confinement of the sampling area, ability of larger species to swim away, and variable assays of active rotenone in the powdered Derris root (batches often vary from $5 \%$ to over $11 \%$ active rotenone due to natural variation in rotenone concentration among the roots of different plants). Despite these biases, ichthyocide collections yield comparable results as described above.

Neither visual surveying nor ichthyocide collecting alone are capable of providing a comprehensive survey of coastal (or submerged atoll in the case of Saba Bank) fish species. A 
Table 1. Number of fishes recorded at well sampled sites in the Greater Caribbean.

\begin{tabular}{lll}
\hline & & \\
\hline Site & No. fish species & Source \\
\hline Alligator Reef, FL & 517 & Starck [12] \\
Dry Tortugas, FL & 442 & Longley \& Hildebrand [13] \\
Bermuda & 433 & Smith-Vaniz et al [14] \\
St. Croix & 400 & Clavijo et al [15] \\
Barrier Reef, Belize & 339 & C.L. Smith et al [9] \\
Offshore Banks, Belize & 293 & C.L. Smith et al [9] \\
Oceanic Atolls, Colombia & 273 & Mejia et al [16] \\
Saba Bank & 270 & This paper \\
Buck Island Reef & 262 & Smith-Vaniz et al [8] \\
Mona Passage Islands, Puerto Rico & 261 & Dennis et al [7] \\
Flower Garden Bank Nat. Mar. Sanctuary, Texas, USA & 240 & E. Hickerson, NOAA, pers. comm., Dec. 2009 \\
Navassa Island, USA & 237 & Collette et al [6] \\
Rhomboidal Cays, Belize & 193 & C.L. Smith et al [9] \\
Core Pelican Cays, Belize & 168 & C.L. Smith et al [9] \\
Peripheral Rhomboidal Cays, Belize & 123 & C.L. Smith et al [9] \\
\hline doi:10.1371/journal.pone.0010676.t001 & &
\end{tabular}

combination of visual surveys, ichthyocide sampling using SCUBA, and various fishing techniques must be employed to effectively assess fish-species richness in marine habitats shallow enough to be accessible to SCUBA divers.

The number of fish species living on Saba Bank is undoubtedly higher than 270 as indicated by Chao 2 and Jackl estimators. As most parts of Saba Bank are deeper than $25 \mathrm{~m}$, sampling with ichthyocides using SCUBA is limited by the reduced bottom time at these depths. As a result we focused primarily on the rim

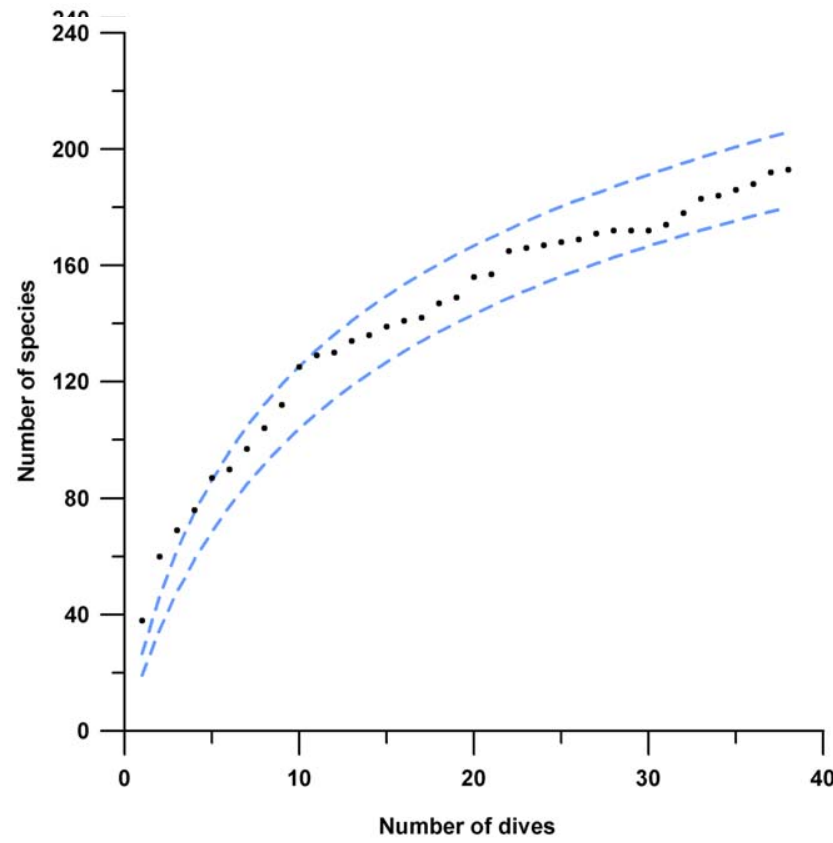

Figure 201. Actual species-accrual curve (black dots) for 38 dives collecting and identifying fish species on Saba Bank. Sobs (Mao Tau) 95\% confidence intervals [8] are shown as light blue dashed lines.

doi:10.1371/journal.pone.0010676.g201 (shallowest parts) of the submerged atoll to maximize bottom time for collecting. Future sampling with ichthyocides applied by divers utilizing rebreathers, supplemented with trawl and dredge sampling would allow collecting from the outer slopes and would certainly yield additional new and interesting species of fishes from this submerged atoll.

\section{Methods}

The NMNH/SI Animal Care and Use Committee approved the methods and procedures utilized during the course of this biodiversity assessment project. All Saba Bank projects had collecting permits through the Convention on Trade in Endangered Species (CITES, where necessary) and the Saba Conservation Foundation (where CITES was not required).

Roving surveys were completed using SCUBA and lasted 60 minutes, bottom time permitting. All species encountered were listed on a slate while swimming in a haphazard pattern covering all bottom depths possible down to a maximum of $38 \mathrm{~m}$. Other visual surveys are described in Toller et al [5]. Collecting methods follow Collette et al [6]. Species were photographed in aquaria after the fins were pinned out and brushed with formaldehyde solution. Tissue samples were taken from fresh specimens and the voucher specimens were preserved in a formaldehyde solution diluted with water to $3.75 \%$ formaldehyde. Large specimens were also injected with $37.5 \%$ formaldehyde before being soaked in the $3.75 \%$ formaldehyde solution.

After arrival at the Museum Support Center (MSG), National Museum of Natural History, specimens were transferred sequentially through water-diluted solutions of $25 \%$ ethanol, $50 \%$ ethanol, and finally into $75 \%$ ethanol for permanent archival storage. Specimens were then processed and cataloged into the USNM at the MSG in Suitland, MD.

To generate species-accumulation curves, a data matrix of presence/absence was constructed from 38 combined roving surveys, rotenone collections, and fish-habitat transects using species as variables and dives as observations (Table 2 provides details for each survey and collecting station). The matrix was employed for actual and expected species-accumulation curves 
Table 2. Collecting stations occupied on Saba Bank, 2006-2007.

\begin{tabular}{|c|c|c|c|c|c|c|}
\hline Station number & $\begin{array}{l}\text { Roving \& } \\
\text { Rotenone }\end{array}$ & Coordinates & Date & Depth (m) & Local name & Description of habitat \\
\hline Saba-06-01 & RR01 & $\begin{array}{l}17028.778 \mathrm{~N} \\
63013.663 \mathrm{~W}\end{array}$ & 04 Jan 2006 & 30 & North East Reef & $\begin{array}{l}\text { N.E. reefs, just S of Poison Bank. Spur } \\
\text { and groove reef, low relief, many } \\
\text { algae (Dictyota)-rotenoned a groove } \\
\text { in reef with sand at bottom, walls of } \\
\text { groove with low corals }\end{array}$ \\
\hline Saba-06-02 & RR02 & $\begin{array}{l}17026.883 \mathrm{~N} \\
63054.055 \mathrm{~W}\end{array}$ & 05 Jan 2006 & 38 & Small Bank South & $\begin{array}{l}\text { Small Bank South. Scattered corals, } \\
\text { many Xestospongia. Nassau grouper, } \\
\text { yellowfin grouper, and black grouper } \\
\text { sighted. Nurse shark-rotenoned area } \\
\text { with encrusting corals }\end{array}$ \\
\hline Saba-06-03 & RR03 & $\begin{array}{l}17 \mathrm{o} 25.778 \mathrm{~N} \\
63041.037 \mathrm{~W}\end{array}$ & 05 Jan 2006 & 35 & Rhodolith Reef & $\begin{array}{l}\text { Western patch reef, many rodoliths- } \\
\text { rotenoned a flat area with sponges } \\
\text { and rubble bottom }\end{array}$ \\
\hline Saba-06-04 & & $\begin{array}{l}17018.557 \mathrm{~N} \\
63022.019 \mathrm{~W}\end{array}$ & 05 Jan 2006 & 35 & & Fish Trap- Local Fishermen \\
\hline Saba-06-05 & RR04 & $\begin{array}{l}17 \mathrm{o} 24.602 \mathrm{~N} \\
63011.748 \mathrm{~W}\end{array}$ & 06 Jan 2006 & 27 & Redman Bulge & $\begin{array}{l}\text { Eastern reef, spur and groove, } \\
\text { medium height, many algae (dictyota) }\end{array}$ \\
\hline Saba-06-06 & RR05 & $\begin{array}{l}17026.028 \mathrm{~N} \\
63016.536 \mathrm{~W}\end{array}$ & 06 Jan 2006 & 22 & Seaweed city & $\begin{array}{l}\text { pavement covered with ca. } 10 \mathrm{~cm} \\
\text { sand layer, Pseudopterogorgia, many } \\
\text { algae species }\end{array}$ \\
\hline Saba-06-07 & & $\begin{array}{l}17033.697 \mathrm{~N} \\
63046.949 \mathrm{~W}\end{array}$ & & $0-1$ & & $\begin{array}{l}\text { Caught on hook and line while trolling } \\
\text { over Saba Bank }\end{array}$ \\
\hline Saba-06-08 & RR06 & $\begin{array}{l}17033.092 \mathrm{~N} \\
63028.758 \mathrm{~W}\end{array}$ & 07 Jan 2006 & 37 & Grouper Bank & $\begin{array}{l}\text { bare pavement, occasional solution } \\
\text { holes, Manicinias, many conch (old) }\end{array}$ \\
\hline Saba-06-09 & RR07 & $\begin{array}{l}17034.893 \mathrm{~N} \\
63024.400 \mathrm{~W}\end{array}$ & 07 Jan 2006 & 30 & Rendezvous Hill & $\begin{array}{l}\text { pavement with corals, low relief- } \\
\text { rotenoned a low-relief coral reef with } \\
\text { soft and hard corals, and sponges }\end{array}$ \\
\hline Saba-06-10 & & $\begin{array}{l}17025.883 \mathrm{~N} \\
63021.874 \mathrm{~W}\end{array}$ & 08 Jan 2006 & $0-1$ & & $\begin{array}{l}\text { Caught on hook and line while trolling } \\
\text { over Saba Bank }\end{array}$ \\
\hline Saba-06-11 & RR08 & $\begin{array}{l}17014.070 \mathrm{~N} \\
63026.915 \mathrm{~W}\end{array}$ & 08 Jan 2006 & 25 & Butterfly Reef & $\begin{array}{l}\text { Southern outer reef. Spur and groove, } \\
\text { medium height }(2-3 \mathrm{ft})\end{array}$ \\
\hline Saba-06-12 & RR09 & $\begin{array}{l}17014.380 \mathrm{~N} \\
63026.915 \mathrm{~W}\end{array}$ & 08 Jan 2006 & 18 & Brain coral reef & $\begin{array}{l}\text { Southern inner reef, rock pavement, } \\
\text { scattered corals }\end{array}$ \\
\hline Saba-06-13 & RR10 & $\begin{array}{l}17033.801 \mathrm{~N} \\
63017.806 \mathrm{~W}\end{array}$ & 09 Jan 2006 & 32 & Fishpot surprise & $\begin{array}{l}\text { sloping pavement with ledges, sand } \\
\text { patches and large rubble, algae, } \\
\text { scattered corals-rotenoned an area with } \\
\text { sand and loose rock at base of rocky } \\
\text { slope with brown algae abundant. }\end{array}$ \\
\hline Saba-06-14 & & $\begin{array}{l}17033.970 \mathrm{~N} \\
63017.227 \mathrm{~W}\end{array}$ & 09 Jan 2006 & 40 & & Fish Trap \\
\hline Saba-06-15 & & $\begin{array}{l}17033.897 \mathrm{~N} \\
63017.730 \mathrm{~W}\end{array}$ & 09 Jan 2006 & 40 & & Fish Trap \\
\hline Saba-06-16 & & $\begin{array}{l}17033.878 \mathrm{~N} \\
63017.168 \mathrm{~W}\end{array}$ & 10 Jan 2006 & 40 & & Fish Trap \\
\hline Saba-06-17 & RR11 & $\begin{array}{l}17033.849 \mathrm{~N} \\
63017.872 \mathrm{~W}\end{array}$ & 10 Jan 2006 & 26 & Lost anchor & $\begin{array}{l}\text { Sloping pavement, patches of large } \\
\text { rubble, algae, scattered corals; red } \\
\text { hind spawning area }\end{array}$ \\
\hline Saba-06-18 & & $\begin{array}{l}17033.837 \mathrm{~N} \\
63017.962 \mathrm{~W}\end{array}$ & 10 Jan 2006 & 30 & & Fish Trap \\
\hline Saba-06-19 & RR12 & $\begin{array}{l}17033.686 \mathrm{~N} \\
63017.629 \mathrm{~W}\end{array}$ & 10 Jan 2006 & 26 & Moonfish Bank & $\begin{array}{l}\text { Sloping pavement, sand patches, } \\
\text { large rubble, algae, scattered corals; } \\
\text { red hind spawning area }\end{array}$ \\
\hline Saba-06-20 & & $\begin{array}{l}17030.751 \mathrm{~N} \\
63013.632 \mathrm{~W}\end{array}$ & 12 Jan 2006 & 29 & Poison Bank & Rotenone \\
\hline Saba-06-21 & & $\begin{array}{l}17028.046 \mathrm{~N} \\
63014.978 \mathrm{~W}\end{array}$ & 12 Jan 2006 & 19 & & Rotenone-northeastern shallow flats \\
\hline Saba-06-22 & & $\begin{array}{l}17026.390 N \\
63027.776 \mathrm{~W}\end{array}$ & 13 Jan 2006 & 26 & & $\begin{array}{l}\text { Rotenone-flat bottom in central area } \\
\text { of bank }\end{array}$ \\
\hline
\end{tabular}




\begin{tabular}{|c|c|c|c|c|c|c|}
\hline Station number & $\begin{array}{l}\text { Roving \& } \\
\text { Rotenone }\end{array}$ & Coordinates & Date & Depth (m) & Local name & Description of habitat \\
\hline Saba-06-23 & & $\begin{array}{l}17030.580 \mathrm{~N} \\
63027.595 \mathrm{~W}\end{array}$ & 13 Jan 2006 & 28 & & $\begin{array}{l}\text { Rotenone-flat bottom in central area } \\
\text { of bank }\end{array}$ \\
\hline Saba-06-24 & & $\begin{array}{l}17020.766 \mathrm{~N} \\
63015.008 \mathrm{~W}\end{array}$ & $14 \operatorname{Jan} 2006$ & 31 & Coral Garden & $\begin{array}{l}\text { Rotenone-coral groove at top of steep } \\
\text { outer dropoff on SE side of Saba Bank, } \\
\text { rock, coral, and sponges, sand at } \\
\text { bottom of groove. }\end{array}$ \\
\hline Saba-06-25 & & $\begin{array}{l}17021.162 \mathrm{~N} \\
63015.138 \mathrm{~W}\end{array}$ & 14 Jan 2006 & 18 & & $\begin{array}{l}\text { Rotenone-near Coral Garden at SE } \\
\text { edge of Saba Bank, small ledge on } \\
\text { edge of sand and rubble flat, } \\
\text { gorgonians, coral and rock }\end{array}$ \\
\hline Saba-06-26 & & & & & & $\begin{array}{l}\text { No label with these-specimens taken } \\
\text { at one or more of the other stations, } \\
\text { but their locality labels were lost }\end{array}$ \\
\hline Saba 2007-01 & & $\begin{array}{l}17.51206 \mathrm{~N} \\
63.2332 \mathrm{~W}\end{array}$ & 20 Jun 2007 & $29-38$ & & $\begin{array}{l}\text { Poison Bank- rotenone-dead and live } \\
\text { coral channel with rubble on deep } \\
\text { reef. }\end{array}$ \\
\hline Saba 2007-02 & & $\begin{array}{l}17.46028 \mathrm{~N} ; \\
63.2517 \mathrm{~W}\end{array}$ & 20 Jun 2007 & 15-19 & & $\begin{array}{l}\text { Twin Peaks- rotenone-algae covering } \\
\text { rock and some sand on a low ridge }\end{array}$ \\
\hline
\end{tabular}

doi:10.1371/journal.pone.0010676.t002

(Mao Tau) in EstimateS v.8.0 software [10]. Total expected richness was estimated using Chao 2 and Jackl estimators.

A Bray-Curtis resemblance matrix was generated from a matrix of presence/absence data from 12 combined roving surveys and rotenone collections in order to produce a non-metric multidimensional scaling (MDS) ordination and group averaged hierarchical clustering dendrogram in PRIMER v. 6.1 software [11]. The ANOSIM statistic was employed to test for a priori differences between habitat types and depth classes. A dendrogram based on group averaged hierarchical cluster techniques was used to illustrate the differences among depth classes (Figure 202). Color codes are derived from the similarity profile (SIMPROF) statistic in Primer 6.1. SIMPROF is less powerful than ANOSIM, intended for a posteriori tests of structure in the data. The test was employed here for representational purposes. A second data matrix of species presence and absence was introduced for an $a$ posteriori test of genuine data structure among Caribbean localities, using the Bray-Curtis resemblance measure to determine the level

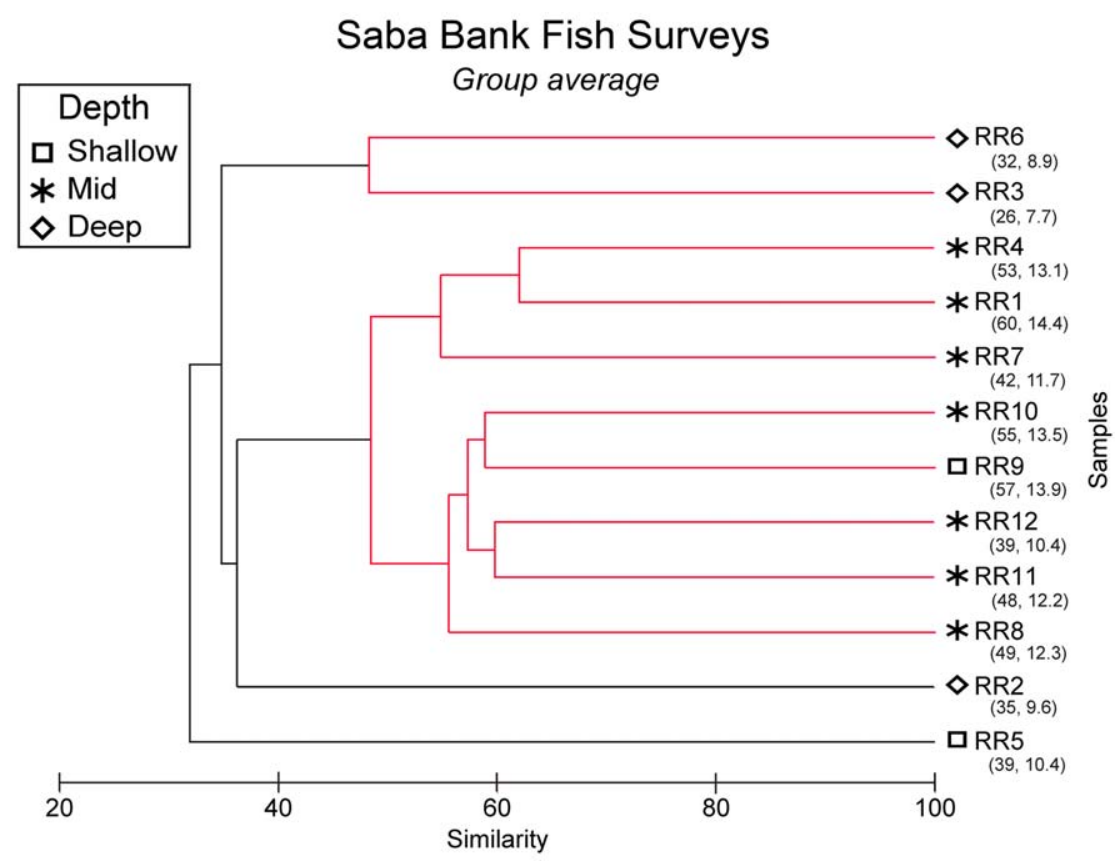

Figure 202. Hierarchical clustering dendrogram of stations showing significant differences (black bars) among fish assemblages at deep stations versus middle-depth and shallow stations (SIMPROF, $P<0.05$ ).

doi:10.1371/journal.pone.0010676.g202 
of similarity among localities. Principal Components Analysis (PCA) was also conducted in PRIMER 6.1 in order to determine the fish species primarily responsible for differences among habitats.

\section{Acknowledgments}

This program was a joint initiative of the Department of Environment and Nature of the Netherlands Antilles and Conservation International. We are especially grateful to the many people who made the biodiversity survey possible, including Andy Caballero of Nature Foundation St. Maarten; David Kooistra, Stanley Peterson, and Jan Den Dulk of Saba Conservation Foundation; Shelley Lundvall, Saba Bank project manager; fishermen Leroy Peterson, Nicky Johnson and Armand Simmons. We thank Jerome Finan, Kris Murphy, and David G. Smith, National Museum of Natural History, Smithsonian Institution, for their help with this project. We thank

\section{References}

1. Meesters EH, Nijkamp H, Bijvoet L (1996) Towards sustainable management of the Saba Bank. Unpublished report to the Department of Public Health and Environment, Curacao, Netherlands Antilles. 42 p.

2. Van der Land J (1977) The Saba Bank, a large atoll in the northeastern Caribbean. FAO Fisheries Reports 200: 469-481.

3. Böhlke JE, Chaplin CGG (1968) Fishes of the Bahamas and adjacent tropical waters. Academy of Natural Sciences of Philadelphia. pp 771.

4. Williams JT (2003) In K.E. Carpenter (ed) 2003. The living marine resources of the Western Central Atlantic. Volume 3: Bony fishes part 2 (Opistognathidae to Molidae, sea turtles and marine mammals. FAO Species Identification Guide for Fishery Purposes and American Society of Ichthyologists and Herpetologists Special Publication No. 5. Rome, FAO. pp 1761-1767.

5. Toller W, Debrot AO, Vermeij MJA, Hoetjes PC (2010) Reef Fishes of Saba Bank, Netherlands Antilles: Assemblage Structure across a Gradient of Habitat Types. In press. This volume of PLoS ONE.

6. Collette BB, Williams JT, Thacker CE, Smith ML (2003) Shore fishes of Navassa Island, West Indies: a case study on the need for rotenone sampling in reef fish biodiversity studies. Aqua J Ichthyol Aquatic Biol 6(3): 89-131.

7. Dennis GD, Smith-Vaniz WF, Golin PL, Hensley DL, McGehee MA (2005) Marine fishes from Islands of the Mona Passage, Greater Antilles. Caribbean Journal of Science 41(4): 716-743.
Menno Van der Velde and Wim Schutten for collecting fishes at several ichthyocide stations. Jill Leger, Yap Films Inc, provided a video clip of fishes filmed at the shipwreck on Saba Bank. Emma L. Hickerson, Flower Garden Banks National Marine Sanctuary (FGBNMS), NOAA, kindly provided information on the number of fish species known to occur at the FGBNMS. Dr. JA Sanchez, University de los Andes, allowed us to use his photograph for Figure 3.

\section{Author Contributions}

Conceived and designed the experiments: MS. Performed the experiments: JTW KEC JLVT PCH WWT PJE MS. Analyzed the data: JTW KEG JLVT PCH PJE. Wrote the paper: JTW. Wrote portions of the paper: KEC PCH PJE. Helped prepare tables and check species identifications: JLVT. Prepared Fig. 1: PCH.

8. Smith-Vaniz WF, Jelks HL, Rocha LA (2006) Relevance of cryptic fishes in biodiversity assessments: A case study at Buck Island Reef National Monument, St. Croix. Bulletin of Marine Science 79(1): 17-48.

9. Smith, CL, Tyler JC, Davis WP, Jones RS, Smith DG, et al. (2003) Fishes of the Pelican Cays, Belize. Atoll Research Bulletin 497: 1-88.

10. Colwell RK (2006) EstimateS: statistical estimation of species richness and shared species from samples.

11. Clarke KR, Warwick RM (2001) Change in Marine Communities: An approach to statistical analysis and interpretation. PRIMER-E: Plymouth, UK.

12. Starck WA, II (1968) A list of fishes of Alligator Reef, Florida with comments on the nature of the Florida reef fish fauna. Undersea Biology 1(1): 5-36.

13. Longley WH, Hildebrand SF (1941) Systematic catalogue of the fishes of Tortugas, Florida, with observations on color, habits, and local distribution. Papers from Tortugas Laboratory No. 34 (Carnegie Institution of Washington Publ. 535). 331 p.

14. Smith-Vaniz WF, Collette BB, Luckhurst B (1999) Fishes of Bermuda: history, zoogeography, annotated checklist, and identification keys. American Society of Ichthyologists and Herpetologists, Special Publication No. 4. 424 p.

15. Clavijo IE, Yntema JA, Ogden JC (1980) An annotated list of the fishes of St. Croix, U.S. Virgin Islands. West Indies Lab, Special Publication ( $2^{\text {nd }}$ ed.). 49 p.

16. Mejia LS, Garzon-Ferreira J, Acero PA (1998) Peces registrados en los complejos arrecifales de los cayos Courtown, Albuquerque, Serrana, y Rancodor, Caribe occidental, Colombia. Boletin Ecotropica: Ecosistemas Tropicales 32: 25-42. 